\title{
Um problema variacional para superfícies de curvatura média constante com bordo livre
}

Márcio Fabiano da Silva

\author{
DISSERTAÇÃO APRESENTADA \\ AO \\ INSTITUTO DE MATEMÁTICA E ESTATÍSTICA \\ DA \\ UNIVERSIDADE DE SÃO PAULO \\ PARA \\ OBTENÇÃO DO GRAU DE MESTRE \\ EM \\ MATEMÁTICA PURA
}

Orientadora: Prof. Rosa Maria dos Santos Barreiro Chaves

Durante a elaboração deste trabalho o autor recebeu auxílio financeiro da FAPESP ( processo número 99/11081-2).

São Paulo, fevereiro de 2002. 


\title{
Um problema variacional para superfícies de curvatura média constante com bordo livre
}

\author{
Este exemplar corresponde à redação \\ final da dissertação devidamente corrigida \\ e defendida por Márcio Fabiano da Silva \\ e aprovada pela comissão julgadora.
}

São Paulo, 28 de fevereiro de 2002.

Banca examinadora:

- Profa. Dra: Rosa Maria dos Santos Barreiro Chaves (orientadora) - IME-USP

- Prof.Dr. Antônio Carlos Asperti - IME-USP

- Prof. Dr. Renato H. L. Pedrosa - IME-UNICAMP 
Dedico esta dissertação à minha amada família: Jane, Sérgio, Jaime, Inês, Vó Nair, Elaine, Márcio e Matheus. Obrigado a vocês todos por caminharem junto a mim na busca de minha realização pessoal e profissional. Agradeço a Deus por me guiar e iluminar nesta dura caminhada. de ce que tu as apprivoisé." 


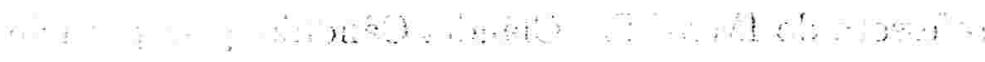

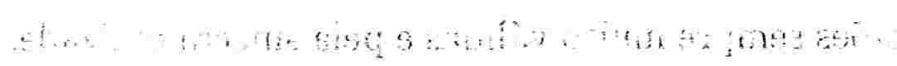

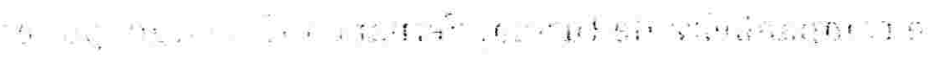

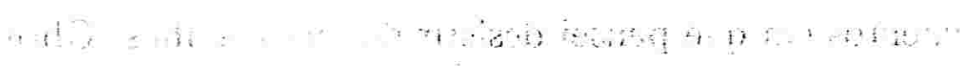

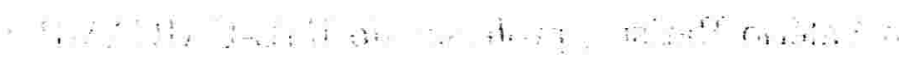

$$
\begin{aligned}
& \therefore \quad \therefore \therefore \quad \therefore \quad \therefore
\end{aligned}
$$




\section{Agradecimentos}

Agradeço à minha orientadora, professora Rosa, pela confiança, amizade, paciência e dedicação. Apesar de estarmos trabalhando juntos já há algum tempo, sinto que tenho ainda muito a aprender com ela. Obrigado Rosa!

Agradeço à professora do IME-USP, Cláudia Cândido, pela participação em meus seminários, pelas sugestões sempre muito válidas e pela sincera amizade. Obrigado Cláudia!

Agradeço à amiga e companheira de turma, Fernanda Camargo, por estar ao meu lado em todos aqueles momentos em que pensei desistir de meus sonhos. Obrigado Fernanda!

Agradeço ao amigo Valério Batista, professor do IME-UNICAMP, pela ajuda com a leitura dos textos em alemão. Obrigado Valério!

Agradeço ao amigo Daniel Tausk, professor do IME-USP, por estar sempre disposto a me ajudar quando precisei. Obrigado Daniel!

Agradeço ao amigo da pós-graduação David Zavaleta pela ajuda na digitação desta dissertação. Obrigado David! 
Agradeço também a todos os meus professores do IME-USP e, em especial, aos professores Aragona, Stavros, Toscano, Roseli, Iole e Francisco Rui, bem como a todos os meus amigos do IME-USP e, em especial, Sandra, Cléber, William, Janaína, Noel, Alexandre, Renata e Ronaldo. Obrigado a todos vocês!

Agradeço também à FAPESP por ter financiado este projeto e pela confiança dada. Obrigado a todos que fazem parte da FAPESP! 


\section{Resumo}

Neste trabalho estudamos o comportamento estável das soluções de um problema isoperimétrico no espaço Euclideano $\mathbb{R}^{3}$. Mais precisamente, caracterizamos a superfície $\mathrm{S}$ que tem menor área dentre todas as superfícies mergulhadas que estão localizadas entre dois planos paralelos $P_{1}$ e $P_{2}$, os encontram ortogonalmente ao longo de seu bordo e encerram um volume $\mathrm{V}$ prescrito. 


\section{Abstract}

In this work we investigate the stability behavior of solutions of an isoperimetric problem in the Euclidean space $\mathbb{R}^{3}$. More precisely, we determine the shape of the surface $\mathrm{S}$ that minimizes the area among all the embedded surfaces which are located between two parallel planes $P_{1}$ and $P_{2}$ and have a prescribed volume $\mathrm{V}$. 


\section{Índice}

Lista de Figuras $\quad$ x

Introdução 1

1 Preliminares $\quad 7$

1.1 Cálculo Variacional . . . . . . . . . . . . . . . . . . . . . 7

1.2 Teoremas de Gauss e Green no Plano . . . . . . . . . . . . . . . . . . . . . 10

1.3 Operadores Elípticos e Princípio do Máximo . . . . . . . . . . . . . . . 11

1.4 Expressões do gradiente, da norma da $2^{\underline{a}}$ forma fundamental em coordenadas e um resultado preliminar para a instabilidade do cilindro . . . . . . 17

2 Caracterizações iniciais das soluções do problema variacional 21

2.1 Reformulação do problema variacional . . . . . . . . . . . . . . . . 21

2.2 As soluções são superfícies de curvatura média constante . . . . . . . . . . 22

2.3 As soluções são superfícies que encontram os planos $P_{1}$ e $P_{2}$ perpendicularmente. . . . . . . . . . . . . . . . . . 25

3 Superfícies rotacionais como soluções do problema variacional $\quad 29$

3.1 Condições para uma superfície ser rotacionalmente simétrica . . . . . . . . 29 
3.2 O Princípio de Reflexão de Alexandrov . . . . . . . . . . . . . . . . . . . 31

4 Superfícies rotacionais em $\mathbb{R}^{3}$ com curvatura média constante $\quad 45$

4.1 Algumas propriedades das superfícies rotacionais em $\mathbb{R}^{3} \ldots \ldots$. . . . . 46

4.2 Caracterização inicial da superfície rotacional S a partir de sua curvatura média $H \ldots \ldots \ldots \ldots$. . . . . . . . . . . . . . . 47

4.3 Determinação de um referencial móvel sobre uma quádrica Q . . . . . . . . 60

4.4 Superfícies de Delaunay . . . . . . . . . . . . . . . . . . . . 61

5 Estabilidade das soluções do problema (P) $\quad 71$

5.1 A segunda variação . . . . . . . . . . . . . . . . . . . . 73

5.2 A instabilidade / estabilidade do cilindro . . . . . . . . . . . . . . 84

5.3 Estabilidade da semiesfera . . . . . . . . . . . . . . . 88

5.4 Instabilidade do ondulóide $\ldots \ldots \ldots \ldots \ldots \ldots$

5.4.1 Instabilidade dos ondulóides para $k \in] 0 ; 0,911[\ldots \ldots 90$

5.4.2 Instabilidade dos ondulóides para $k \in[0,91 ; 1[\ldots \ldots . \ldots 94$

6 Observações finais $\quad 103$

6.1 Comparação das áreas de uma semiesfera e de um cilindro . . . . . . . . 103

6.2 Os mínimos absolutos de (P) são superfícies conexas . . . . . . . . . . . 105 


\section{Lista de Figuras}

1 Representação geral da superfície $\mathrm{S} \ldots \ldots . \ldots 2$

2 Variações $S_{1}$ e $S_{2}$ de $S$ com mesmo volume . . . . . . . . . . . . . 2

3 Variação $S_{3}$ de $S$ com mesmo volume . . . . . . . . . . . . . . 3

1.1 Representação do sistema de coordenadas para S . . . . . . . . . . . 17

2.1 Representação do sistema de coordenadas para $p \in \partial S \ldots \ldots$. . . . . 27

3.1 Imagem da reflexão do ponto $p \in S \bigcap P$ em relação à reta $r_{i} \ldots \ldots$

3.2 Posição da reta $\mathrm{R}$ e do plano $\mathrm{P}$ em relação aos planos $P_{1}$ e $P_{2}$ e à superfície $\mathrm{S} 32$

3.3 Determinação do $1^{\circ}$ ponto de contato $x_{0}$ pela reflexão de um pedaço de $\mathrm{S}$ em relação ao plano $\mathrm{P} \ldots \ldots$. . . . . . . . . . . . . . . . 33

3.4 Representação da possibilidade em que $x_{0} \in \stackrel{0}{S} \ldots \ldots$. . . . . . . . . . 33

3.5 Representação da possibilidade em que $x_{0} \in \partial S \ldots \ldots \ldots$

3.6 Reflexão de $\mathrm{S}$ e $S_{r}$ em relação a $P_{1} \ldots \ldots \ldots \ldots$. . . . . . . . . 34

3.7 Representação da possibilidade em que $x_{0} \in \partial S_{r} \ldots \ldots$. . . . . . . 34

3.8 Outra representação para a possibilidade em que $x_{0} \in \partial S_{r} \ldots \ldots \ldots$

3.9 Representação do conjunto Q . . . . . . . . . . . . . . . 40

3.10 Situação da figura 3.10 após termos mostrado que $S_{r}^{1} \subset S \ldots$. . . . . . . 41

3.11 Situação da figura 3.10 após termos mostrado que $S_{r}^{1} \subset S$ e $S_{r}^{2} \subset S \ldots$. . 42

3.12 Representação de uma situação em que $x_{0}$ é um ponto de contato de $S_{r}^{1}$ e $\mathrm{S} 42$

3.13 Representação de uma situação em que S é desconexa . . . . . . . . . . . 43 
3.14 Representação de uma situação em que $S \bigcap P$ consiste de dois círculos concêntricos . . . . . . . . . . . . . . . . . . . . 44

4.1 Representação do gráfico da função $y=x(t) \ldots \ldots \ldots$. . . . . . . 50

4.2 Representação de um sistema de coordenadas para Q no instante t . . . 60

5.1 Posições dos vetores $\dot{\gamma}(t)=(0,1)$ em relação ao ondulóide . . . . . . . . 72 


\section{Introdução}

" Problema: De todas as curvas simples fechadas no plano com um dado comprimento $\ell$, qual é a que limita a maior área?"

Problemas com formulação análoga ao anterior vêm sendo propostos desde há muito tempo e devido à sua natureza são mais conhecidos como isoperimétricos. Conforme consta em [8], existem evidências de que os gregos já sabiam que a circunferência era uma solução do problema apresentado inicialmente, mas uma prova satisfatória deste fato foi primeiramente dada por Weierstrass somente em 1870, quando ele utilizou resultados do Cálculo Variacional para garantir a existência de soluções de um dado problema isoperimétrico. Uma discussão mais detalhada do problema acima é encontrada em [8] e [19]. Um breve histórico da motivação para o problema que estaremos considerando nesta dissertação pode ser encontrado na página 340 da referência [4].

Nesta dissertação nos propomos a estudar, no espaço euclideano $\mathbb{R}^{3}$,a forma das superfícies que têm menor área dentre todas as superfícies mergulhadas que estão localizadas entre dois planos paralelos $P_{1}$ e $P_{2}$, encontram-nos ortogonalmente ao longo de seu bordo e englobam um volume $\mathrm{V}$ prescrito.

Este resultado foi resolvido independentemente por Maria Athanassenas em [3] e [2] e por Tomas Vogel em [22]. Ambos os autores utilizam as técnicas básicas abordadas em [4] onde foi estabelecida a definição de estabilidade para hipersuperfícies com curvatura média constante, no espaço euclideano $\mathbb{R}^{n+1}$. O trabalho de Vogel trata o problema sob um ponto de vista físico, considerando uma gota líquida, prensada entre dois planos paralelos $P_{1}$ e $P_{2}$, sob ausência de gravidade. No entanto, em seus trabalhos [3] e [2] Maria Athanassenas 
trata-o pelo ponto de vista matemático, considerando $\mathrm{S}$ uma superfície mergulhada em $\mathbb{R}^{3}$ de classe $C^{2}$, orientável, que está entre os planos paralelos $P_{1}$ e $P_{2}$ e além disto é suposta compacta e conexa com bordo $\partial S$ contido em $P_{1} \cup P_{2}$ e $(\mathrm{P})$ o seguinte problema variacional (ou isoperimétrico):

"Minimizar o funcional área $\mathrm{A}(\mathrm{S})$ sob a condição adicional que o volume $\mathrm{V}$ determinado por S é constante" ( ver Figuras 1, 2 e 3).

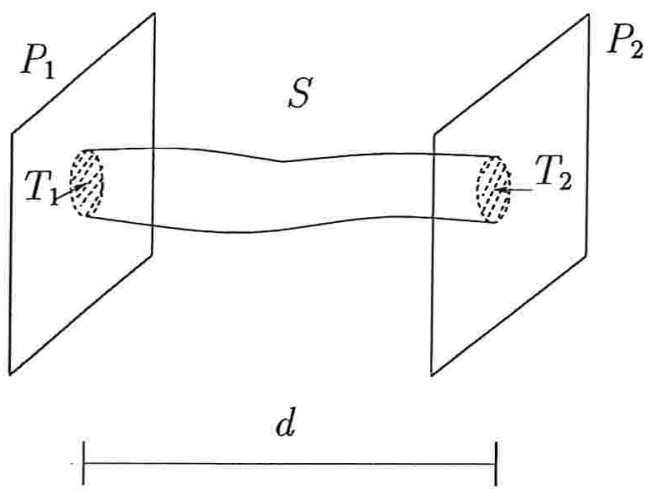

Figura 1: Representação geral da superfície S

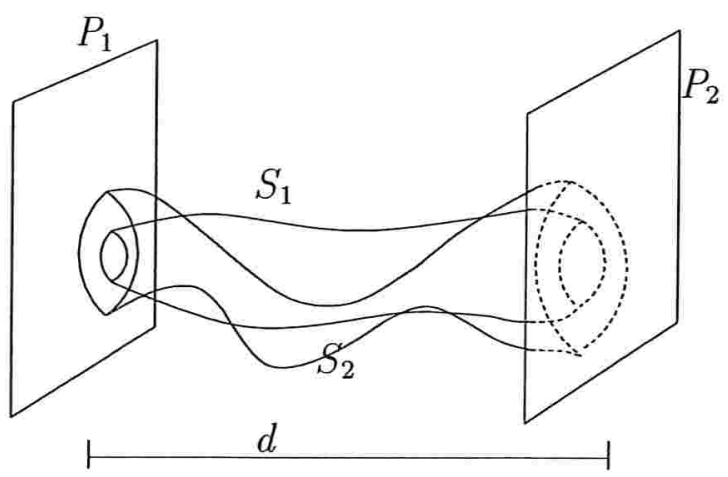

Figura 2: Variações $S_{1}$ e $S_{2}$ de $\mathrm{S}$ com mesmo volume

Observamos que faz sentido falar no volume $V(S)$, pois sob as hipóteses dadas anteriormente, existe um sólido compacto $\mathrm{K}$ de volume $V(S)=V$ cuja fronteira compõe-se da união de $\mathrm{S}$ com as "tampas" $T_{1}, T_{2}$ formadas pelas regiões dos planos $P_{1}$ e $P_{2}$ delimitadas pelas curvas que são o bordo $\partial S$ de $\mathrm{S}$.

Comparando os artigos [22] de Vogel e [3] de M.Athanassenas, podemos notar que a. 


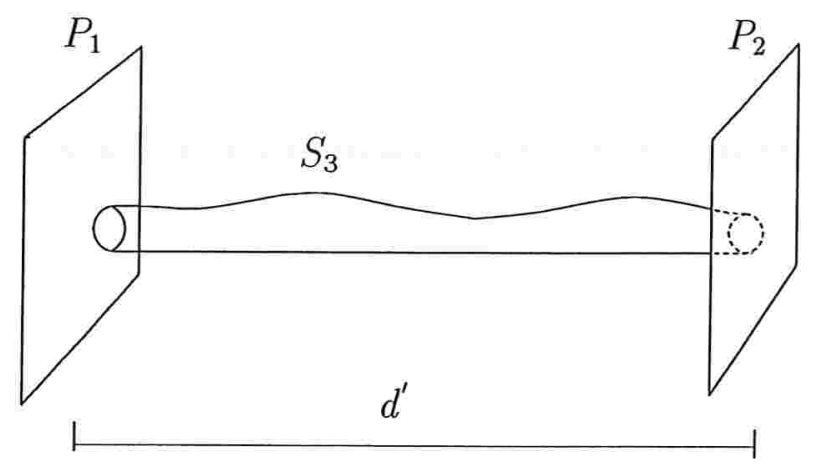

Figura 3: Variação $S_{3}$ de $\mathrm{S}$ com mesmo volume

metodologia ali empregada por Athanassenas na apresentação dos enunciados, bem como de suas provas, está bem mais próxima da referência [4], que constitui a base para os trabalhos de ambos os autores. Em razão disto, escolhemos a versão adotada por M. Athanassenas para detalharmos os resultados que resolvem o problema $(\mathrm{P})$ anteriormente descrito. Observamos ainda que há um cuidado a ser tomado quando tentamos utilizar os resultados de [4], pois lá o bordo está fixo, enquanto que no nosso caso temos bordo livre.

A dissertação está dividida em 6 capítulos, sendo que no capítulo 1 apresentamos os resultados preliminares que serão necessários para os capítulos seguintes. Estes resultados foram organizados pela ordem em que serão utilizados.

No capítulo 2, supomos que uma tal superfície S seja solução do problema variacional (P) e buscamos condições que devem ser satisfeitas por S. Com base em resultados, principalmente do Cálculo de Variações e Cálculo Diferencial em $\mathbb{R}^{n}$, mostramos através dos Teoremas 2.2.1 e 2.3.1 que $\mathrm{S}$ deve ter curvatura média $\mathrm{H}$ constante e deve encontrar os planos $P_{1}$ e $P_{2}$ perpendicularmente ao longo de seu bordo $\partial S$.

Em seguida, no capítulo 3, descrevemos o Princípio de Reflexão de Alexandrov e procedemos como em [1] para provar que se "S é uma superfície mergulhada em $\mathbb{R}^{3}$, de classe $C^{2}$,orientável, que está entre os planos paralelos $P_{1}$ e $P_{2}$, conexa e compacta com bordo $\partial S$ contido em $P_{1} \bigcup P_{2}$ e ainda com curvatura média $\mathrm{H}$ constante, então S é rotacionalmente simétrica ao redor de um eixo perpendicular a $P_{1}, P_{2}$ " (Teorema 3.2.1).

Com isto, temos que as soluções do problema (P) satisfazem todas as hipóteses do Teorema 3.2.1, e portanto segue o resultado acima também para elas. Na demonstração deste teorema são usados ainda o Princípio do Máximo e o Lema de Hopf para um ponto 
de bordo.

Com o que foi mostrado até então, as soluções do problema apresentado inicialmente são superfícies rotacionalmente simétricas em $\mathbb{R}^{3}$ de curvatura média $\mathrm{H}$ constante, que foram caracterizadas por Delaunay em seu trabalho [11] como sendo superfícies rotacionais geradas por curvas descritas pela trajetória do foco de seções cônicas que rolam, sem deslizar, ao longo de um eixo. Além disto, estas superfícies compreendem exatamente o cilindro, o catenóide, o nodóide, o ondulóide, bem como a esfera e o plano. Esta. classificação é tratada no capítulo 4 da dissertação através dos Teoremas 4.2.2 e 4.4.1. Ainda cabe observarmos que, devido às condições do problema, interessa-nos somente a esfera (respectivamente a semiesfera, se colocarmos como hipótese o bordo estar somente . em um dos planos $P_{i}$ ), o cilindro e o ondulóide. As demais superfícies são descartadas, visto que o plano não delimita um sólido, o catenóide nunca encontra os planos $P_{1}$ e $P_{2}$ perpendicularmente e o nodóide tem auto-intersecções.

Dedicamos o capítulo 5 ao estudo da estabilidade das superfícies. Da Proposição 5.1.2 segue que uma superfície $\mathrm{S}$ tem curvatura média $H_{0}$ e encontra os planos $P_{i}$ perpendicularmente ao longo de seu bordo se e somente se ela for ponto crítico do funcional área, para variações que preservam o volume. Isto mostra que o cilindro, a esfera ( ou semiesfera ) e o ondulóide são, de fato, as únicas possíveis soluções do problema (P). Pela Proposição 5.1 .2 vemos também que encontrar os pontos críticos do funcional área, com a condição do volume permanecer constante, equivale a resolver o problema do método dos multiplicadores de Lagrange associado.

Como estamos interessados em obter área mínima com a condição da variação fixar volume, então a definição de estabilidade dada no capítulo 5 ( ver Definição 5.1.4 ) esclarece nosso particular interesse na estabilidade das superfícies.

Com base no artigo [4], provamos com a Proposição 5.1.3 e Lema 5.1.6 que a condição de estabilidade é equivalente a mostrar que $\int_{S}\left\{\|\nabla f\|^{2}-\|B\|^{2} f^{2}\right\} d a \geq 0$, para toda variação rotacionalmente simétrica da superfície cuja componente normal $\mathrm{f}$ satisfaz a condição adicional $\int_{S} f . d a=0$ (condição de valor médio nulo ), onde da denota o elemento de superfície, $\nabla$ é o gradiente e $\|B\|$ é a norma da $2^{\underline{a}}$ forma fundamental de $\mathrm{S}$.

Para concluir este capítulo 5, estudamos o comportamento das soluções do problema (P) no que se refere à estabilidade. No Teorema 5.2.1 mostramos que o cilindro de raio $\rho$ 
é estável somente no caso $\rho \pi \geq \operatorname{dist}\left(P_{1}, P_{2}\right)$. Isto é feito através de resultados do Cálculo Variacional. O Teorema 5.3.1 nos garante a estabilidade da semiesfera a partir da estabilidade da esfera, provada em [4]. Para o ondulóide encontramos uma função teste adequada tal que $\int_{S} f . d a=0$ e $\int_{S}\left\{\|\nabla f\|^{2}-\|B\|^{2} f^{2}\right\} d a<0$, de onde segue que o ondulóide é instável, eliminando-o portanto como solução do nosso problema (Teoremas 5.4.1 e 5.4.2). Existe uma dificuldade ao se provar este resultado, pois de acordo com a definição de estabilidade tomada, bem como pelo procedimento adotado, não foi possível encontrar uma única função teste $\mathrm{f}$ que garantisse de imediato a instabilidade desta superfície.

O capítulo 6 está reservado para as conclusões finais. Neste momento, apresentamos em que caso o cilindro tem área superficial menor que a da semiesfera e vice-versa, dependendo da relação entre o volume $\mathrm{V}$ encerrado e a distância d entre os planos $P_{1}, P_{2}$. Finalmente, mostramos que uma superfície $\mathrm{S}$ desconexa não pode ocorrer como mínimo absoluto do problema variacional $(\mathrm{P})$, de onde segue que $\mathrm{S}$ só pode ser conexa. Para isto, procedemos por indução no número de componentes conexas de S.

Para uma leitura básica e complementar referente ao assunto em questão, recomendamos as seguintes referências: [6],[7], [9], [10], [12], [14], [15], [16], [17], [20] e [21]. 


\section{Preliminares}

Neste capítulo, tratamos dos resultados básicos e necessários aos capítulos posteriores.

\subsection{Cálculo Variacional}

Conforme já observamos, o Cálculo Variacional desempenha um papel fundamental em nosso trabalho quando buscamos condições necessárias que devem ser satisfeitas por uma solução do problema variacional (P) considerado. Trataremos deste assunto por toda a dissertação e, em especial, no capítulo 2. Em razão disto, definimos a seguir alguns conceitos básicos do Cálculo de Variações e apresentamos os resultados deste tópico que utilizaremos mais adiante.

Funcional: entendemos por um funcional uma quantidade ou função que por sua vez depende de uma ou mais funções. Como exemplo temos o comprimento de arco $\ell[\gamma(t)]$ de uma curva regular dada por $\gamma(t)=(t, y(t)) \in C^{1}$, onde $t \in\left[t_{0}, t_{1}\right]$. Sabemos que

$$
\ell[\gamma(t)]=\int_{t_{0}}^{t_{1}} \sqrt{1+\left(\frac{d}{d t} y(t)\right)^{2}} d t
$$

e portanto, $\ell[\gamma(t)]$ depende da função $y(t) \in C^{1}$, que pode ser tomada arbitrariamente.

Podemos encontrar na vasta literatura de Cálculo Variacional uma definição geral de variação; no entanto, descrevemos aqui uma versão que mais se aproxima das nossas condições: 
Definição 1.1.1. Seja $\gamma(t)=(x(t), t), x(t)>0$, uma curva parametrizada regular de classe $C^{2}\left([0, d], \mathbb{R}^{2}\right)$, onde $d=\operatorname{dist}\left(P_{1}, P_{2}\right)$, onde $P_{1}$ e $P_{2}$ são os planos descritos no problema $(P)$. Uma variação de $\gamma$ é uma aplicação

$\left.\gamma_{\epsilon}:[0, d] \longrightarrow \mathbb{R}^{2} \in C^{2}\left([0, d], \mathbb{R}^{2}\right), \epsilon \in\right]-\epsilon_{0}, \epsilon_{0}[$, tal que:

(i) $\gamma(t, 0)=: \gamma_{0}(t)=\gamma(t), \forall t \in[0, d]$;

(ii) para cada $\epsilon \in]-\epsilon_{0}, \epsilon_{0}\left[, \gamma_{\epsilon}(t)\right.$ é diferenciável com relação a $\epsilon$.

Devido à expressão de $\gamma_{\epsilon}(t)=\left(x_{\epsilon}(t), t\right)$, tomamos a variação da curva $\gamma$ como sendo dada por $x_{\epsilon}(t)$, com $x_{0}(t)=x(t), \forall t \in[0, d]$ (isto segue da condição (i) anterior). Denotamos por $\Psi=\left.\frac{d}{d \epsilon} x_{\epsilon}(t)\right|_{\epsilon=0}$ o vetor variação de $x_{\epsilon}(t)$.

Devido à expressão de $\gamma(t)=(x(t), t)$, mostraremos no capítulo 5 que a área $A(S)$ e o volume $V(S)$ da superfície $S$ são dados em função somente de $x(t)$ e portanto, podemos denotá-los por $A(x)$ e $V(x)$. Em razão disto, indicaremos por $A(\epsilon)=A\left(x_{\epsilon}\right)$ e $V(\epsilon)=$ $V\left(x_{\epsilon}\right)$ a área e o volume, respectivamente, de uma variação $\varphi_{\epsilon}$, determinada por $x_{\epsilon}$, da superfície S. Com estas notações, dizemos que uma variação $x_{\epsilon}$ preserva volume se $\left.V(\epsilon)=V(0)=V\left(x_{0}\right), \forall \epsilon \in\right]-\epsilon_{0}, \epsilon_{0}[$.

Além disto, a componente normal do vetor variação $\Psi=\left.\frac{d}{d \epsilon} x_{\epsilon}(t)\right|_{\epsilon=0}$ é dada por:

$$
f=<\left.\frac{d}{d \epsilon} \varphi_{\epsilon}(t, \phi)\right|_{\epsilon=0}, N(t, \phi)>
$$

onde $N(t, \phi)$ é o campo normal unitário de $\mathrm{S}$ e $<,>$ é o produto interno em $\mathbb{R}^{3}$.

Finalmente, observamos que se a superfície S é dada localmente como gráfico de uma função $u \in C^{2}$ ( como no capítulo 2 ) então procedemos de forma análoga ao que foi feito anteriormente para considerarmos variações $u_{\epsilon}$ de $\mathrm{S}$.

Conforme apontamos na introdução desta dissertação, a existência de uma solução de um problema variacional, em particular para o problema (P) considerado, é garantida pelo teorema de Weierstrass: "Se $f: K \subset \mathbb{R}^{n} \longrightarrow \mathbb{R}$ é contínua e $\mathrm{K}$ é um compacto nãovazio então f assume máximo e mínimo em K". A prova deste fato pode ser encontrada na grande maioria de livros de Cálculo ou Análise e será omitida aqui. Reservamo-nos a discutir outros resultados importantes de Cálculo Variacional, que utilizaremos no decorrer deste trabalho. Começamos com o:

Lema 1.1.2. (Lema fundamental do Cálculo de Variações) Seja $\Omega \subseteq \mathbb{R}^{n}$ um aberto. Se 
para cada função contínua $\eta(x), x \in \bar{\Omega}$, que se anula no bordo de $\partial \Omega$ vale

$$
\int_{\bar{\Omega}} \eta(x) \cdot \Phi(x) d x=0
$$

$\operatorname{com} \Phi(x)$ uma função contínua de $x \in \bar{\Omega}$, então $\Phi(x)=0, \forall x \in \bar{\Omega}$.

Prova: Suponha, por absurdo, que existe $x=\bar{x} \in \Omega$ tal que $\Phi(x) \neq 0$. Pela continuidade de $\Phi$, podemos tomar a vizinhança $\mathrm{V}$ de $x=\bar{x} \in \Omega$ dada pela componente conexa que contém $x=\bar{x}$ do conjunto $\{x \in \Omega / \Phi(x) \neq 0\}$, ou seja, $\Phi$ conserva seu sinal em V. Mas então, tomando a função $\eta(x)$ definida em $\bar{\Omega}$ por:

$$
\eta(x)=\left\{\begin{aligned}
\Phi(x), & \text { se } x \in V \\
0, & \text { se } x \in \bar{\Omega}-V
\end{aligned}\right.
$$

que é contínua e se anula no bordo $\partial \Omega$, obtemos que (1.2) se torna:

$$
\int_{\bar{\Omega}} \eta(x) \cdot \Phi(x) d x=\int_{V} \eta(x) \cdot \Phi(x) d x+\int_{\bar{\Omega}-V} \eta(x) \cdot \Phi(x) d x=\int_{V} \Phi^{2}(x) d x>0,
$$

o que contraria a hipótese de que (1.2) é igual a zero.

Portanto, $\Phi \equiv 0$ em $\Omega$. Como $\Phi(x)$ é contínua, $\forall x \in \bar{\Omega}$, segue que $\Phi \equiv 0$ em $\bar{\Omega}$.

Observação 1.1.3. O lema e sua demonstração não mudam se exigirmos que $\eta \in C^{k}$ ou se suprimirmos a hipótese de que $\eta(x)$ se anula no bordo $\partial \Omega$. Além disto, como impomos que $\eta(x)=0$ se $x \in \partial \Omega$, então

$$
\int_{\partial \Omega} \eta(x) \Phi(x) d x=0
$$

e portanto, (1.2) equivale a $\int_{\Omega} \eta(x) \Phi(x) d x=0$.

Quando procuramos pelos pontos críticos do funcional área $A(S)$, supondo o volume $V(S)=V$ constante, já comentamos que um procedimento equivalente é resolver o problema dos multiplicadores de Lagrange, cuja existência é provada a seguir.

Lema 1.1.4. (O método dos multiplicadores de Lagrange) Seja $c \in \mathbb{R}$ um valor regular de $w=g(x, y, z)$ e $S$ a superfície de nível de ordem $c$ de $g$. Seja também $f: A \subset \mathbb{R}^{3} \longrightarrow \mathbb{R}$, $A$ aberto, $S \subset A$. Se $\left(x_{0}, y_{0}, z_{0}\right)$ é máximo local ou mínimo local de $f$ restrita a $S$, então $\exists \lambda \in \mathbb{R}$ tal que

$$
\nabla f\left(x_{0}, y_{0}, z_{0}\right)=\lambda \nabla g\left(x_{0}, y_{0}, z_{0}\right)
$$


Prova: Suponhamos que $\left(x_{0}, y_{0}, z_{0}\right)$ seja um máximo local de f. Então, $\exists \epsilon>0$ tal que $f(x, y, z) \leq f\left(x_{0}, y_{0}, z_{0}\right), \forall(x, y, z) \in B_{\epsilon}\left(x_{0}, y_{0}, z_{0}\right) \bigcap S$.

Como $\nabla g\left(x_{0}, y_{0}, z_{0}\right) \neq(0,0,0)$, suponhamos $\frac{\partial g}{\partial z}\left(x_{0}, y_{0}, z_{0}\right) \neq 0$. Pelo teorema das funções implícitas, existem abertos $\mathrm{U}, \mathrm{V},\left(x_{0}, y_{0}\right) \in U, z_{0} \in V, U \times V \subset B_{\epsilon}\left(x_{0}, y_{0}, z_{0}\right)$, $h: U \longrightarrow V \in C^{1}$ tal que $g(x, y, h(x, y))=c, \forall(x, y) \in U$ e $h\left(x_{0}, y_{0}\right)=z_{0}$.

Como $f(x, y, h(x, y)) \leq f\left(x_{0}, y_{0}, z_{0}\right), \forall(x, y) \in U$, visto que $U \times V \subset B_{\epsilon}\left(x_{0}, y_{0}, z_{0}\right)$, então $\left(x_{0}, y_{0}\right)$ é máximo local de $F: U \longrightarrow \mathbb{R}$ dada por:

$$
F(x, y)=f(x, y, h(x, y))
$$

Portanto

$$
\begin{aligned}
& 0=\frac{\partial F}{\partial x}\left(x_{0}, y_{0}\right)=\nabla f\left(x_{0}, y_{0}, z_{0}\right) \cdot\left(1,0, \frac{\partial h}{\partial x}\left(x_{0}, y_{0}\right)\right) \\
& 0=\frac{\partial F}{\partial y}\left(x_{0}, y_{0}\right)=\nabla f\left(x_{0}, y_{0}, z_{0}\right) \cdot\left(0,1, \frac{\partial h}{\partial y}\left(x_{0}, y_{0}\right)\right) .
\end{aligned}
$$

Como $\left(1,0, \frac{\partial h}{\partial x}\left(x_{0}, y_{0}\right)\right)$ e $\left(0,1, \frac{\partial h}{\partial y}\left(x_{0,}, y_{0}\right)\right.$ são linearmente independentes, tangentes a S e perpendiculares a $\nabla f\left(x_{0}, y_{0}, z_{0}\right)$, e $\nabla g\left(x_{0}, y_{0}, z_{0}\right)$ é perpendicular a $\mathrm{S}$, então segue (1.3).

No nosso caso, em que S será dada localmente como gráfico de uma função (como no capítulo 2), consideraremos $c=0, \mathrm{f}$ e g como sendo as funções área e volume de $\mathrm{S}$.

\subsection{Teoremas de Gauss e Green no Plano}

Para a prova do Teorema 2.2.1 usaremos a seguinte versão do teorema da divergência de Gauss no plano.

Teorema 1.2.1. Seja $\Omega \subseteq \mathbb{R}^{2}$ um aberto limitado com $\partial \Omega \in C^{1}$ e seja $\nu$ a normal unitária exterior a $\partial \Omega$. Para qualquer campo vetorial $w \in C^{0}(\bar{\Omega}) \bigcap C^{1}(\Omega)$, temos:

$$
\int_{\Omega} d i v w d x=\int_{\partial \Omega} w \cdot \nu d s
$$

onde ds denota o elemento de área unidimensional em $\partial \Omega$. 
Este é um resultado clássico dos cursos de Cálculo e omitiremos sua prova aqui. No entanto, vamos obter a seguir a chamada primeira identidade de Green.

Corolário 1.2.2. Sejam $u, v \in C^{0}(\bar{\Omega}) \bigcap C^{1}(\Omega)$, onde $\Omega \subseteq \mathbb{R}^{2}$ é um aberto limitado com $\partial \Omega \in C^{1}$. Então:

$$
\int_{\Omega} \frac{\nabla u \cdot \nabla v}{\sqrt{1+\|\nabla u\|^{2}}} d x+\int_{\Omega} v \operatorname{div}\left(\frac{\nabla u}{\sqrt{1+\|\nabla u\|^{2}}}\right) d x=\int_{\partial \Omega} v \frac{\nabla u \cdot \nu}{\sqrt{1+\|\nabla u\|^{2}}} d s,
$$

onde $\nu$ e ds foram definidas no Teorema 1.2.1.

Prova: Temos que

$$
v \operatorname{div} w=\sum_{j=1}^{2} v \frac{\partial w_{j}}{\partial x_{j}}=\sum_{j=1}^{2}\left[\frac{\partial}{\partial x_{j}}\left(v w_{j}\right)-\frac{\partial v}{\partial x_{j}} w_{j}\right]=\operatorname{div}(v w)-\nabla v \cdot w .
$$

Em particular, se $w=\frac{\nabla u}{\sqrt{1+\|\nabla u\|^{2}}}$, segue a fórmula:

$$
v \operatorname{div}\left(\frac{\nabla u}{\sqrt{1+\|\nabla u\|^{2}}}\right)=\operatorname{div}\left(v \frac{\nabla u}{\sqrt{1+\|\nabla u\|^{2}}}\right)-\frac{\nabla u \cdot \nabla v}{\sqrt{1+\|\nabla u\|^{2}}} .
$$

Integrando (1.6) em $\Omega$, temos

$$
\int_{\Omega} \operatorname{div}\left(v \frac{\nabla u}{\sqrt{1+\|\nabla u\|^{2}}}\right) d x=\int_{\Omega} \frac{\nabla u \cdot \nabla v}{\sqrt{1+\|\nabla u\|^{2}}} d x+\int_{\Omega} v \operatorname{div}\left(\frac{\nabla u}{\sqrt{1+\|\nabla u\|^{2}}}\right) d x .
$$

Além disto, (1.4) é dada por $\int_{\Omega} \operatorname{div}\left(v \frac{\nabla u}{\sqrt{1+\|\nabla u\|^{2}}}\right) d x=\int_{\partial \Omega} v \frac{\nabla u \cdot \nu}{\sqrt{1+\|\nabla u\|^{2}}} d s$.

Das duas últimas igualdades segue a identidade de Green.

\subsection{Operadores Elípticos e Princípio do Máximo}

Destacamos agora alguns resultados que serão utilizados no capítulo 3.

Lema 1.3.1. Sejam $K \subset \mathbb{R}^{n}$ compacto, Jordan-mensurável (i.e, $m(\partial K)=0$ ), $X$ um espaço topológico e $f: K \times X \longrightarrow \mathbb{R}$ uma função contínua. Se $\Phi: X \longrightarrow \mathbb{R}$ é dada por $\Phi(x)=\int_{K} f(t, x) d t$ então $\Phi$ é contínua em $x$. 
Prova: Como $f: K \times X \longrightarrow \mathbb{R}$ é contínua e K é compacto, então f é uniformemente contínua com respeito à variável t. Ou seja, dados $x_{0} \in X$ e $\epsilon>0, \exists V \subset X$ vizinhança de $x_{0}$ tal que $\forall t \in K, \forall x \in V,\left|f(t, x)-f\left(t, x_{0}\right)\right|<\epsilon$.

Portanto, $\left|\Phi(x)-\Phi\left(x_{0}\right)\right| \leq \int_{K}\left|f(t, x)-f\left(t, x_{0}\right)\right| d t<\epsilon \cdot m(K)<+\infty$, pois $\mathrm{K}$ é compacto e $m(\partial K)=0$.

Então, tomando $\tilde{\epsilon}:=\epsilon \cdot m(K)$ temos que dado $\tilde{\epsilon}>0, \exists \delta>0$ tal que $\left|\Phi(x)-\Phi\left(x_{0}\right)\right|<\tilde{\epsilon}$ ( na verdade, para qualquer $\delta>0$ a desigualdade é satisfeita).

Como o próximo resultado é clássico, omitiremos sua prova

Lema 1.3.2. (Máximos e Mínimos de formas quadráticas) Seja $A: \mathbb{R}^{n} \longrightarrow \mathbb{R}^{n}$ uma matriz simétrica. Então,

$$
\left\{\begin{array}{l}
\min _{\|\xi\|=1}\langle A \xi, \xi\rangle=\text { menor autovalor de } A:=\lambda \\
\max _{\|\xi\|=1}\langle A \xi, \xi\rangle=\text { maior autovalor de } A:=\Lambda
\end{array}\right.
$$

Ou seja, $\lambda \leq<A \xi, \xi>\leq \Lambda, \forall \xi \in \mathbb{R}^{n},\|\xi\|=1$. Se $\xi \in \mathbb{R}^{n}-\{0\}$ é qualquer, então:

$$
\lambda\|\xi\|^{2} \leq<A \xi, \xi>\leq \Lambda\|\xi\|^{2} \text {, onde }<A \xi, \xi>=\sum_{i, j=1}^{n} a_{i j} \xi_{i} \xi_{j}
$$

onde $A=\left(a_{i j}\right)_{i, j=1, \ldots, n}$.

Agora, vamos formalizar alguns conceitos básicos da teoria de Equações Diferenciais Parciais, que serão utilizados no capítulo 3 quando tratarmos da equação de curvatura média constante.

Definição 1.3.3. Seja $\mathcal{L}$ um operador diferencial de $2^{a}$ ordem da forma

$$
\mathcal{L} v:=\sum_{i, j=1}^{2} a_{i j}(x) v_{i j}(x)+\sum_{i=1}^{2} b_{i}(x) v_{i}(x)
$$

$\operatorname{com} a_{i j}=a_{j i}$, para $i, j=1,2, \quad v_{i j}=\frac{\partial^{2} v}{\partial x_{i} \partial x_{j}}, \quad v_{i}=\frac{\partial v}{\partial x_{i}}, x \in \Omega \subset \mathbb{R}^{2} e v \in C^{2}(\Omega)$.

Dizemos que $\mathcal{L}$ é um operador elíptico em $\Omega \subset \mathbb{R}^{2}$ se a matriz coeficiente $\left[a_{i j}(x)\right]_{i, j=1,2}$ é definida positiva, $\forall x \in \Omega$, ou seja, os menores principais de $A=\left[a_{i j}(x)\right]_{i, j=1,2}$, definidos 
por

$k=1,2$, são todos estritamente positivos.

$$
\operatorname{det} A^{(k)}=\operatorname{det}\left[\begin{array}{lll}
a_{11} & \cdots & a_{1 k} \\
\vdots & \vdots & \vdots \\
a_{k 1} & \cdots & a_{k k}
\end{array}\right]
$$

Associada ao operador $\mathcal{L}$ temos a equação diferencial parcial $\mathcal{L} v=0$. Esta equação é dita elíptica se o operador $\mathcal{L}$ for elíptico, independentemente da solução $v$.

Observação 1.3.4. Se o operador $\mathcal{L}$, como em (1.8), for elíptico então o menor e o maior autovalor $\lambda(x)$ e $\Lambda(x)$ respectivamente, da matriz dos $\left[a_{i j}(x)\right]_{i, j=1,2}$, dados por

$$
\begin{aligned}
& \lambda(x)=\frac{a_{11}(x)+a_{22}(x)-\sqrt{\left(a_{11}(x)-a_{22}(x)\right)^{2}+4\left(a_{12}(x)\right)^{2}}}{2} \\
& \Lambda(x)=\frac{a_{11}(x)+a_{22}(x)+\sqrt{\left(a_{11}(x)-a_{22}(x)\right)^{2}+4\left(a_{12}(x)\right)^{2}}}{2}
\end{aligned}
$$

são estritamente positivos, visto que $a_{11}(x), a_{22}(x)$ e $a_{11}(x) a_{22}(x)-\left(a_{12}(x)\right)^{2}$ os são.

Consequentemente, se o operador simétrico $\mathcal{L}$ for elíptico então pelo Lema 1.3.2 temos a desigualdade:

$$
0<\lambda(x)\|\xi\|^{2} \leq \sum_{i, j=1}^{2} a_{i j} \xi_{i} \xi_{j} \leq \Lambda(x)\|\xi\|^{2}, \quad \forall \xi=\left(\xi_{1}, \xi_{2}\right) \in \mathbb{R}^{2}-\{0\}, x \in \Omega .
$$

Definição 1.3.5. Dizemos ainda que o operador $\mathcal{L}$, como em (1.8), é uniformemente elíptico em $\Omega \subset \mathbb{R}^{2}$ se:

(i) vale a desigualdade (1.11), $\forall x \in \Omega$;

(ii) $\frac{\Lambda(x)}{\lambda(x)}$ é limitado em $\Omega, \quad \forall x \in \Omega$.

Observação 1.3.6. Ressalvamos que se o operador $\mathcal{L}$ for elíptico em $\Omega$ e os coeficientes $a_{i j}(x), i, j=1,2$, da matriz dos $\left[a_{i j}(x)\right]_{i, j=1,2}$ forem contínuos em $x, \forall x \in \Omega$, então seguirá imediatamente que $\mathcal{L}$ é uniformemente elíptico em qualquer domínio compacto $\Omega^{*}$ contido em $\Omega$. De fato,

- $\mathcal{L}$ é elíptico em $\Omega \Longrightarrow$ vale (1.11), $\forall x \in \Omega$; em particular, isto é válido em $\Omega^{*}$.

- $a_{i j}(x)$ são contínuos em $\Omega^{*} \subset \Omega \Longrightarrow \lambda(x)$ e $\Lambda(x)$ são contínuos em $x, \forall x \in \Omega^{*}$, devido às suas expressões $\Longrightarrow \frac{\Lambda(x)}{\lambda(x)}$ é contínuo em $x, \forall x \in \Omega^{*}$, pois $\lambda(x) \neq 0, \forall x \in$ $\Omega^{*} \Longrightarrow \frac{\Lambda(x)}{\lambda(x)}$ é limitado em $\Omega^{*}$, visto que $\Omega^{*}$ é compacto. 
Estamos agora em condições de apresentar o Princípio do Máximo Forte e o Lema de Hopf, que são os dois principais resultados preliminares utilizados no capítulo 3 da dissertação. Reproduzimos a seguir as provas destes resultados com base nas referências [5] e [13]. Antes disto, porém, enunciamos outros dois resultados preliminares, que são utilizados na demonstração do Lema de Hopf, e cujas provas podem ser encontradas nas mesmas referências citadas há pouco.

Proposição 1.3.7. ( O Princípio do Máximo Clássico) Seja $\mathcal{L}$, como em (1.8), um operador elíptico em um domínio limitado $\Omega$ de $\mathbb{R}^{n}$. Suponha $\mathcal{L} u>0$ em $\Omega$ para uma função $u \in C^{2}(\Omega)$. Então, a função u não pode ter um máximo local no interior de $\Omega$.

Proposição 1.3.8. Seja $\mathcal{L}$, como em (1.8), um operador elíptico em um domínio $\Omega$ e para o qual ainda vale que $\frac{\left|b_{i}\right|}{\lambda}$ é limitado em $\Omega$, para cada $i$ ( $\lambda$ é o menor autovalor da matriz dos $\left.\left(a_{i j}\right)\right)$. Se $\mathcal{L} u \geq 0$ em $\Omega$ para uma função $u \in C^{2}(\Omega)$, então u não pode ter um máximo local em $\Omega$, a menos que u seja constante

Lema 1.3.9. (Lema de Hopf) Sejam $\Omega \subset \mathbb{R}^{n}$ um domínio e $\mathcal{L}$, como em (1.8), um operador uniformemente elíptico em $\Omega$ para o qual valem $\mathcal{L} u \geq 0$, onde $u \in C^{2}(\Omega)$, e $\frac{\left|b_{i}\right|}{\lambda}$ é limitado em $\Omega$, para cada $i$. ( $\lambda$ é o menor autovalor da matriz dos $\left(a_{i j}\right)$ ).

Seja $x_{0} \in \partial \Omega$ tal que:

(a) $u$ é contínua em $x_{0}$;

(b) $u\left(x_{0}\right)>u(x)$, para todo $x \in \Omega$;

(c) existe uma bola $\tilde{B} \subset \Omega$, com $x_{0} \in \partial \tilde{B}$.

Se a derivada normal exterior $\frac{\partial u}{\partial \nu}\left(x_{0}\right)$ de $u$ em $x_{0}$ existe então temos $\frac{\partial u}{\partial \nu}\left(x_{0}\right)>0$, onde $\nu$ é a normal unitária exterior a $\partial \Omega$ em $x_{0}$.

Prova: Pela hipótese (c), existe $\tilde{B}=B_{R}(y) \subset \Omega$, com $x_{0} \in \partial \tilde{B}$. Para $0<\rho<R$ e $\alpha>0$, definimos uma função $v(x)=e^{-\alpha r^{2}}-e^{-\alpha R^{2}}$, onde r é tomado como $r=\|x-y\|>\rho$ e $\alpha$ é uma constante a ser determinada.

Dadas as expressões de $\mathcal{L}$ e de $v(x)$, calculamos

$$
\mathcal{L} v(x)=e^{-\alpha r^{2}}\left[4 \alpha^{2} \sum_{\substack{i, j \\ i \neq j}} a_{i j}\left(x_{i}-y_{i}\right)\left(x_{j}-y_{j}\right)-2 \alpha \sum_{i}\left(a_{i i}+b_{i}\left(x_{i}-y_{i}\right)\right)\right]
$$


Como $\mathcal{L}$ é uniformemente elíptico, então $\sum a_{i j}\left(x_{i}-y_{i}\right)\left(x_{j}-y_{j}\right) \geq \lambda(x) r^{2}$. Além $i \neq j$

disto, segue da desigualdade de Cauchy-Schwarz que

$$
\sum_{i} b_{i}\left(x_{i}-y_{i}\right) \leq\|b\|\|x-y\|=\|b\| r
$$

Logo,

$$
\mathcal{L} v(x) \geq e^{-\alpha r^{2}}\left[4 \alpha^{2} \lambda(x) r^{2}-2 \alpha\left(\sum_{i} a_{i i}+\|b\| r\right)\right]
$$

Novamente pela elipticidade uniforme de $\mathcal{L}$, temos que $\lambda(x) \geq \lambda_{0}>0$ e os $a_{i i}$ são limitados. Por hipótese, os $b_{j}$ são também limitados. Portanto, existe uma constante $C_{1}$ real tal que:

$$
\mathcal{L} v(x) \geq e^{-\alpha r^{2}} \rho\left[\lambda_{0} \rho \alpha^{2}-C_{1} \alpha\right]
$$

Com isto, podemos encontrar um número positivo $\alpha$ de modo que $\mathcal{L} v \geq 0$ na região anelar $A=\{x ; \rho<\|x-y\|<R\}$.

Como $\partial B_{\rho}(y) \subset \Omega$, segue então da hipótese (b) que $u-u\left(x_{0}\right)<0$ em $\partial B_{\rho}(y)$. Além disto, $v(x)=e^{-\alpha\|x-y\|^{2}}-e^{-\alpha \cdot R^{2}}<0$ em A. Consequentemente, pela compacidade de $\partial B_{\rho}(y)$, existe $\epsilon>0$ tal que $w=u-u\left(x_{0}\right)+\epsilon v \leq 0$ em $\partial B_{\rho}(y)$. Procedendo de forma análoga, mostramos que $w=u-u\left(x_{0}\right)+\epsilon v \leq 0$ em $\partial B_{R}(y)$, pois $v=0$ em $\partial B_{R}(y)$. Juntando as informações obtidas, temos que $\mathcal{L}\left(u-u\left(x_{0}\right)+\epsilon v\right) \geq 0$ em $\mathrm{A}$, devido à expressão de $\mathcal{L}$, e $u-u\left(x_{0}\right)+\epsilon v \leq 0$ em $\partial A=\partial B_{\rho}(y) \bigcup \partial B_{R}(y)$. Pela Proposição 1.3.8, segue então que $w \leq 0$ em A, ou seja, $u(x)-u\left(x_{0}\right) \leq-\epsilon v(x), \forall x \in A$. Para $t<0$, tomando $x=x_{0}+t \nu$, temos:

$$
\frac{u\left(x_{0}+t \nu\right)-u\left(x_{0}\right)}{t} \geq-\epsilon \frac{v\left(x_{0}+t \nu\right)}{t} .
$$

Como $v\left(x_{0}\right)=e^{-\alpha\left\|x_{0}-y\right\|^{2}}-e^{-\alpha R^{2}}=0$, então o limite para $t$ tendendo a zero é:

$$
\frac{\partial u}{\partial \nu}\left(x_{0}\right) \geq-\epsilon \frac{\partial v}{\partial \nu}\left(x_{0}\right)
$$

Dada a expressão de $v(x)$, temos por definição:

$$
-\frac{\partial v}{\partial \nu}\left(x_{0}\right)=-\lim _{t \rightarrow 0} \frac{e^{-\alpha\left\|x_{0}+t \nu-y\right\|^{2}}-e^{-\alpha \cdot R^{2}}}{t}=2 \alpha e^{-\alpha \cdot R^{2}} R>0 .
$$


Portanto, $\frac{\partial u}{\partial \nu}\left(x_{0}\right)>0$

Teorema 1.3.10. (O Princípio do Máximo Forte) Sejam $\Omega \subset \mathbb{R}^{n}$ um domínio e $\mathfrak{L}$ como em (1.8), uniformemente elíptico em $\Omega$ para o qual valem $\mathfrak{L} u \geq 0$, onde $u \in C^{2}(\Omega)$, $e \frac{\left|b_{i}\right|}{\lambda}$ é limitado em $\Omega$, para cada $i$ ( $\lambda$ é o menor autovalor da matriz dos $\left(a_{i j}\right)$ ). Se u assume um máximo no interior da região $\Omega$, então u é constante em $\Omega$.

Prova: Suponhamos que u atinge seu máximo, $\mathrm{M}$, em um ponto $x_{0}$ do interior de $\Omega$. Seja $\mathrm{D}$ um domínio limitado cujo fecho está contido em $\Omega$ e tal que $x_{0} \in D$. Então, $M=\max _{\Omega} u=\max _{D} u$, e seja $D^{-}=\{x \in D ; u(x)<M\}$.

Suponhamos, por absurdo, que u é não-constante em D. Então, como $M=\max _{D} u, D^{-}$ pode ser visto como o complementar em D de $\{x \in D: u(x)=M\}=u^{-1}(\{M\})$, que é um conjunto fechado e, portanto, $D^{-}$é um aberto contido em D. Então, $\partial D^{-} \bigcap D \neq \emptyset, \partial D^{-} \bigcap D=\{x \in D ; u(x)=M\}$.

Escolhemos $x_{1} \in D^{-}$tal que $\operatorname{dist}\left(x_{1}, \partial D^{-}\right)<\operatorname{dist}\left(x_{1}, \partial D\right)$ e consideramos a maior bola B contida em $D^{-}$centrada em $x_{1}$. Então, $\partial B$ tem um ponto comum y com $\partial D^{-} \cap D$. Logo, $u(y)=M>u(x), \forall x \in B$, pois $y \in \partial D^{-} \cap D$ e $B \subset D^{-}$.

Pelo Lema 1.3.9, temos:

$\frac{\partial u}{\partial \nu}(y)>0$ e portanto, $\nabla u(y) \neq 0$, o que contraria o fato de y ser um ponto de máximo para $\mathrm{u}$ ( visto que $u(y)=M$ ). Portanto, $\mathrm{u}$ deve ser constante em D.

Observamos que podemos escolher $D=D_{R, \epsilon}$ como sendo o conjunto dos pontos que estão em uma bola de raio $\mathrm{R}$ centrada em $x_{0}$, cuja distância a $\partial \Omega$ é maior do $\epsilon>0$. Esta definição faz sentido para $\epsilon$ suficientemente pequenos, e pelo que acabamos de provar u é constante em $D=D_{R, \epsilon}$, para qualquer valor de $\mathrm{R}$ e para todos $\epsilon$ suficientemente pequenos. Com isto, temos então que u é constante em $\Omega$ (pois $x_{0} \in \Omega$ é arbitrário). 
1.4 Expressões do gradiente, da norma da $2^{a}$ forma fundamental em coordenadas e um resultado preliminar para a instabilidade do cilindro 17 1.4 Expressões do gradiente, da norma da $2^{\underline{a}}$ forma fundamental em coordenadas e um resultado preliminar para a instabilidade do cilindro

Os próximos resultados serão utilizados no capítulo 5, sendo que os dois primeiros, a pesar de serem clássicos na teoria de Geometria Diferencial, são deixados em geral como exercícios para o leitor.

Apresentamos então suas provas.

Lema 1.4.1. (Gradiente de uma função definida sobre uma superfície) Sejam $S$ uma superfície diferenciável regular e $f: S \longrightarrow \mathbb{R}$ uma função diferenciável. O gradiente de $f$ em $S$, denotado por $\nabla f$, é uma aplicação diferenciável $\nabla f: S \longrightarrow \mathbb{R}^{3}$ que associa a cada ponto $p \in S$ um vetor $\nabla f(p) \in T_{p}(S) \subset \mathbb{R}^{3}$ tal que:

$$
<\nabla f(p), v>_{p}=d f_{p}(v), \quad \forall v \in T_{p} S
$$

Se $E, F, G$ são os coeficientes da $1^{a}$ forma fundamental em uma parametrização $X: U \subset$ $\mathbb{R}^{2} \longrightarrow S$, então $\nabla f$ em $X(U)$ é dado por:

$$
\nabla f=\frac{f_{r} G-f_{s} F}{E G-F^{2}} X_{r}+\frac{f_{s} E-f_{r} F}{E G-F^{2}} X_{s}
$$

Prova:

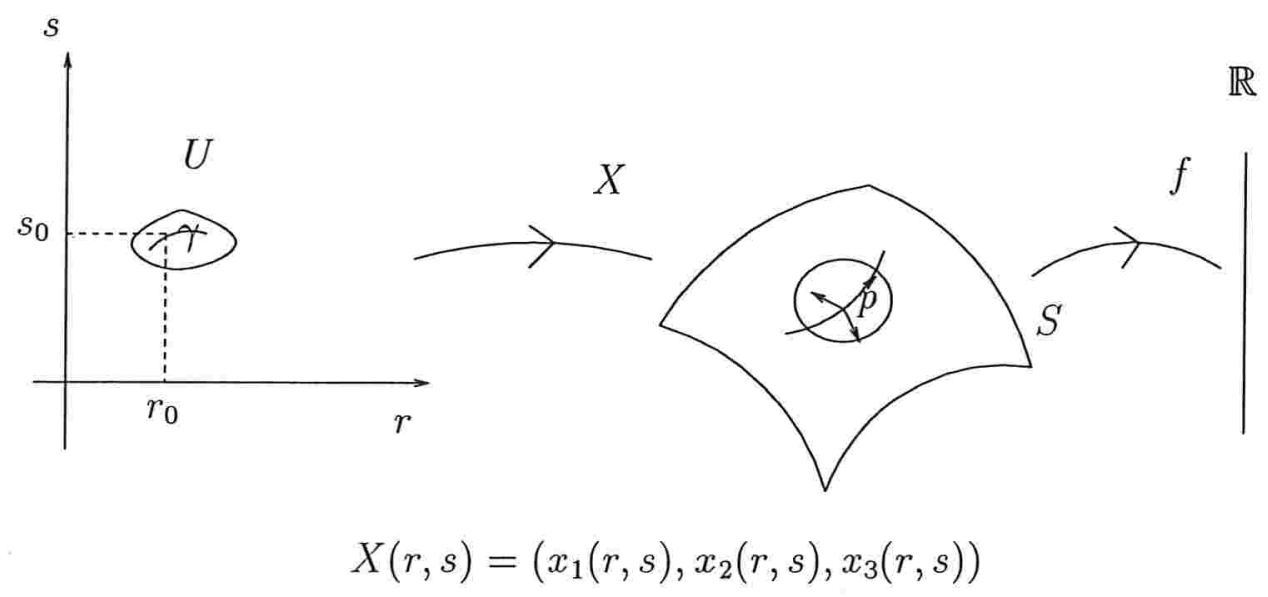

Figura 1.1: Representação do sistema de coordenadas para S 
Seja $p \in S$ arbitrário. Tomamos $X: U \longrightarrow S$ um sistema de coordenadas em torno de p. Logo, como $\nabla f(p) \in T_{p}(S)$, temos $\nabla f(p)=A X_{r}+B X_{s}$ onde A e B são as coordenadas de $\nabla f(p)$ no sistema. X.

Sejam $v \in T_{p} S$ arbitrário, $v=a X_{r}+b X_{s}$, e

$$
\gamma(t):=\left\{\begin{array}{l}
r=r_{0}+a t \\
s=s_{0}+b t
\end{array}\right.
$$

Logo, $v=\left.\frac{d}{d t}\right|_{t=0} X o \gamma(t)$ e, com isto,

$$
d f_{p} v=\left.\frac{d}{d t}\right|_{t=0} f(X o \gamma(t))=\left.\frac{d}{d t}\right|_{t=0}(f o X)\left(r_{0}+a . t, s_{0}+b . t\right)=a f_{r}+b f_{s},
$$

onde $f_{r}=\frac{\partial}{\partial r}(f o X), f_{s}=\frac{\partial}{\partial s}(f \circ X)$.

Por outro lado,

$$
\begin{aligned}
d f_{p}(v) & =<\nabla f(p), v>=<A X_{r}+B X_{s}, a X_{r}+b X_{s}> \\
& =A a E+(A b+B a) F+B b G .
\end{aligned}
$$

Ou seja, $a f_{r}+b f_{s}=A a E+(A b+B a) F+B b G, \forall a, b$.

Em particular, se

$$
\begin{aligned}
& a=1 \text { e } b=0 \quad \Longrightarrow \quad f_{r}=A E+B F \\
& a=0 \text { e } b=1 \quad \Longrightarrow \quad f_{s}=A F+B G .
\end{aligned}
$$

Do sistema

$$
\left\{\begin{array}{l}
f_{r}=A E+B F \\
f_{s}=A F+B G
\end{array}\right.
$$

segue que

$$
A=\frac{f_{r} G-f_{s} F}{E G-F^{2}}, \quad B=\frac{f_{s} E-f_{r} F}{E G-F^{2}}
$$

Portanto, $\nabla f=\frac{f_{r} G-f_{s} F}{E G-F^{2}} X_{r}+\frac{f_{s} E-f_{r} F}{E G-F^{2}} X_{s}, \quad \forall p \in S$.

Lema 1.4.2. (Expressão da norma da $2^{a}$ forma fundamental) Se $B$ é a $2^{a}$ forma fundamental de uma superfície $S$, então $\|B\|^{2}=k_{1}^{2}+k_{2}^{2}$, onde $k_{1}, k_{2}$ são as curvaturas principais de $S$. 


\subsection{Expressões do gradiente, da norma da $2^{\underline{a}}$ forma fundamental em} coordenadas e um resultado preliminar para a instabilidade do cilindro 19

Prova: Começamos mostrando que o traço tr de uma matriz é invariante por conjugação. De fato, sejam $E \in \mathbb{M}_{n}(\mathbb{K})$ uma matriz qualquer e $P^{-1} \cdot E . P$, com $\mathrm{P}$ ortogonal, uma matriz conjugada de E. Como $\operatorname{tr}(X Y)=\operatorname{tr}(Y X), \quad \forall X, Y \in \mathbb{M}_{n}(\mathbb{K})$ ( a prova deste resultado é trivial), temos então que:

$$
\operatorname{tr}\left(P^{-1} E P\right)=\operatorname{tr}\left(P^{-1}(E P)\right)=\operatorname{tr}\left(E P P^{-1}\right)=\operatorname{tr}(E)
$$

Agora, consideremos $\mathfrak{B}$ uma forma bilinear. Escrevemos $\mathfrak{B}$ em uma base ortonormal $\mathfrak{C}$ e seja $A=[\mathfrak{B}]_{\mathfrak{C}}$ a matriz que representa $\mathfrak{B}$ nesta base. Definimos o produto interno de duas matrizes $\in \mathbb{M}_{n}(\mathbb{K})$ por :

$<A_{1}, A_{2}>=\operatorname{tr}\left(A_{1} A_{2}^{t}\right)$ e portanto, $\left\|A_{1}\right\|^{2}=<A_{1}, A_{1}>=\operatorname{tr}\left(A_{1} A_{1}^{t}\right)$.

É fácil mostrar que este é, de fato, um produto interno. Além disto, a norma, dada pelo traço, não depende da escolha de base ortonormal. De fato, se tomarmos outra base ortonormal $\mathfrak{D}$, então $[\mathfrak{B}]_{\mathfrak{D}}$ é uma matriz conjugada de A (este é um resultado de álgebra linear ), isto é, $[\mathfrak{B}]_{\mathfrak{D}}=P^{-1} A P$, com $\mathrm{P}$ ortogonal, e como o traço é invariante por conjugação, então:

$$
\begin{aligned}
\left\|[\mathfrak{B}]_{\mathfrak{D}}\right\|^{2} & =<[\mathfrak{B}]_{\mathfrak{D}},[\mathfrak{B}]_{\mathfrak{D}}>=<P^{-1} A P, P^{-1} A P>=\operatorname{tr}\left(P^{-1} A P\left(P^{-1} A P\right)^{t}\right) \\
& =\operatorname{tr}\left(P^{-1} A P P^{-1} A^{t} P\right)=\operatorname{tr}\left(P^{-1} A A^{t} P\right)=\operatorname{tr}\left(A A^{t}\right)=<A, A> \\
& =<[\mathfrak{B}]_{\mathfrak{C}},[\mathfrak{B}]_{\mathfrak{C}}>=\left\|[\mathfrak{B}]_{\mathfrak{C}}\right\|^{2} .
\end{aligned}
$$

Usamos acima que como $\mathrm{P}$ é ortogonal, então $P^{-1}=P^{t}$.

Tomamos agora a forma bilinear $\mathfrak{B}$ como sendo a $2^{a}$ forma fundamental B de $\mathrm{S}$. Seja $\left\{e_{1}, e_{2}\right\}$ a base ortonormal formada pelas direções principais de S. Sabemos que, pela definição de B,

$$
\left\{\begin{array}{l}
B\left(e_{1}, e_{1}\right)=<S\left(e_{1}\right), e_{1}>=<k_{1} e_{1}, e_{1}>=k_{1} \\
B\left(e_{2}, e_{2}\right)=<S\left(e_{2}\right), e_{2}>=<k_{2} e_{2}, e_{2}>=k_{2} \\
B\left(e_{1}, e_{2}\right)=B\left(e_{2}, e_{1}\right)=<S\left(e_{1}\right), e_{2}>=k_{1}<e_{1}, e_{2}>=0
\end{array}\right.
$$

onde S é o operador de Weingarten (shape operator) de S.

Portanto,

$$
[B]_{\left\{e_{1}, e_{2}\right\}}=\left[\begin{array}{ll}
k_{1} & 0 \\
0 & k_{2}
\end{array}\right]
$$


Pelo que foi provado acima, segue então:

$$
\left\|[\mathfrak{B}]_{\left\{e_{1}, e_{2}\right\}}\right\|^{2}=\operatorname{tr}\left(\left[\begin{array}{ll}
k_{1} & 0 \\
0 & k_{2}
\end{array}\right]\left[\begin{array}{ll}
k_{1} & 0 \\
0 & k_{2}
\end{array}\right]\right)=\operatorname{tr}\left(\begin{array}{cc}
k_{1}^{2} & 0 \\
0 & k_{2}^{2}
\end{array}\right)=k_{1}^{2}+k_{2}^{2}
$$

Finalmente, terminamos esta seção apresentando o seguinte resultado de álgebra linear que será utilizado quando tratarmos da estabilidade do cilindro:

Lema 1.4.3. Sejam E espaço vetorial, $k \in \mathbb{R}$ fixo, $\alpha, \beta: E \longrightarrow \mathbb{R}$ aplicações tais que:

$$
\begin{aligned}
& \alpha(c v)=c^{k} \cdot \alpha(v) \\
& \beta(c v)=c^{k} \cdot \beta(v)
\end{aligned} \quad \forall c>0, \quad \text { e } \beta(v)>0 \quad \text { se } \quad v \neq 0 .
$$

Então, $\forall v \in E, v \neq 0$, temos que minimizar $\frac{\alpha(v)}{\beta(v)}$ equivale a minimizar $\alpha(v)$ sob $\beta(v)=1$.

Prova: Se provarmos que $\left\{\frac{\alpha(v)}{\beta(v)}: v \neq 0\right\}=\{\alpha(v): \beta(v)=1\}$, então seguirá que se existir o mínimo de um destes conjuntos então existe o mínimo do outro e são iguais. De fato, seja $x=\frac{\alpha(v)}{\beta(v)}$, para algum $v \in E, v \neq 0$. Tomando $c=\left(\frac{1}{\beta(v)}\right)^{1 / k}$, temos que $w=c v$ satisfaz $\beta(w)=\beta(c v)=c^{k} \beta(v)=\frac{1}{\beta(v)} \beta(v)=1 \mathrm{e}$ $\frac{\alpha(w)}{\beta(w)}=\frac{\alpha(c v)}{\beta(c v)}=\frac{c^{k} \alpha(v)}{c^{k} \beta(v)}=\frac{\alpha(v)}{\beta(v)}=x$. Ou seja, mostramos que $\exists w \in E \operatorname{com} \beta(w)=1 \mathrm{e}$ $\frac{\alpha(w)}{\beta(w)}=x$. Portanto, $x \in\{\alpha(v): \beta(v)=1\}$.

Seja agora $x=\alpha(v)$ para o qual $\beta(v)=1$ ( portanto, $v \neq 0$ ). Então,

$$
x=\alpha(v)=\frac{\alpha(v)}{\beta(v)} \text { e portanto, } x \in\left\{\frac{\alpha(v)}{\beta(v)}: v \neq 0\right\}
$$




\section{Caracterizações iniciais das soluções do problema variacional}

Consideremos novamente o problema que estamos abordando nesta dissertação:

"Sejam $P_{1}, P_{2} \subset \mathbb{R}^{3}$ dois planos paralelos. Procuramos determinar a forma da superfície S localizada entre eles, de classe $C^{2}$, orientável, mergulhada em $\mathbb{R}^{3}$, compacta e conexa com bordo $\partial S \subset P_{1} \bigcup P_{2}$ e que seja uma solução do problema variacional ( $\mathrm{P}$ ):

Minimizar o funcional área $A(S)$ sob a condição adicional que o volume $V(S)$, encerrado por $\mathrm{S}$, é constante $(V(S)=V)$."

Objetivo: Neste $2^{\underline{0}}$ capítulo mostramos, com base em [2], que uma tal S (solução de (P) ) tem curvatura média $\mathrm{H}$ constante e encontra os planos $P_{1}$ e $P_{2}$ perpendicularmente ao longo de seu bordo $\partial S$.

\subsection{Reformulação do problema variacional}

Como $\mathrm{S}$ foi suposta de classe $C^{2}$, então podemos considerar $\mathrm{S}$ dada localmente por $u=$ $u\left(x_{1}, x_{2}\right)$, onde $\left(x_{1}, x_{2}\right)$ pertence a um domínio $\Omega \subset \mathbb{R}^{2}$ e $u \in C^{2}(\Omega, \mathbb{R})$. Ou seja, S pode ser parametrizada localmente como gráfico de uma função $\mathrm{u}$, de classe $C^{2}$, por:

$$
X\left(x_{1}, x_{2}\right)=\left(x_{1}, x_{2}, u\left(x_{1}, x_{2}\right)\right)
$$

onde $\left(x_{1}, x_{2}\right) \in \Omega$. 
Com isto, definimos então $A(u)$ e $V(u)$ como, respectivamente a área e o volume de uma região em $\mathrm{S}$ dada por u. Sabemos da Geometria Diferencial que a área $A(u)$ e o volume $V(u)$ sob o gráfico de $u$ são dados por:

$$
A(u):=\int_{\Omega} \sqrt{1+\|\nabla u\|^{2}} d x
$$

onde $\nabla u=\left(u_{1}, u_{2}\right)$ e $u_{i}=\frac{\partial}{\partial x_{i}} u, i=1,2$,

$$
V(u):=\int_{\Omega} u d x
$$

Temos então o problema variacional $(\tilde{P})$ :

"Determinar $u \in C^{2}(\Omega, \mathbb{R})$ que minimiza $A(u)$ e satisfaz a condição adicional $V(u):=\int_{\Omega} u d x=c, c \in \mathbb{R}$ constante".

O problema $(\tilde{P})$ é a versão local do problema $(\mathrm{P})$ e, devido à natureza do resultado que queremos provar, sua análise será suficiente para cumprirmos o objetivo deste capítulo.

\subsection{As soluções são superfícies de curvatura média constante}

Mostraremos, com o próximo resultado, que se $u$ for uma solução de $(\tilde{P})$ então $\forall p \in S$ a vizinhança $u(\Omega)$ de $\mathrm{p}$ em S ( $\Omega$ e u tomados como na seção 2.1 ) tem curvatura média constante.

Teorema 2.2.1. Seja $u \in C^{2}(\Omega, \mathbb{R}), \Omega \subset \mathbb{R}^{2}$ aberto, uma solução do problema $(\tilde{P})$. Então, u satisfaz a equação diferencial:

$$
\frac{\left(1+u_{2}^{2}\right) u_{11}-2 u_{1} u_{2} u_{12}+\left(1+u_{1}^{2}\right) u_{22}}{\left(1+u_{1}^{2}+u_{2}^{2}\right)^{3 / 2}}=\lambda,
$$

onde $\lambda \in \mathbb{R}$ é constante e $u_{i}=\frac{\partial u}{\partial x_{i}}, u_{i j}=\frac{\partial^{2} u}{\partial x_{i} \partial x_{j}}, \quad i, j \in\{1,2\}$.

Consequentemente, $2 H(u)=\lambda$, onde $H(u)$ é a curvatura média da região $u(\Omega)$ em $S$ e portanto, $H(u)$ é constante. 
Prova: Consideramos variações de $u$ da forma $\left.u_{\epsilon}=u+\epsilon v, \epsilon \in\right]-\epsilon_{0}, \epsilon_{0}[$, com $v \in C_{c}^{2}(\Omega, \mathbb{R})$, ou seja, $v \in C^{2}(\Omega, \mathbb{R})$ e tem suporte compacto em $\Omega$.

Comentamos na introdução, que os pontos críticos do funcional área são também pontos críticos do funcional obtido com base no método dos multiplicadores de Lagrange. Definimos então, $\forall \epsilon \in]-\epsilon_{0}, \epsilon_{0}\left[\right.$, o funcional $J(\epsilon):=A\left(u_{\epsilon}\right)+\lambda V\left(u_{\epsilon}\right)$, onde $\lambda$ é o multiplicador de Lagrange, cuja existência é garantida pelo Lema 1.1.4.

Por hipótese temos que $u$ é uma solução do problema $(\tilde{P})$, ou seja, $A(u)=\min A\left(u_{\epsilon}\right)$ e $\left.V\left(u_{\epsilon}\right)=V(0), \forall \epsilon \in\right]-\epsilon_{0}, \epsilon_{0}\left[\right.$. Portanto, segue para a $1^{\underline{a}}$ variação de $\mathrm{J}$ que:

$$
\delta J:=\left.\frac{d}{d \epsilon} J(\epsilon)\right|_{\epsilon=0}=0 .
$$

Por outro lado, $\delta J:=\left.\frac{d}{d \epsilon}\left(\int_{\Omega}\left\{A\left(u_{\epsilon}\right)+\lambda V\left(u_{\epsilon}\right)\right\} d x\right)\right|_{\epsilon=0}$. Pela continuidade de $\frac{d}{d \epsilon}\left(A\left(u_{\epsilon}\right)+\lambda \cdot V\left(u_{\epsilon}\right)\right)$, visto que $A\left(u_{\epsilon}\right)$ e $V\left(u_{\epsilon}\right)$ são diferenciáveis com relação a $\epsilon$, segue que

$$
\delta J:=\left.\int_{\Omega} \frac{d}{d \epsilon}\left(\sqrt{1+\|\nabla u+\epsilon \nabla v\|^{2}}+\lambda(u+\epsilon v)\right)\right|_{\epsilon=0} d x, \text { devido às expressões (2.2) e }
$$

Portanto,

$$
\delta J=\int_{\Omega}\left(\frac{\nabla u \cdot \nabla v}{\sqrt{1+\|\nabla u\|^{2}}}+\lambda v\right) d x=0
$$

$\forall v \in C_{c}^{2}(\Omega, \mathbb{R})$

Por (1.5), temos que

$$
\int_{\Omega} \frac{\nabla u \cdot \nabla v}{\sqrt{1+\|\nabla u\|^{2}}} d x=\int_{\partial \Omega} v \frac{\nabla u \cdot \nu}{\sqrt{1+\|\nabla u\|^{2}}} d s-\int_{\Omega} v \operatorname{div}\left(\frac{\nabla u}{\sqrt{1+\|\nabla u\|^{2}}}\right) d x
$$

onde $\nu$ e ds foram definidos no Teorema1.2.1.

Como $v \in C_{c}^{2}(\Omega, \mathbb{R})$, então $v \equiv 0$ em $\partial \Omega$. Portanto, (2.6) se torna

$$
\int_{\Omega} \frac{\nabla u \cdot \nabla v}{\sqrt{1+\|\nabla u\|^{2}}} d x=-\int_{\Omega} v \operatorname{div}\left(\frac{\nabla u}{\sqrt{1+\|\nabla u\|^{2}}}\right) d x
$$

Substituindo (2.7) em (2.5) temos:

$\delta J=\int_{\Omega}\left\{-\operatorname{div}\left(\frac{\nabla u}{\sqrt{1+\|\nabla u\|^{2}}}\right)+\lambda\right\} v d x=0, \forall v \in C_{c}^{2}(\Omega, \mathbb{R})$. Pelos Lemas $1.1 .2 \mathrm{e}$ 
Observação 1.1.3, segue que:

$$
\lambda=\operatorname{div}\left(\frac{\nabla u}{\sqrt{1+\|\nabla u\|^{2}}}\right) .
$$

Por definição,

$$
\begin{aligned}
\operatorname{div}\left(\frac{\nabla u}{\sqrt{1+\|\nabla u\|^{2}}}\right) & =\frac{\partial}{\partial x_{1}}\left(\frac{u_{1}}{\sqrt{1+u_{1}^{2}+u_{2}^{2}}}\right)+\frac{\partial}{\partial x_{2}}\left(\frac{u_{2}}{\sqrt{1+u_{1}^{2}+u_{2}^{2}}}\right) \\
& =\frac{\left(1+u_{2}^{2}\right) u_{11}-2 u_{1} u_{2} u_{12}+\left(1+u_{1}^{2}\right) u_{22}}{\left(1+u_{1}^{2}+u_{2}^{2}\right)^{3 / 2}}
\end{aligned}
$$

Com isto, (2.4) está provada.

Seja $p \in S$ arbitrário. Usando (2.1) como sendo a parametrização da região $u(\Omega)$, vizinhança de $\mathrm{p}$ em $\mathrm{S}$, segue após alguns cálculos:

região $u(\Omega)$.

$2 H(u)=\frac{\left(1+u_{2}^{2}\right) u_{11}-2 u_{1} u_{2} u_{12}+\left(1+u_{1}^{2}\right) u_{22}}{\left(1+u_{1}^{2}+u_{2}^{2}\right)^{3 / 2}}$, onde $H(u)$ é a curvatura média da

Portanto, $2 H(U)=\lambda$, onde $\lambda \in \mathbb{R}$ é uma constante, ou seja, a região $u(\Omega)$ em $S$ tem curvatura média constante.

Observação 2.2.2. Sejam $p, q \in S$ dois pontos arbitrários. Sejam $U$ e $V$ duas vizinhanças de $p$ e q, respectivamente, tais que $U \cap V \neq \emptyset$. Sempre é possivel escolher $U$ e $V$ deste modo pois, como $S$ é compacta e conexa, temos $d(p, q)<\infty$ e portanto, basta tomar $U=B_{\frac{2}{3} d(p, q)}(p) \bigcap S, \quad V=B_{\frac{2}{3} d(p, q)}(q) \bigcap S$.

Além disto, existem $\Omega_{1}, \Omega_{2} \subset \mathbb{R}^{2}$ de modo que $U=u\left(\Omega_{1}\right)$ e $V=u\left(\Omega_{2}\right)$. Pelo Teorema 2.2.1 temos que $u\left(\Omega_{1}\right)$ e $u\left(\Omega_{2}\right)$ têm curvatura média constante, por exemplo, $H\left(u\left(\Omega_{1}\right)\right)=c_{1}$ e $H\left(u\left(\Omega_{2}\right)\right)=c_{2}$. Como $U \cap V \neq \emptyset$, ou seja, $u\left(\Omega_{1}\right) \bigcap u\left(\Omega_{2}\right) \neq \emptyset$, segue que $c_{1}=c_{2}$ em $\left\{u\left(\Omega_{1}\right) \bigcap u\left(\Omega_{2}\right)\right\}$ e portanto, $c_{1}=c_{2}$.

Como p e q são arbitrários e, deste modo, U e V também os são, concluímos que $\mathrm{S}$ tem curvatura média constante. 
2.3 As soluções são superfícies que encontram os planos $P_{1}$ e $P_{2}$ perpendicularmente.

2.3 As soluções são superfícies que encontram os planos $P_{1}$ e $P_{2}$ perpendicularmente.

Nesta seção mostraremos que se u for solução do problema $(\tilde{P})$ então $\forall p \in \partial S$ a vizinhança $u(\Omega)$ de $\mathrm{p}$ em $\mathrm{S}$ encontra o plano $P_{i}$, que contém o ponto $\mathrm{p}$, perpendicularmente ao longo de seu bordo.

Teorema 2.3.1. Seja $\Omega:=\left\{\left(x_{1}, x_{2}\right) \in \mathbb{R}^{2}: x_{1}^{2}+x_{2}^{2}<r, x_{2} \geq 0\right\}, \quad r$ arbitrário $e$ suficientemente pequeno de modo que $u\left(\Omega \bigcap\left\{x_{2}=0\right\}\right) \subset \partial S$. Sejam $u \in C^{2}(\Omega, \mathbb{R})$ uma solução de $(\tilde{P}), \partial_{1} \Omega:=\Omega \bigcap\left\{x_{2}=0\right\}, \partial_{2} \Omega:=\partial \Omega-\partial_{1} \Omega$. Então, vale para a derivada normal exterior no bordo $\partial_{1} \Omega$ :

$$
\frac{\partial u}{\partial \nu}=\nabla u \cdot \nu=0
$$

onde $\nu$ denota a normal unitária exterior em $\partial \Omega$.

Prova: Consideremos as variações de $u$ da forma $\left.\left.u_{\epsilon}=u+\epsilon . v, \epsilon \in\right]-\epsilon_{0}, \epsilon_{0}\right]$, com $v \in C^{2}(\Omega, \mathbb{R}),\left.v\right|_{\partial_{2} \Omega}=0$. Procedendo analogamente a.o Teorema 2.2.1, definimos

$$
J(\epsilon)=A(\epsilon)+\lambda V(\epsilon)
$$

Por hipótese, temos $A(u)=\min A(\epsilon)$ e $V(u)=V(0), \forall \epsilon \in]-\epsilon_{0}, \epsilon_{0}[$. Logo,

$$
\delta J=\left.\frac{d}{d \epsilon} J(\epsilon)\right|_{\epsilon=0}=0
$$

Por outro lado, segue dos cálculos feitos no Teorema 2.2.1 que:

$$
\delta J=\int_{\Omega}\left\{-\operatorname{div}\left(\frac{\nabla u}{\sqrt{1+\|\nabla u\|^{2}}}\right)+\lambda\right\} v d x+\int_{\partial \Omega} v\left(\frac{\nabla u \cdot \nu}{\sqrt{1+\|\nabla u\|^{2}}}\right) d s,
$$

$\forall v \in C^{2}(\Omega, \mathbb{R}),\left.\quad v\right|_{\partial_{2} \Omega}=0$, onde $\nu$ e ds foram definidos no Teorema 1.2.1.

No Teorema 2.2 .1 mostramos que $\lambda=\operatorname{div}\left(\frac{\nabla u}{\sqrt{1+\|\nabla u\|^{2}}}\right)$ e portanto, a $1^{\underline{a}}$ integral de (2.10) é nula. Consequentemente, juntando (2.9) e (2.10), temos:

$$
\delta J=\int_{\partial \Omega} v \frac{\nabla u \cdot \nu}{\sqrt{1+\|\nabla u\|^{2}}} d s=0
$$

$\forall v \in C^{2}(\Omega, \mathbb{R}),\left.\quad v\right|_{\partial_{2} \Omega}=0$ 
Como $\partial \Omega=\partial_{1} \Omega \bigcup \partial_{2} \Omega,\left\{\partial_{1} \Omega \cap \partial_{2} \Omega\right\}$ tem medida nula, $\left.v\right|_{\partial_{2} \Omega}=0$, temos
$0=\delta J=\int_{\partial_{1} \Omega} v \frac{\nabla u \cdot \nu}{\sqrt{1+\|\nabla u\|^{2}}} d s+\int_{\partial_{2} \Omega} v \frac{\nabla u \cdot \nu}{\sqrt{1+\|\nabla u\|^{2}}} d s=\int_{\partial_{1} \Omega} v \frac{\nabla u \cdot \nu}{\sqrt{1+\|\nabla u\|^{2}}} d s$,

$\forall v \in C^{2}(\Omega, \mathbb{R})$

Pelos Lema 1.1.2 e Observação 1.1.3, segue de (2.12) que:

$\frac{\nabla u \cdot \nu}{\sqrt{1+\|\nabla u\|^{2}}}=0$ em $\partial_{1} \Omega$ e portanto, $\nabla u \cdot \nu=0$ em $\partial_{1} \Omega$.

A diferenciabilidade de u segue da hipótese de $u \in C^{2}(\Omega, \mathbb{R})$ e, desta forma, sabemos do Cálculo Diferencial que $\frac{\partial u}{\partial \nu}=\nabla u \cdot \nu$.

Portanto, $\frac{\partial u}{\partial \nu}=\nabla u \cdot \nu=0$ em $\partial_{1} \Omega$.

Antes de concluirmos o resultado de que a superfície $\mathrm{S}$ encontra os planos $P_{1}$ e $P_{2}$ ao longo de seu bordo $\partial S$, formalizamos este conceito:

Definição 2.3.2. Dizemos que uma superfície $S$ encontra um plano $\pi$ perpendicularmente ao longo de seu bordo $\partial S$ se o campo normal unitário ao longo do bordo da componente conexa que intercepta $\pi$ pertencer ao plano $\pi$.

Sejam $p \in \partial S$ arbitrário, $\Omega, \partial_{1} \Omega, \partial \Omega$ como no Teorema 2.3.1 e $u(\Omega)$ a vizinhança de $\mathrm{p}$ em $\mathrm{S}$ tal que $u\left(\Omega \bigcap\left\{x_{2}=0\right\}\right) \subset \partial S$. Associamos ao ponto p o sistema de coordenadas ortogonais de $\mathbb{R}^{3}$ e o representamos por $\left(O, x_{1}, x_{2}, x_{3}\right)$, onde $x_{3}=u\left(x_{1}, x_{2}\right)$, e de modo que $p=u(0,0)$, com $(0,0)$ a origem do plano $O x_{1} x_{2}$. Tomamos (2.1) como sendo a parametrização de $u(\Omega)$ e o plano $P_{i}, i=1$ ou 2 , que contém o ponto $\mathrm{p}$, como sendo o plano $O x_{1} x_{3}$, ou seja, $x_{2}=0$.

Seja $\nu$ a normal unitária exterior a $\partial_{1} \Omega$. Pelas considerações acima, $\nu=(0,1)$ e temos a seguinte ilustração ( ver Figura 2.1 ).

Pelo Teorema 2.3.1, temos que $\nabla u \cdot \nu=0$ em $\partial_{1} \Omega$. Mas, $\nabla u \cdot \nu=u_{1} .0+u_{2} \cdot 1$, onde $u_{i}=\frac{\partial}{\partial x_{i}} u, i=1,2$. Portanto segue que

$$
u_{2}(p)=0
$$

$\forall p \in \partial S$, pois p foi tomado arbitrariamente. 


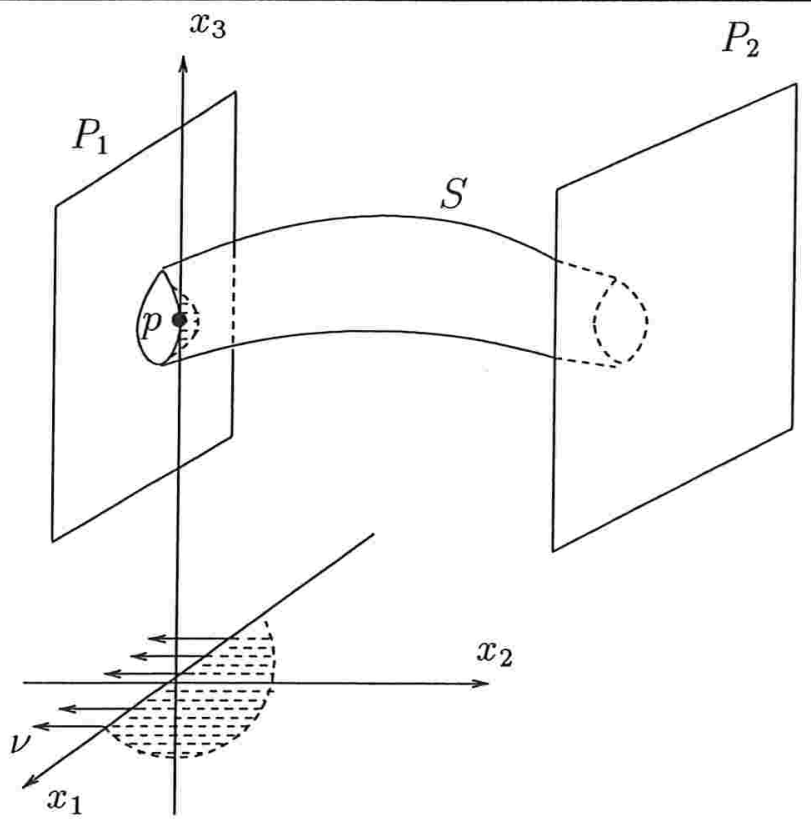

Figura 2.1: Representação do sistema de coordenadas para $p \in \partial S$

Mas, (2.13) implica, pela Definição 2.3.2, que S encontra o plano $P_{i}$ perpendicularmente ao longo de seu bordo, pois calculando a expressão do campo normal unitário $N(p)$, tomando a parametrização (2.1) de $S$, temos:

$N(p)=\left(-\frac{u_{1}}{\sqrt{1+u_{1}^{2}+u_{2}^{2}}},-\frac{u_{2}}{\sqrt{1+u_{1}^{2}+u_{2}^{2}}}, \frac{1}{\sqrt{1+u_{1}^{2}+u_{2}^{2}}}\right)_{p}=\left(-\frac{u_{1}}{\sqrt{1+u_{1}^{2}}}, 0, \frac{1}{\sqrt{1+u_{1}^{2}}}\right)_{p} \in P_{i}$, $\forall p \in \partial S$

Conclusão: Mostramos até agora que as soluções do problema variacional $(\mathrm{P})$ são superfícies de curvatura média constante e que encontram os planos $P_{1}$ e $P_{2}$ perpendicularmente ao longo de seu bordo. 



\section{Superfícies rotacionais como soluções do problema variacional}

Neste capítulo, provaremos o princípio de reflexão de Alexandrov adaptado ao caso em que a superfície $\mathrm{S}$ é uma superfície mergulhada em $\mathbb{R}^{3}$ de classe $C^{2}$, orientável, compacta, que está localizada entre os planos paralelos $P_{1}, P_{2} \subset \mathbb{R}^{3}$, com bordo $\partial S \subset P_{1} \bigcup P_{2}$ e, além disto, é suposta conexa. Segundo este resultado, se $\mathrm{S}$ tiver ainda curvatura média constante, então seguirá que S é uma superfície rotacionalmente simétrica ao redor de um eixo perpendicular a $P_{1}$ e $P_{2}$. Para isto, tomamos como base a referência [1], na qual $\mathrm{S}$ é uma superfície com condições mais gerais do que aquelas supostas acima. Observamos também que este resultado valerá, em particular, para as soluções do problema (P), visto que elas satisfazem todas as hipóteses necessárias.

Trataremos ainda neste capítulo da equação da curvatura média constante, e neste momento utilizaremos [5] e [13] como referências básicas.

\subsection{Condições para uma superfície ser rotacionalmen- te simétrica}

Começamos apresentando o seguinte lema, que será utilizado na conclusão do resultado principal deste capítulo.

Lema 3.1.1. Seja $S$ uma superfície mergulhada em $\mathbb{R}^{3}$, de classe $C^{2}$, orientável, conexa e 
compacta, que está localizada entre os planos paralelos $P_{1}, P_{2}$ e com bordo $\partial S \subset P_{1} \bigcup P_{2}$. Se $S$ tem em cada direção um plano de simetria, que corta os planos $P_{1}, P_{2}$ perpendicularmente, então existe um eixo de simetria de $S$ e ele é perpendicular a $P_{1}$ e a $P_{2}$.

Prova: Consideramos um plano $\mathrm{P}$ qualquer paralelo a $P_{1}$ e a $P_{2}$ e localizado entre eles. O primeiro passo da prova consiste em mostrar que $S \cap P$ são círculos concêntricos.

Geometricamente, sabemos que as intersecções dos planos de simetria de S com P dão as retas de simetria de $S \cap P$. Segue então, da hipótese de $\mathrm{S}$ ter em cada direção um plano de simetria, que $S \cap P$ tem, em cada direção, uma reta de simetria. Além disto, todas as retas de simetria de $S \cap P$ se cruzam no centro de gravidade $\sigma$ de $S \cap P$. De fato, se refletirmos $S \cap P$ em relação a uma reta de simetria $r_{1}$ qualquer, então surge uma figura equivalente, que deve ter portanto o mesmo centro de gravidade $\sigma$. Desta forma, temos que $\sigma \in r_{1}$. Tomando outra reta de simetria $r_{2}$ qualquer, concluímos de forma análoga que $\sigma \in r_{2}$ e portanto, $\sigma \in r_{1} \bigcap r_{2}$. Como $r_{1}$ e $r_{2}$ são arbitrárias, segue que todas as retas de simetria de $S \cap P$ se cruzam em $\sigma$.

Seja $p \in S \cap P$ qualquer. Refletindo p por uma reta de simetria $r_{i}$ qualquer de $S \cap P$, então sua imagem $p_{i}$ também pertence a $S \bigcap P$.

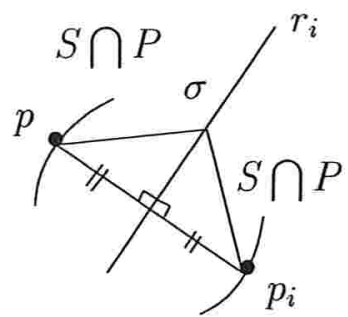

Figura 3.1: Imagem da reflexão do ponto $p \in S \cap P$ em relação à reta $r_{i}$

Como $\sigma \in r_{i}$ e $\operatorname{dist}\left(p, r_{i}\right)=\operatorname{dist}\left(p_{i}, r_{i}\right)$, então $\operatorname{dist}(p, \sigma)=\operatorname{dist}\left(p_{i}, \sigma\right)$. Como $r_{i}$ é arbitrária, então $\operatorname{dist}(p, \sigma)=\operatorname{dist}\left(p_{i}, \sigma\right), \forall i$, ou seja, as imagens $p_{i}$ pertencem a uma circunferência de centro $\sigma$ e raio $\operatorname{dist}(p, \sigma)$. No entanto, se tomarmos $\tilde{p} \in S \cap P, \tilde{p} \neq p$, obteremos analogamente que as imagens $\tilde{p}_{i}$ de $\tilde{p}$ por $r_{i}$ pertencem a uma circunferência de centro $\sigma$ e raio $\operatorname{dist}(\tilde{p}, \sigma)$. Como p e $\tilde{p}$ são arbitrários, concluímos que $S \bigcap P$ consiste eventualmente de círculos concêntricos, e isto prova o primeiro passo.

O segundo passo é mostrar que todos os $\sigma$, nas distintas intersecções $S \bigcap P$, pertencem 
a uma mesma reta. De fato, os centros de gravidade $\sigma$, nas distintas intersecções, pertencem a todos os planos de simetria, pois pertencem a todas as retas de simetria que, por sua vez, são obtidas pela intersecção dos planos de simetria com P. Além disto, sabemos que dois planos de simetria de $\mathrm{S}$ se interceptam em uma reta. Consequentemente, todos os $\sigma$ pertencem a uma mesma reta $\mathrm{r}$. Por hipótese, temos que os planos de simetria são perpendiculares a $P_{1}$ e a $P_{2}$ e, com isto, $\mathrm{r}$ também o é. Tomamos então $\mathrm{r}$ como sendo o eixo de simetria de S. Após o Princípio de Reflexão de Alexandrov, que será tratado a seguir, veremos ainda que $S \cap P$ é formada por um único círculo (ver Observação 3.2.3).

Passemos a tratar do resultado principal desta seção:

\subsection{O Princípio de Reflexão de Alexandrov}

Teorema 3.2.1. Seja $S$ uma superfície mergulhada em $\mathbb{R}^{3}$, de classe $C^{2}$, orientável, conexa e compacta, que está localizada entre os planos paralelos $P_{1}, P_{2}$ e com bordo $\partial S \subset P_{1} \cup P_{2}$. Se tivermos ainda que a curvatura média $H$ de $S$ é constante, então $S$ é rotacionalmente simétrica ao redor de um eixo perpendicular a $P_{1}$ e a $P_{2}$.

Prova: A prova deste resultado é um pouco extensa e para que a suas etapas fiquem bem claras vamos dividi-la em três partes, sendo que na primeira descrevemos o método do Princípio para a superfície que estamos considerando; na segunda, tratamos da equação da curvatura média constante e, finalmente, na terceira, juntamos as duas primeiras para concluirmos a tese deste teorema.

1ํㅡ parte: O Método do Princípio de Reflexão

Tomamos uma reta $\mathrm{R}$ qualquer em um dos planos $P_{1}$ ou $P_{2}$, e $\mathrm{P}$ um plano perpendicular à reta $\mathrm{R}$ obtido da seguinte maneira: consideramos um feixe $\mathrm{F}$ de planos perpendiculares a R e deslocamos $\mathrm{F}$ em direção à superfície $\mathrm{S}$; escolhemos para ser o plano $\mathrm{P}$ aquele plano de $\mathrm{F}$ que primeiro tangenciar a superfície $\mathrm{S}$. Desta forma, temos que $\mathrm{P}$ é perpendicular a $P_{1}$ e a $P_{2}$ e é um plano suporte de $\mathrm{S}$ (ver Figura 3.2 ).

Conforme observamos na introdução da dissertação, S delimita um sólido compacto K. Movemos então o plano P paralelamente de modo a cruzar o sólido K. Em cada posição de 


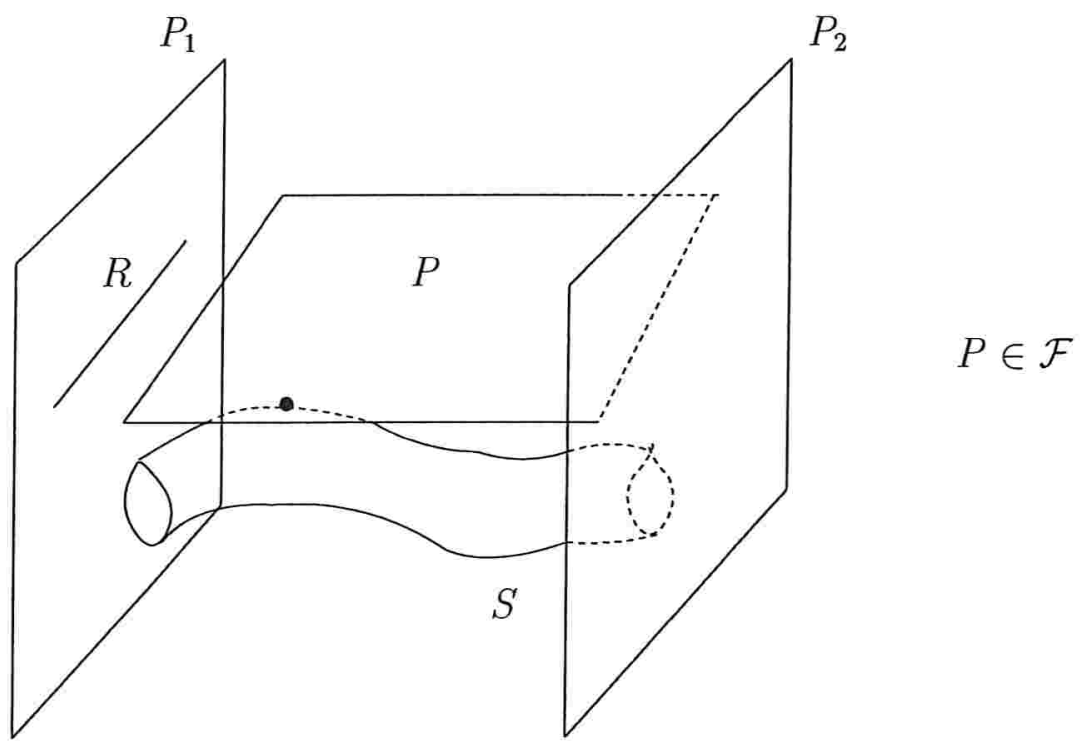

Figura 3.2: Posição da reta $\mathrm{R}$ e do plano $\mathrm{P}$ em relação aos planos $P_{1}$ e $P_{2}$ e à superfície $\mathrm{S}$

$\mathrm{P}$, refletimos em relação a $\mathrm{P}$ a parte de $\mathrm{S}$ já cruzada e a denotamos por $S_{r}$. Para pequenos deslocamentos de $\mathrm{P}$, temos que $S_{r}$ pertence ao interior de $\mathrm{K}$.

Matematicamente, podemos descrever o processo como:

Seja $P^{\delta}$ o plano obtido quando deslocamos $\mathrm{P}$ a uma distância $\delta$, de modo a cruzar K. Sejam $\left\{P^{\delta}\right\}$ a família de planos paralelos a $\mathrm{P}$ assim obtidos, e $\left\{S_{r}^{\delta}\right\}$ a família formada pelas partes refletidas de $\mathrm{S}$ em relação a $P^{\delta}$. Definimos:

$$
\delta^{*}:=\sup \left\{\delta: \delta=\operatorname{dist}\left(P^{\delta}, P\right), \quad S_{r}^{\delta} \subset K\right\}
$$

Continuamos com o processo de reflexão até o momento em que obtemos $P^{\delta^{*}}$ e $S_{r}^{\delta^{*}}$ que, por motivo de simplificação, chamamos novamente de $\mathrm{P}$ e $S_{r}$.

Pela hipótese de compacidade de $\mathrm{S}$, temos que para este $\delta^{*}, S_{r}$ e a parte $\tilde{S}$ de $\mathrm{S}$ que está do mesmo lado de $\mathrm{P}$ que $S_{r}$ têm, no mínimo, um ponto $x_{0}$ em comum. Além disto, vemos geometricamente que $S_{r}$ e $\tilde{S}$ têm o mesmo plano tangente neste ponto $x_{0}$. Por esta razão, chamamos $x_{0} \circ 1^{\circ}$ ponto de contato (ver Figura 3.3).

No nosso caso, em que $\mathrm{S}$ está entre os planos $P_{1}, P_{2}$, podem ocorrer as seguintes possibilidades quanto à posição de $x_{0}$ em relação a $\mathrm{S}$, que representamos em um corte de $\mathrm{S}$ com um plano perpendicular a $P_{1}$, a $P_{2}$ e a $\mathrm{P}$, e que contém o ponto $x_{0}$. 


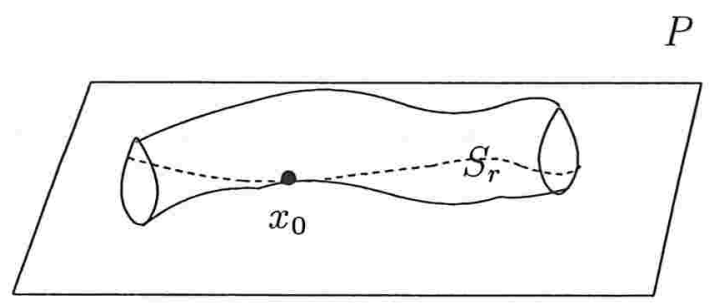

Figura 3.3: Determinação do $1^{\circ}$ ponto de contato $x_{0}$ pela reflexão de um pedaço de $\mathrm{S}$ em relação ao plano P

(i) $x_{0}$ é um ponto do interior de $\mathrm{S}$, que denotamos por $\stackrel{o}{S}$ :

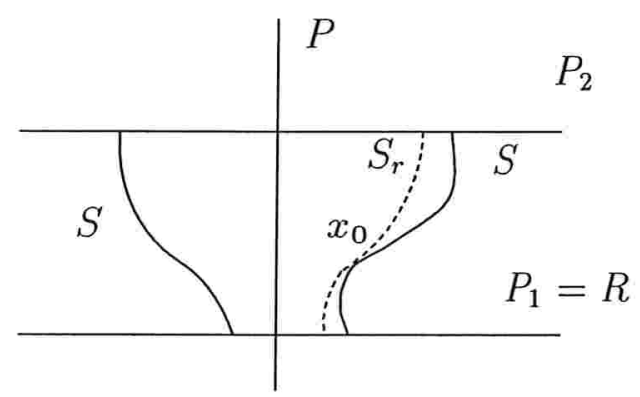

Figura 3.4: Representação da possibilidade em que $x_{0} \in \stackrel{o}{S}$

(ii) $x_{0} \in \partial S$ e portanto, $x_{0}$ pertence a um dos planos $P_{i}$ :

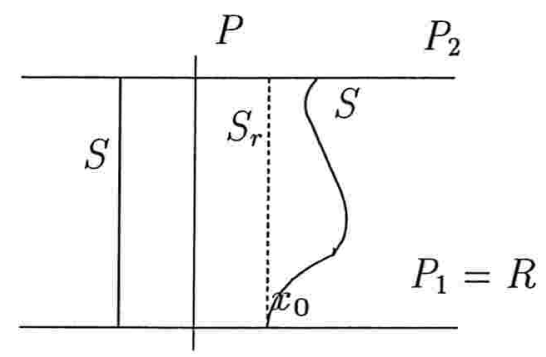

Figura 3.5: Representação da possibilidade em que $x_{0} \in \partial S$

$\mathrm{Na}$ referência [1], que tomamos como base para o estudo do Princípio de Reflexão, vemos que o caso (ii) acima não ocorre. Para continuarmos então como na prova de Alexandrov, quando $x_{0} \in \partial S$ refletimos $S$ em relação ao plano $P_{i}$ que contém $x_{0}$ e, desta forma, $x_{0}$ passa a ser um ponto do interior da superfície $S^{\prime}$ composta por $\mathrm{S}$ e pela imagem de $\mathrm{S}$ refletida por $P_{i}$ (ver Figura 3.6). 


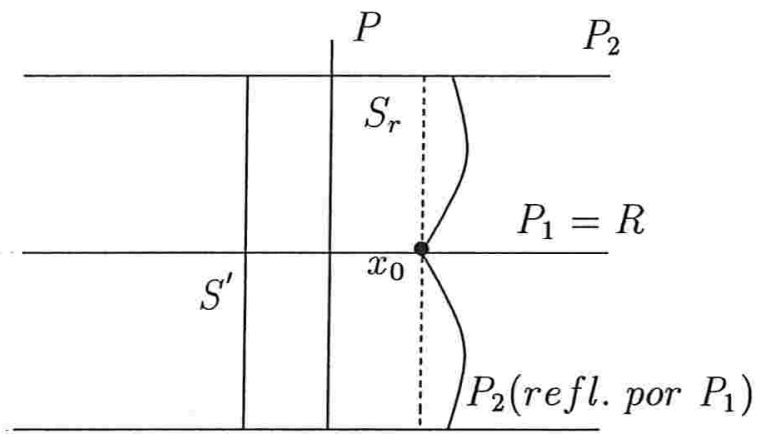

Figura 3.6: Reflexão de $\mathrm{S}$ e $S_{r}$ em relação a $P_{1}$

Por construção, a superfície $S^{\prime}$ também satisfaz todas as hipóteses do Teorema 3.2.1 e, com isto, o caso (ii) pode ser tratado como sendo o caso (i). Além disto, se mostrarmos que $S^{\prime}$ é rotacional, então $S$ também o será. Com relação à posição de $x_{0}$ em relação a $S_{r}$, há, então, duas possibilidades para estudarmos:

(a) $x_{0}$ pertence ao interior de $S_{r}$, que denotamos por $\stackrel{o}{S}_{r}$. Este caso pode ser ilustrado com os casos (i) e (ii) acima.

(b) $x_{0} \in \partial S_{r}$, formado pelas curvas de intersecção de $S_{r}$ com P.

Ilustramos a seguir o caso (b).

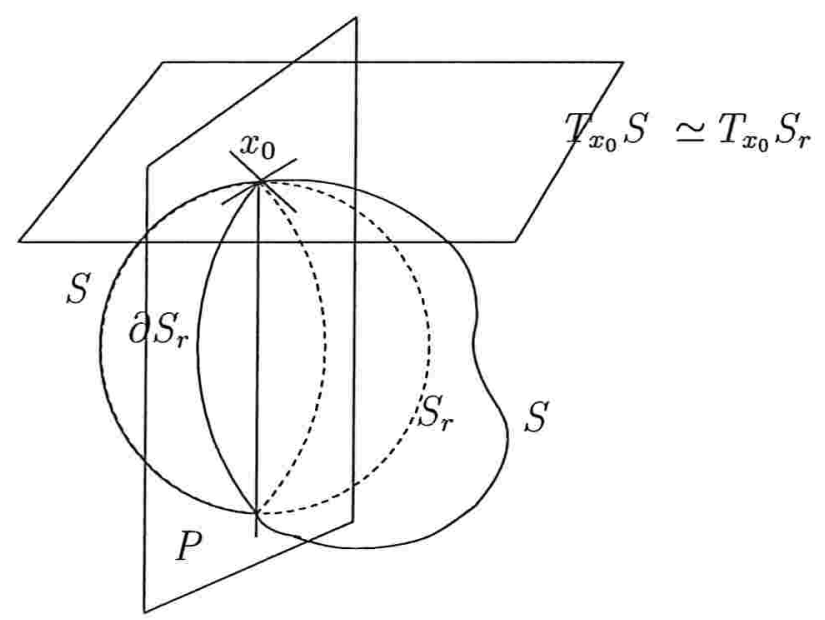

Figura 3.7: Representação da possibilidade em que $x_{0} \in \partial S_{r}$

$\mathrm{Ou}$, representando em um corte de $\mathrm{S}$ com um plano perpendicular a $P_{1}$, a $P_{2}$, a $\mathrm{P}$ e que contém o ponto $x_{0}$ (ver Figura 3.8). 


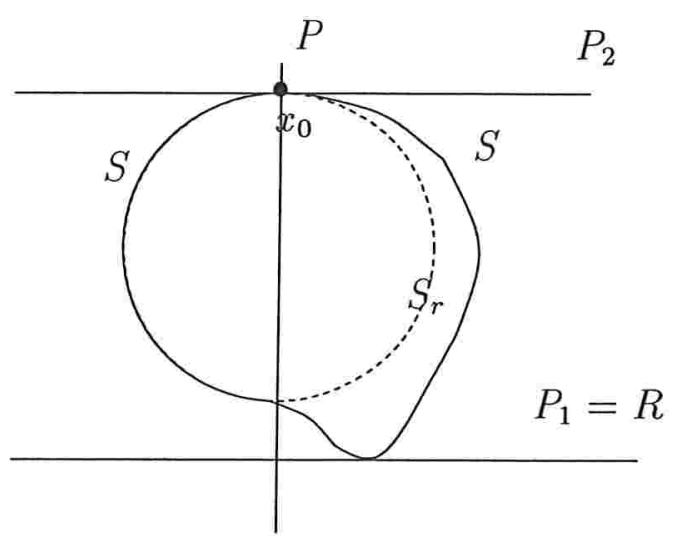

Figura 3.8: Outra representação para a possibilidade em que $x_{0} \in \partial S_{r}$

Com base nas hipóteses de $S$, temos que para ambos os casos (a) e (b), podemos parametrizar uma vizinhança de $x_{0}$ em S e $S_{r}$ como gráficos de funções $u=u\left(x_{1}, x_{2}\right), \quad \bar{u}=$ $\bar{u}\left(x_{1}, x_{2}\right)$, respectivamente, $u, \bar{u} \in C^{2}(B, \mathbb{R})$, onde B é tomado convenientemente nos casos (a) e (b), e B é uma vizinhança de $x_{0}$ em $T_{x_{0}} S \simeq T_{x_{0}} S_{r}$.

Consideramos a função diferença $v: B \rightarrow \mathbb{R}, v:=u-\bar{u}$. Então, como $x_{0}$ é um ponto comum de $\mathrm{S}$ e $S_{r}$, por construção, temos que $v\left(x_{0}\right)=0$.

Para o caso (a), tomamos $B:=\left\{x=\left(x_{1}, x_{2}\right) \in \mathbb{R}^{2} /\left\|x-x_{0}\right\|<\rho\right\}$, vizinhança de $x_{0}$ em $T_{x_{0}} S, \rho \in \mathbb{R}_{+}^{*}$ arbitrário e suficientemente pequeno. Além disto, podemos tomar, neste caso,

$$
v(x) \leq 0, \quad \forall x \in B,
$$

de acordo com a escolha de orientação em $\mathbb{R}^{3}$. Isto é possível pois, como $x_{0}$ é o primeiro ponto comum entre $S_{r}$ e $\tilde{S}$, então a vizinhança de $x_{0}$ em $S_{r}$ pertence ainda ao interior de $\mathrm{K}$.

Para o caso (b), tomamos $B:=\left\{x=\left(x_{1}, x_{2}\right) \in \mathbb{R}^{2} /\|x\|<\rho, x_{2} \geq 0\right\}, \quad \rho \in \mathbb{R}_{+}^{*}$ arbitrário e suficientemente pequeno. Já sabemos que para ambos os casos, $\mathrm{S}$ e $S_{r}$ têm o mesmo plano tangente $T_{x_{0}} S$ em $x_{0}$; mas, especificamente para (b), $T_{x_{0}} S$ é perpendicular a P. Podemos então associar a $x_{0}$ o seguinte sistema de coordenadas: escolhemos $x_{0}$ para ser a origem e $x_{1}, x_{2}$ como sendo as coordenadas em $T_{x_{0}} S$,por exemplo, $x_{1}$ na direção da reta obtida pela intersecção de $T_{x_{0}} S$ com $\mathrm{P}$ e $x_{2}$ na direção da reta perpendicular a $x_{1}$; escolhemos $x_{3}$ na direção e sentido da normal a $S$ em $x_{0}$ que aponta para o interior do sólido K. Com esta escolha, as vizinhanças de $x_{0}$ em S e $S_{r}$ são dadas pelos correspondentes 
$x_{3}=u\left(x_{1}, x_{2}\right), x_{3}=\bar{u}\left(x_{1}, x_{2}\right)$, respectivamente, onde $\left(x_{1}, x_{2}\right) \in B$, e B é uma vizinhança de $x_{0}$ em $T_{x_{0}} S$. Além disto, segue também desta escolha que:

$$
v(x) \leq 0, \quad \forall x \in B
$$

pois a vizinhança de $x_{0}$ em $S_{r}$ pertence ao interior de K.

Como S e $S_{r}$ têm o mesmo plano tangente em $x_{0}$, então

$$
\frac{\partial v}{\partial x_{i}}\left(x_{0}\right)=0, \quad \text { para } i=1,2 .
$$

$2^{\underline{a}}$ parte: A equação da curvatura média constante

Por hipótese (do Teorema 3.2.1), temos que a superfície $\mathrm{S}$ tem curvatura média constante. Como o processo de reflexão de uma superfície mantém sua orientação e sua curvatura média, temos que, por construção, $\mathrm{S}$ e $S_{r}$ têm a mesma curvatura média constante, digamos:

$$
H(u)=H(\bar{u})=H_{0}
$$

onde $H(u)$ ( conforme Teorema 2.2.1 ) está definida por:

$$
2 H(u)=\frac{\left(1+u_{2}^{2}\right) u_{11}-2 u_{1} u_{2} u_{12}+\left(1+u_{1}^{2}\right) u_{22}}{\left(1+u_{1}^{2}+u_{2}^{2}\right)^{3 / 2}}
$$

$u_{i}=\frac{\partial u}{\partial x_{i}}, \quad u_{i j}=\frac{\partial^{2} u}{\partial x_{i} \partial x_{j}}, \quad i, j \in\{1,2\}$.

Observamos que a expressão (3.5) depende apenas das derivadas primeira e segunda de u, ou seja, $2 H(u)=F(x, q, r)$, onde $q=\left(q_{i}\right)_{i}, q_{i}=\frac{\partial u}{\partial x_{i}}, r=\left(r_{i j}\right)_{i, j}, r_{i j}=\frac{\partial^{2} u}{\partial x_{i} \partial x_{j}}$, onde $\mathrm{F}$ é uma função diferenciável definida em $B \times \mathbb{R}^{2} \times \mathbb{R}^{4}$ dada explicitamente por:

$$
F(x, q, r)=\frac{\left(1+q_{2}^{2}\right) r_{11}-2 q_{1} q_{2} r_{12}+\left(1+q_{1}^{2}\right) r_{22}}{\left(1+\|q\|^{2}\right)^{3 / 2}} .
$$

Como B é um conjunto convexo, e portanto, segue que $u(B)$ também o é, podemos construir entre $\mathrm{S}$ e $S_{r}$ uma familia convexa de superfícies $\left\{u^{t}\right\}$, definida por:

$$
u^{t}(x):=t u(x)+(1-t) \bar{u}(x)=t v(x)+\bar{u}(x),
$$

$\forall x \in B, \forall t \in[0,1]$, onde $\mathrm{u}, \bar{u}$ foram definidas na seção anterior. 
Definimos agora

$$
2 H(t):=2 H\left(u^{t}\right)=F(x, t q+(1-t) \bar{q}, t r+(1-t) \bar{r}),
$$

onde $\bar{q}=\left(\bar{q}_{i}\right)_{i}, \overline{q_{i}}=\frac{\partial \bar{u}}{\partial x_{i}}, \bar{r}=\left(\bar{r}_{i j}\right)_{i, j}, \quad \bar{r}_{i j}=\frac{\partial^{2} \bar{u}}{\partial x_{i} \partial x_{j}}$. Com isto, temos que $H(t) \in$ $C^{1}([0,1], \mathbb{R})$.

Pelo Teorema Fundamental do Cálculo e pelas expressões (3.8),(3.7) e (3.4) segue que:

$$
\int_{0}^{1} \dot{H}(t) d t=H(1)-H(0)=H(u)-H(\bar{u})=0 .
$$

Por outro lado, usando a definição de $H(t)$ em (3.8), e aplicando a Regra da Cadeia , temos por (3.9) que

$$
\mathfrak{L} v:=\sum_{i, j=1}^{2}\left(\int_{0}^{1} \frac{\partial F}{\partial \tilde{r}_{i j}}(\xi) d t\right) v_{i j}+\sum_{i=1}^{2}\left(\int_{0}^{1} \frac{\partial F}{\partial \tilde{q}_{i}}(\xi) d t\right) v_{i}=0
$$

onde $v=u-\bar{u}, \quad v_{i}=\frac{\partial v}{\partial x_{i}}, \quad v_{i j}=\frac{\partial^{2} v}{\partial x_{i} \partial x_{j}}, \xi=\xi(t)=$

$=(x, t q+(1-t) \bar{q}, t r+(1-t) \bar{r}), \quad \tilde{q}_{i}=\frac{\partial u^{t}}{\partial x_{i}}, \quad \tilde{r}_{i j}=\frac{\partial^{2} u^{t}}{\partial x_{i} \partial x_{j}}$.

$\mathfrak{L}$ é um operador que pode ser escrito como

$$
\mathfrak{L} v:=\sum_{i, j=1}^{2} a_{i j}(x) v_{i j}(x)+\sum_{i=1}^{2} b_{i}(x) v_{i}(x)
$$

com $a_{i j}(x)=a_{j i}(x), \forall x \in B$, onde os coeficientes são dados por:

$$
a_{i j}(x)=\int_{0}^{1} \frac{\partial F}{\partial \tilde{r}_{i j}}(\xi) d t \quad \text { e } \quad b_{i}(x)=\int_{0}^{1} \frac{\partial F}{\partial \tilde{q}_{i}}(\xi) d t .
$$

Utilizando as equações $u_{i}^{t}=t u_{i}+(1-t) \bar{u}_{i}, \quad u_{i j}^{t}=t u_{i j}+(1-t) \bar{u}_{i j}$, calculamos as expressões dos coeficientes. Por exemplo,

$$
\begin{aligned}
& a_{11}(x)=\int_{0}^{1} \frac{1+\left(u_{2}^{t}\right)^{2}}{\left(1+\left(u_{1}^{t}\right)^{2}+\left(u_{2}^{t}\right)^{2}\right)^{3 / 2}} d t, \quad a_{22}(x)=\int_{0}^{1} \frac{1+\left(u_{1}^{t}\right)^{2}}{\left(1+\left(u_{1}^{t}\right)^{2}+\left(u_{2}^{t}\right)^{2}\right)^{3 / 2}} d t, \\
& a_{12}(x)=-\int_{0}^{1} \frac{u_{1}^{t} u_{2}^{t}}{\left(1+\left(u_{1}^{t}\right)^{2}+\left(u_{2}^{t}\right)^{2}\right)^{3 / 2}} d t=a_{21}(x), \text { e assim por diante. }
\end{aligned}
$$

Mostramos agora que a EDP (3.10) é elíptica (ver Definição 1.3.3). De fato, como o integrando que aparece na expressão de $a_{11}(x)$ é positivo, então $a_{11}(x)>0$. Além disto, 
definindo o produto interno $<,>$ de duas funções $a(t)$ e $b(t)$ quaisquer por $<a(t), b(t)>=\int_{0}^{1} \frac{a(t) b(t)}{\left(1+\left(u_{1}^{t}(t)\right)^{2}+\left(u_{2}^{t}(t)\right)^{2}\right)^{3 / 2}} d t \quad$ (onde $u_{1}^{t}$ e $u_{2}^{t}$ estão definidas acima) e usando a desigualdade de Cauchy-Schwarz, resulta que:

$$
\begin{aligned}
\left(a_{12}(x)\right)^{2} & =\left(\int_{0}^{1} \frac{u_{1}^{t} u_{2}^{t}}{\left(1+\left(u_{1}^{t}\right)^{2}+\left(u_{2}^{t}\right)^{2}\right)^{3 / 2}} d t\right)^{2}=<u_{1}^{t}, u_{2}^{t}>^{2} \leq \\
& \leq\left(\int_{0}^{1} \frac{\left(u_{1}^{t}\right)^{2}}{\left(1+\left(u_{1}^{t}\right)^{2}+\left(u_{2}^{t}\right)^{2}\right)^{3 / 2}} d t\right)\left(\int_{0}^{1} \frac{\left(u_{2}^{t}\right)^{2}}{\left(1+\left(u_{1}^{t}\right)^{2}+\left(u_{2}^{t}\right)^{2}\right)^{3 / 2}} d t\right)< \\
& <\left(\int_{0}^{1} \frac{1+\left(u_{1}^{t}\right)^{2}}{\left(1+\left(u_{1}^{t}\right)^{2}+\left(u_{2}^{t}\right)^{2}\right)^{3 / 2}} d t\right)\left(\int_{0}^{1} \frac{1+\left(u_{2}^{t}\right)^{2}}{\left(1+\left(u_{1}^{t}\right)^{2}+\left(u_{2}^{t}\right)^{2}\right)^{3 / 2}} d t\right) \\
& =a_{11}(x) \cdot a_{22}(x) .
\end{aligned}
$$

Como o resultado anterior vale $\forall x \in B$, então segue pela Definição 1.3.3 que o operador $\mathfrak{L}$, definido em (3.10), é elíptico em B. Além disto, como o que foi provado acima independe dos $u^{t}$ utilizados e, consequentemente, independe também da solução $v$, isto prova nossa afirmação de que a $\operatorname{EDP}(3.10)$ é elíptica.

Mostraremos agora que o operador $\mathfrak{L}$ é uniformemente elíptico. Já sabemos que o operador simétrico $\mathfrak{L}$ é elíptico. Além disto, com os mesmos argumentos que usamos para provar que $a_{11}(x)>0$, concluímos que $a_{22}(x)>0$. Desta forma, temos que o menor autovalor $\lambda(x)$ da matriz dos $a_{i j}(x), \lambda(x)$ calculado em (1.9), é tal que $\lambda(x)>0$. Logo, pela Observação 1.3.4 temos que vale (1.11). Em seguida, observamos que os integrandos dos coeficientes $a_{i j}(x), b_{i}(x), i, j \in\{1,2\}$, envolvem apenas as derivadas parciais de $u^{t}$ até $2^{\underline{a}}$ ordem. Como $u^{t}(x):=t u(x)+(1-t) \bar{u}(x), \quad t \in[0,1]$, então as derivadas parciais de $u^{t}$ até $2^{\underline{a}}$ ordem são contínuas com respeito a $t$ (devido à sua expressão) e também são contínuas com respeito a $x$ ( pois $\mathrm{u}, \bar{u} \in C^{2}(B, \mathbb{R})$ ), de onde segue que os integrandos de $a_{i j}(x), b_{i}(x), i, j \in\{1,2\}$, são contínuos com respeito às variáveis $t$ e $x$. Tomando o compacto $K=[0,1]$ ( portanto, $m(\partial K=0)$ ), X como sendo o espaço topológico B e f como sendo os integrandos das funções $a_{i j}(x), b_{i}(x)$, segue pelo Lema 1.3.1 que as funções $a_{i j}(x), b_{i}(x), \forall i, j$, são contínuas com respeito a $x, \forall x \in B$. Pelas expressões (1.9) e (1.10), o menor e o maior autovalor de $\left(a_{i j}(x)\right), \lambda(x)$ e $\Lambda(x)$, respectivamente, dependem somente dos $a_{i j}(x)$ e, consequentemente, são funções contínuas em $x, \forall x \in B$. Como $\lambda(x)>0, \forall x \in B$, então $\frac{\Lambda(x)}{\lambda(x)}$ é uma função contínua em $x, \forall x \in B$. Lembramos que o conjunto B foi definido na parte anterior em cada caso (a) ou (b). Para o caso (a) consideramos o compacto 
$B^{*}:=\left\{x=\left(x_{1}, x_{2}\right) \in \mathbb{R}^{2} /\left\|x-x_{0}\right\| \leq \tilde{\rho}\right\}, \quad \tilde{\rho} \in \mathbb{R}_{+}^{*}, \tilde{\rho}<\rho$ e portanto, $B^{*} \subset B$. Para o caso (b) consideramos o compacto $B^{*}:=\left\{x=\left(x_{1}, x_{2}\right) \in \mathbb{R}^{2} /\|x\| \leq \tilde{\rho}, \quad x_{2} \geq\right.$ $0\}, \quad \tilde{\rho} \in \mathbb{R}_{+}^{*}, \tilde{\rho}<\rho$ e também aqui vale $B^{*} \subset B$. Como $\frac{\Lambda(x)}{\lambda(x)}$ é contínua no conjunto $\mathrm{B}$ de ambos os casos, pela Observação 1.3.6 segue que $\frac{\Lambda(x)}{\lambda(x)}$ é limitado em $B^{*}$ e portanto, $\mathfrak{L}$ é uniformemente elíptico em $B^{*}$ (para ambos os casos). Como na definição de $\mathrm{B} \rho$ era arbitrário, então tomando $\rho:=\tilde{\rho}$ e redefinindo para ambos para os casos (a) e (b) os conjuntos $B=\operatorname{int} B^{*}\left(B^{*}\right.$ do caso (a) ) e $B:=\left\{x=\left(x_{1}, x_{2}\right) \in \mathbb{R}^{2} /\|x\|<\tilde{\rho}, \quad x_{2} \geq 0\right\}$, respectivamente, segue que o operador $\mathfrak{L}$, dado em (3.11), é uniformemente elíptico em $B$.

Procedendo de forma análoga ao que foi feito acima, mostramos também que os $\frac{\left|b_{i}(x)\right|}{\lambda(x)}, \forall i \in\{1,2\}$, são limitados nos conjuntos B redefinidos acima para os casos (a) e (b).

3a parte: Aplicação dos Princípios do Máximo para a geometria da superfície $S$

Verificamos agora que, com os resultados obtidos nas duas partes anteriores, estamos em condições de aplicar os chamados Princípios do Máximo para obtermos mais caracterizações da superfície $\mathrm{S}$.

Observamos que para ambos os casos (a) e (b) da $1^{\underline{a}}$ parte, mostramos que o operador $\mathfrak{L}$, dado em (3.11) é uniformemente elíptico em B e vale $\frac{\left|b_{i}(x)\right|}{\lambda(x)}$ é limitado em $\mathrm{B}, \forall i$, conforme vimos no final da parte anterior. Para a função $v$ introduzida na $1^{\stackrel{a}{ }}$ parte ainda vale que $\mathfrak{L} v=0$, por (3.10), e portanto, $\mathfrak{L} v \geq 0$ para ambos os casos (a) e (b).

Especificamente para o caso (a), ainda temos, por (3.1), que $v(x) \leq 0, \forall x \in B$, e $v\left(x_{0}\right)=0$, onde $x_{0}$ pertence ao interior de B. Ou seja, a função $v$ assume seu máximo no interior da região B. Pelo Teorema 1.3.10, segue que $v$ é constante. Mas, $v\left(x_{0}\right)=0$ e portanto, $v=u-\bar{u}=0$ em B, isto é, $u \equiv \bar{u}$ em B.

Especificamente para o caso (b), ainda temos por (3.2) que $v(x) \leq 0, \forall x \in B$, e $v\left(x_{0}\right)=0$, onde $x_{0}$ pertence à fronteira de $\mathrm{B}$. Ou seja, $v$ assume seu máximo na fronteira de $\mathrm{B}$. Também temos que $\mathrm{v}$ é contínua em $x_{0}$, pois $v=u-\bar{u}$ e $u, \bar{u} \in C^{2}(B, \mathbb{R})$. Pela forma como tomamos o conjunto $\mathrm{B}$ do caso (b), é óbvio que existe uma bola $\tilde{B} \subset B$ 
com $x_{0} \in \partial \tilde{B}$. Pelo Lema 1.3.9 segue que se a derivada $\frac{\partial v}{\partial \nu}\left(x_{0}\right)$ existir, onde $\nu$ é a normal unitária exterior, então $\frac{\partial v}{\partial \nu}\left(x_{0}\right)=\nabla v \cdot \nu>0$, o que é absurdo, pois por (3.3) devemos ter $\frac{\partial v}{\partial \nu}\left(x_{0}\right)=0$. A contradição surgiu do fato de termos suposto que $v$ assume seu máximo na fronteira de $\mathrm{B}$, pois esta é a única condição que depende basicamente da construção feita nos passos anteriores, e as outras condições são mais gerais. Desta forma, $v$ assume seu máximo no interior de $\mathrm{B}$ e novamente pelo Teorema 1.3.10, concluímos de forma análoga que $u \equiv \bar{u}$ em B.

Com isto, mostramos que $S_{r}$ e $\tilde{S}$ coincidem em uma vizinhança de $x_{0}$ para ambos os casos (a) e (b).

Como não exigimos inicialmente que o sólido $\mathrm{K}$ fosse convexo, então $S_{r}$ pode naturalmente consistir de várias componentes conexas.

Vamos mostrar agora que cada componente conexa $S_{r}^{i}$ de $S_{r}$ que contém o ponto de contato $x_{0}$ está inteiramente contida em $S$.

Para isto, consideramos o conjunto:

$$
Q=\left\{x \in \mathbb{R}^{3}: x \text { é ponto comum de } S \text { e } S_{r}^{i}\right\}=S \bigcap S_{r}^{i} .
$$

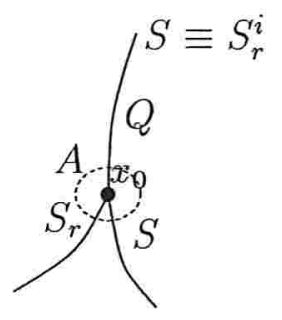

Figura 3.9: Representação do conjunto Q

Logo, Q é aberto e fechado de $S_{r}^{i}$. De fato,

- Q é aberto de $S_{r}^{i}$ :

Seja $p \in Q$. Queremos mostrar que existe vizinhança $V$ de $p$ tal que $V \subset Q$, onde $V=A \bigcap S_{r}^{i}$, A aberto de $\mathbb{R}^{3}$.

Se $p \in \stackrel{\circ}{Q}$ ( interior de Q ), então basta tomar $A=\stackrel{o}{Q}$ e portanto, $p \in V=A \bigcap S_{r}^{i}=$ $\stackrel{\circ}{Q} \cap S_{r}^{i} \subset Q$, pois $\stackrel{\circ}{Q} \subset Q \subset S_{r}^{i}$. 
Se $p \in \partial Q$, então existe vizinhança $U$ de $\mathrm{p}$ em $\mathbb{R}^{3}$ tal que $U \cap Q \neq \emptyset$ e $U \cap Q^{c} \neq \emptyset$, onde $Q^{c}$ denota o complementar de $\mathrm{Q}$ em $\mathbb{R}^{3}$ e na qual p pode ser visto como sendo o primeiro ponto de contato $x_{0}$ de $S$ e $S_{r}^{i}$. Logo, pelo que mostramos há pouco para $x_{0}$, concluímos que existem uma vizinhança $\mathrm{A}$ de $p=x_{0}$ em $\mathbb{R}^{3}$ e uma vizinhança $\mathrm{V}$ de $x_{0}, V=A \bigcap S_{r}^{i}$, na qual S e $S_{r}^{i}$ coincidem. Por (3.12), temos que $V \subset Q$.

- $\underline{\text { é fechado de } S_{r}^{i}}$ :

Da análise de $p \in \partial Q$ também podemos concluir que $\partial Q=\emptyset$, pois mostramos que se $p \in \partial Q$ então existe vizinhança $V$ de p, $V \subset Q$, ou seja, $p \in \stackrel{o}{Q}$. Logo, o fecho de $Q$ é tal que: $\bar{Q}=Q \bigcup \partial Q=Q$ e portanto, $Q$ é fechado de $\mathbb{R}^{3}$. Além disto, como $Q=S \cap S_{r}^{i}$, então $Q \subset S_{r}^{i}$ e, consequentemente, $Q \cap S_{r}^{i}=Q$. Portanto, Q é fechado de $S_{r}^{i}$.

Também temos que $Q \neq \emptyset$, pois $x_{0} \in Q$ e já garantimos a existência de, no mínimo, um pontó $x_{0}$. Portanto, $Q \equiv S_{r}^{i}$, onde $S_{r}^{i}$ é uma componente conexa de $S_{r}$ que contém $x_{0}$. Mas, como $Q=S \cap S_{r}^{i}$, segue que cada componente conexa $S_{r}^{i}$ que contém $x_{0}$ está inteiramente contida em $\mathrm{S}$.

A seguir apresentamos algumas ilustrações com as quais concluíremos que $\mathrm{P}$ é um plano de simetria para S. Consideramos a situação mais simples possível, onde $x_{0} \in \stackrel{\circ}{S_{r}}$ (ver Figura 3.10) :

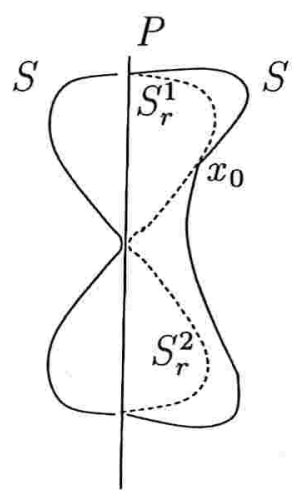

Figura 3.10: Situação da figura 3.10 após termos mostrado que $S_{r}^{1} \subset S$

Com o que mostramos acima, $S_{r}^{1}$ está inteiramente contida em S, conforme a Figura 3.11 a seguir.

Portanto, deve existir um ponto de contato $x_{0}$, que pertence simultaneamente ao bordo 


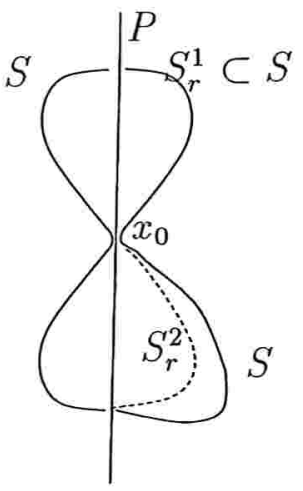

Figura 3.11: Situação da figura 3.10 após termos mostrado que $S_{r}^{1} \subset S$ e $S_{r}^{2} \subset S$

de $S_{r}^{1}$ e ao bordo de $S_{r}^{2}$. Mas também vimos acima que, quando $x_{0}$ é um ponto de bordo, vale $S_{r}^{2} \subset S$, o que nos leva à conclusão de que se existe $x_{0} \in \stackrel{\circ}{S}_{r}$ então a situação inicial se reduz à Figura 3.12.

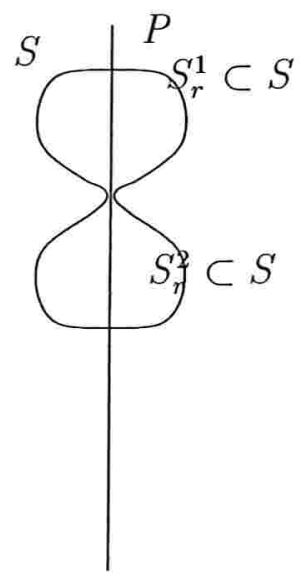

Figura 3.12: Representação de uma situação em que $x_{0}$ é um ponto de contato de $S_{r}^{1}$ e S

Ou seja, a parte $\tilde{S}$ de $\mathrm{S}$ que está do mesmo lado de $\mathrm{P}$ que $S_{r}^{i}$ coincide com a imagem de S pela reflexão com relação ao plano $\mathrm{P}$.

Portanto, provamos que $\mathrm{S}$ possui um plano de simetria $\mathrm{P}$ que corta $P_{1}, P_{2}$ perpendicularmente.

Como $R$ e $P$ eram arbitrários, então tomando este processo em todas as direções concluímos que $S$ tem em cada direção um plano de simetria perpendicular a $P_{1}$ e a $P_{2}$. Pelo Lema 3.1.1, segue ainda que o eixo de simetria de $\mathrm{S}$ é perpendicular a $P_{1}$ e a $P_{2}$, o 
que conclui a prova do Teorema 3.2.1.

Observação 3.2.2. Na conclusão acima usamos algumas ilustrações para mostrar que o Princípio de Reflexão determinava que o plano $P$ era um plano de simetria de $S$. Para isto, utilizamos a hipótese de $S$ ser uma superfície conexa.Se $S$ fosse desconexa então poderíamos imaginar a situação ilustrada na Figura 3.13.

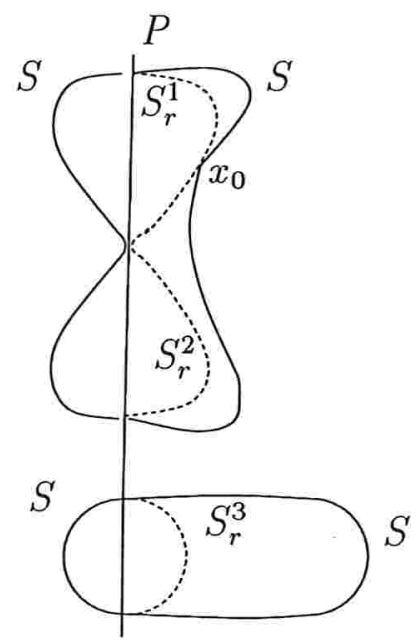

Figura 3.13: Representação de uma situação em que S é desconexa

Com isto, não conseguiríamos proceder como nas ilustrações anteriores para mostrar que $\mathrm{P}$ é um plano de simetria de $\mathrm{S}$. Na verdade, só garantiríamos que cada componente conexa tem um plano de simetria.

Observação 3.2.3. Na prova do Lema 3.1.1, mostramos que $S \cap P$ consistia eventualmente de vários círculos concêntricos. Usaremos a seguinte ilustração para justificar que o Princípio de Reflexão determina que $S \cap P$ é formado por um único círculo. Consideramos a seguinte representação em que $S \cap P$ consiste de dois círculos concêntricos. 


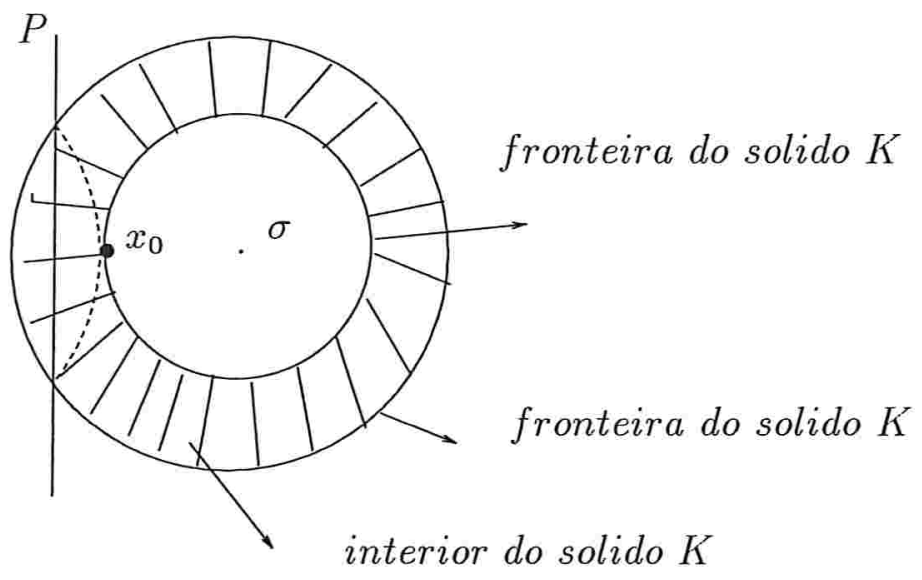

Figura 3.14: Representação de uma situação em que $S \bigcap P$ consiste de dois círculos concêntricos

Seja P um plano tomado como no método descrito na $1^{\underline{a}}$ parte. Pelo Princípio de Reflexão de Alexandrov, segue que P é então um plano de simetria de S, o que é absurdo, como podemos ver na ilustração anterior.

Conclusão: Mostramos até agora que as soluções do problema variacional (P) são superfícies $\mathrm{S}$ com curvatura média $\mathrm{H}$ constante e encontram os planos $P_{1}, P_{2}$ perpendicularmente ao longo de seu bordo $\partial S$. Utilizando a hipótese de que elas são conexas mostramos ainda que elas são rotacionalmente simétricas ao redor de um eixo perpendicular a $P_{1}$ e a $P_{2}$. 
Capítulo 4

\section{Superfícies rotacionais em $\mathbb{R}^{3}$ com curvatura média constante}

Neste capítulo será possível, com base no trabalho de Delaunay [11], classificarmos as soluções conexas do problema variacional (P) pois, como já sabemos, elas são superfícies rotacionais com curvatura média constante e portanto, são exatamente as chamadas " $\mathrm{Su}$ perfícies de Delaunay". Elas são geradas por curvas descritas pelos focos de seções cônicas que rolam, sem deslizar, ao longo de um eixo e compreendem o cilindro, o catenóide, o nodóide, o ondulóide, bem como a esfera e o plano.

Observamos que o resultado obtido por Delaunay em [11] não exige a hipótese de que a superfície esteja localizada entre dois planos paralelos e tenha seu bordo contido nos planos; além disto, ele vale para superfícies com condições mais gerais do que as exigidas em nosso trabalho. Em razão disto, utilizamos toda a classificação feita por Delaunay e concluímos que, no nosso caso, as soluções de $(\mathrm{P})$ são determinadas por aqueles pedaços das superfícies de Delaunay limitados entre os planos paralelos $P_{1}$ e $P_{2}$ e com bordo contido neles. 


\subsection{Algumas propriedades das superfícies rotacionais} em $\mathbb{R}^{3}$

Obtemos nesta seção algumas propriedades gerais das superfícies rotacionais que valem, portanto, para as soluções conexas do problema variacional $(\mathrm{P})$.

Consideramos então $J \subset \mathbb{R}$ um intervalo aberto e $\gamma(t)=(x(t), 0, z(t)), \gamma \in C^{2}\left(J, \mathbb{R}^{3}\right)$, uma curva regular em $\mathbb{R}^{3}$, ou seja,

$$
\dot{\gamma}(t)=\frac{d}{d t} \gamma(t) \neq 0, \quad \forall t \in J
$$

com

$$
x(t)>0, \quad \forall t \in J .
$$

Seja $\mathrm{S}$ a superfície obtida quando giramos a curva $\gamma$ em relação ao eixo $z$. Tomamos para S a parametrização usual das superfícies rotacionais, dada por:

$$
\begin{aligned}
\varphi: J \times[0,2 \pi[ & \longrightarrow \mathbb{R}^{3} \\
(t, \phi) & \longrightarrow(x(t) \cos \phi, x(t) \sin \phi, z(t)) .
\end{aligned}
$$

Em vista das condições (4.1) e (4.2), segue facilmente que $\varphi$ é uma imersão, sendo esta uma condição necessária para que a superfície $S$ possa ser um mergulho em $\mathbb{R}^{3}$. Com as hipóteses colocadas, podemos eventualmente obter, da classificação feita a seguir, superfícies que não sejam mergulhadas. No entanto, queremos que as soluções do nosso problema não tenham auto-intersecções e portanto, excluíremos aquelas que não nos interessam.

Por (4.3), o campo normal unitário $\mathrm{N}$ em $\mathrm{S}$ fica dado por:

$$
N(t, \phi)=\frac{1}{\sqrt{\dot{x}^{2}(t)+\dot{z}^{2}(t)}}(-\dot{z}(t) \cos \phi,-\dot{z}(t) \sin \phi, \dot{x}(t)) .
$$

Em relação à normal unitária $\mathrm{N}$, a curvatura média $\mathrm{H}$ de $\mathrm{S}$ é definida por $H=\frac{1}{2}\left(k_{1}+k_{2}\right)$, onde $k_{1}$ e $k_{2}$ são as curvaturas principais de $\mathrm{S}$. Calculando-se as curvaturas principais, utilizando-se (4.3) e $\mathrm{N}$, obtemos a expressão de $\mathrm{H}$, que é bem conhecida e é dada por (omitindo-se t )

$$
H=\frac{1}{2} \frac{1}{\sqrt{\dot{x}^{2}+\dot{z}^{2}}}\left(\frac{\dot{x} \ddot{z}-\ddot{x} \dot{z}}{\dot{x}^{2}+\dot{z}^{2}}+\frac{\dot{z}}{x}\right) .
$$




\subsection{Caracterização inicial da superfície rotacional $S$ a partir de sua} curvatura média $\boldsymbol{H}$

Lema 4.1.1. No nosso caso, em que a curvatura média $H$ é constante, podemos ainda simplificar (4.4) como

$$
\frac{\dot{z}}{\sqrt{\dot{x}^{2}+\dot{z}^{2}}}=H x+\frac{c}{x}
$$

onde $c$ é uma constante de integração.

Prova: Mostramos primeiramente que (4.4) é equivalente a

$$
\left(\frac{x \dot{z}}{\sqrt{\dot{x}^{2}+\dot{z}^{2}}}\right)=H\left(x^{2}\right) .
$$

Para isto, basta calcularmos que se $z=z(x)$ é uma solução qualquer de (4.4), então também satisfaz (4.6) e vice-versa. Então, integrando (4.6) obtemos:

$\frac{x \dot{z}}{\sqrt{\dot{x}^{2}+\dot{z}^{2}}}=H x^{2}+c$, onde c é uma constante de integração, e dividindo esta expressão por $x$, visto que $x>0$, obtemos (4.5). Ou seja, mostramos que (4.4) integração resulta em (4.5), com H e c constantes. Consequentemente (4.4) $\Longrightarrow(4.5)$.

Reciprocamente, usando a derivação de (4.5), mostramos que (4.5) $\Longrightarrow(4.4)$, no caso em que $\mathrm{H}$ e c são constantes.

\subsection{Caracterização inicial da superfície rotacional S a partir de sua curvatura média $H$}

Sejam $\gamma(t)=(x(t), 0, z(t))$ e S a superfície rotacional gerada por $\gamma$, introduzidas na seção 4.1 . O principal objetivo desta seção é mostrar que podemos determinar unicamente a curvatura média $\mathrm{H}$ de $\mathrm{S}$ no intervalo em que $x(t)$ varia.

Começamos observando que em vista da hipótese (4.1), podemos supor que $\gamma$ esteja parametrizada pelo comprimento de arco, ou seja,

$$
\|\dot{\gamma}(t)\|=\sqrt{\dot{x}^{2}(t)+\dot{z}^{2}(t)}=1, \quad \forall t \in J .
$$

Desta forma, (4.5) se torna

$$
\dot{z}=H x+\frac{c}{x}
$$


com H e c constantes. Por (4.7) temos $\dot{z}^{2}=1-\dot{x}^{2}$, e por (4.8) temos $\dot{z}^{2}=\left(H x+\frac{c}{x}\right)^{2}$, de onde segue que $\dot{x}^{2}=1-\left(H x+\frac{c}{x}\right)^{2}$. Ou seja,

$$
|\dot{x}|=\sqrt{1-\left(H x+\frac{c}{x}\right)^{2}} .
$$

Mas, (4.9) pode ser reescrita como $|\dot{x}|=\sqrt{\frac{x^{2}-\left(H x^{2}+c\right)^{2}}{x^{2}}} \mathrm{e}$, por (4.2), temos

$$
x|\dot{x}|=\sqrt{x^{2}-\left(H x^{2}+c\right)^{2}}=: \sqrt{P(x)} .
$$

Estamos interessados em, somente, $P(x) \geq 0$. Definimos:

$$
P_{+}:=\{x>0: P(x)>0\} \quad \text { e } \quad P_{0}:=\{x>0: P(x)=0\} .
$$

A seguir, vamos determinar explicitamente os conjuntos $P_{+}$e $P_{0}$ em função de $\mathrm{H}$ e c, com $\mathrm{H}$ e c como em (4.5).

Se $H=c=0$, então $P(x)=x^{2}$ e portanto, $\left.P_{+}=\right] 0, \infty\left[\right.$ e $P_{0}=\emptyset$.

Se $H \neq 0$ e $c=0$, então $P(x)=x^{2}-H^{2} x^{4}=x^{2}\left(1-H^{2} x^{2}\right)$. Neste caso, é fácil vermos que $\left.P_{+}=\right] 0,|H|^{-1}\left[\right.$ e $P_{0}=\left\{|H|^{-1}\right\}$.

Se $H=0$ e $c \neq 0$, então $P(x)=x^{2}-c^{2}$. Temos então que $\left.P_{+}=\right]|c|, \infty\left[\right.$ e $P_{0}=\{|c|\}$.

Como estamos interessados em determinar os zeros reais positivos de $P(x)$, então para o caso $H, c \neq 0$ e portanto, $P(x)=-H^{2} x^{4}+(1-2 H c) x^{2}-c^{2}$, devemos ainda supor $4 H c \leq 1$. Com esta hipótese, é fácil mostrarmos que $1-2 H c \pm \sqrt{1-4 H c}$ são positivos e, assim, temos os zeros reais positivos de $P(x)$ :

$$
\xi_{1}=\sqrt{\frac{1-2 H c-\sqrt{1-4 H c}}{2 H^{2}}}, \quad \xi_{2}=\sqrt{\frac{1-2 H c+\sqrt{1-4 H c}}{2 H^{2}}} .
$$

Neste caso, calculando $P(0)=-c^{2}$, concluímos que $\left.P_{+}=\right] \xi_{1}, \xi_{2}\left[\right.$ e $P_{0}=\left\{\xi_{1}, \xi_{2}\right\}$.

Resumindo estas informações na seguinte tabela, que denotamos por $(T)$ : 


\subsection{Caracterização inicial da superfície rotacional $S$ a partir de sua}

curvatura média $H$

\begin{tabular}{|r|r|c|c|}
\hline$H$ & $c$ & $\Rightarrow P_{+}$ & $P_{0}$ \\
\hline 0 & 0 & ] $0, \infty[$ & $\emptyset$ \\
\hline$\neq 0$ & 0 & ] $0,|H|^{-1}[$ & $\left\{|H|^{-1}\right\}$ \\
\hline 0 & $\neq 0$ & ]$|c|, \infty[$ & $\{|c|\}$ \\
\hline$\neq 0$ & $\neq 0$ & ]$\xi_{1}, \xi_{2}[$ & $\left\{\xi_{1}, \xi_{2}\right\}$ \\
\hline
\end{tabular}

Considerando as definições e resultados obtidos até agora nesta seção 4.2, apresentamos o seguinte resultado.

Lema 4.2.1. Sejam $\gamma(t)=(x(t), 0, z(t)), \gamma$ parametrizada pelo comprimento de arco e $S$ a superfície de revolução gerada por $\gamma$, com as hipóteses para $\gamma$ introduzidas na seção 4.1 . Sejam $H$ e c constantes, como em (4.5). Definimos: $\rho_{1}:=\inf _{t \in J} x(t)$ e $\rho_{2}:=\sup _{t \in J} x(t)$. Então, $\left.P_{+}=\right] \rho_{1}, \rho_{2}[$.

Prova: Por (4.2), $x(t)>0, \forall t \in J$ e portanto, há somente as 4 possibilidades seguintes para examinarmos, com base na informação de que $x(t)$ é uma solução das equações diferenciais (4.9) e (4.10).

( $1 \stackrel{a}{ }) \rho_{1}=0$.

Fazendo $x \rightarrow \rho_{1}=0$ em (4.9), vemos que é necessário tomar $c=0$; caso contrário, obteríamos um valor negativo dentro da raiz. Além disto, no caso em que $c=0$ temos pela tabela $(T)$ que $\left.P_{+}=\right] 0, \infty\left[\right.$ ou $\left.P_{+}=\right] 0,|H|^{-1}\left[\right.$, ou seja, $\left.P_{+}=\right] \rho_{1}, \infty\left[\right.$ ou $\left.P_{+}=\right] \rho_{1},|H|^{-1}[$.

( $\left.2^{\underline{a}}\right) \rho_{2}=\infty$.

Fazendo $x \rightarrow \rho_{2}=\infty$ em (4.9), vemos que é necessário tomar $H=0$; caso contrário, obteríamos um valor negativo dentro da raiz. Além disto, no caso em que $H=0$, temos pela tabela $(T)$ que $\left.P_{+}=\right] 0, \infty\left[\right.$ ou $\left.P_{+}=\right]|c|, \infty\left[\right.$, ou seja, $\left.P_{+}=\right] 0, \rho_{2}\left[\right.$ ou $\left.P_{+}=\right]|c|, \rho_{2}[$.

( $\left.3^{\underline{a}}\right) \rho_{1}>0$.

Tomamos $J=$ ] $t_{1}, t_{2}$ [ como o máximo intervalo de integração da equação diferencial (4.10). Podemos dividir a análise em 2 casos:

(a) Suponhamos, sem perda de generalidade, que $x(t)$ seja crescente em $J=] t_{1}, t_{2}[$. 
Como a solução $x(t)$ é contínua, visto que $\gamma(t)=(x(t), 0, z(t)) \in C^{2}\left(J, \mathbb{R}^{3}\right)$ e $\rho_{1}=\inf _{t \in J} x(t)>$ 0 , então $\rho_{1}=\lim _{t \rightarrow t_{1}} x(t)$.

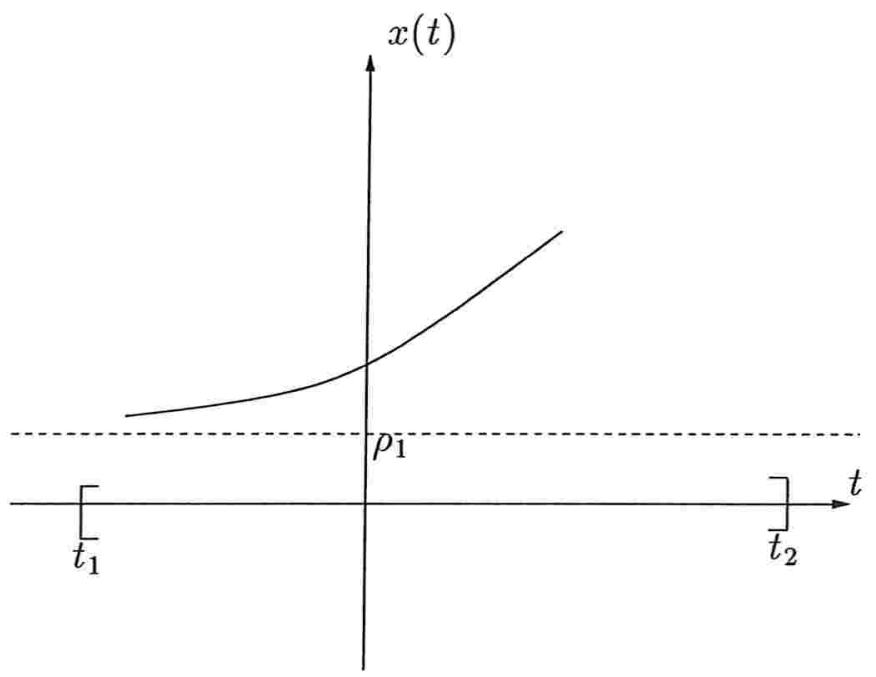

Figura 4.1: Representação do gráfico da função $y=x(t)$

Com isto, temos dois subcasos de (a) para estudar:

(i) $t_{1}=-\infty$.

Neste caso, $x(t)=\rho_{1}$ é uma assíntota horizontal do gráfico de $x(t)$. Com isto,

$$
\lim _{t \rightarrow t_{1}=-\infty} x(t)=\rho_{1} \quad \text { e } \quad \lim _{t \rightarrow t_{1}=-\infty} \dot{x}(t)=0 .
$$

Logo, calculando $\lim _{t \rightarrow t_{1}}$ em (4.10) temos:

$\lim _{t \rightarrow t_{1}}|\dot{x}(t)| x(t)=\lim _{t \rightarrow t_{1}} \sqrt{P(x)}$. Pela continuidade das funções $\dot{x}(t), x(t)$, do módulo, da raiz quadrada e de $P(x)$, segue:

$\left|\lim _{t \rightarrow t_{1}} \dot{x}(t)\right| \lim _{t \rightarrow t_{1}} x(t)=\sqrt{P\left(\lim _{t \rightarrow t_{1}} x(t)\right)}$. Por (4.12), temos:

$$
P\left(\rho_{1}\right)=0 \text {. }
$$

(ii) $t_{1}>-\infty$.

Sabemos, por definição, que $P(x) \geq 0$. Suponha, por absurdo, que $P\left(\rho_{1}\right)>0$. Neste caso (a), supomos $x(t)$ crescente e portanto, $\dot{x}(t)>0, \forall t \in J$. Com isto (4.10) pode ser reescrita como

$$
\dot{x}=\frac{\sqrt{P(x)}}{x} \text { em } J .
$$




\subsection{Caracterização inicial da superfície rotacional $S$ a partir de sua} curvatura média $H$

Como $P\left(\rho_{1}\right)>0$ e $P(x)$ é contínua em $\mathrm{J}$, segue que $P(x)>0$ em uma vizinhança de $\rho_{1}$. Da mesma forma, como $\rho_{1}>0$ então quando tomamos o $\lim _{t \rightarrow t_{1}}$ em (4.13), o denominador é ainda não-nulo. Concluímos então que a equação diferencial (4.13) pode ser resolvida não somente para $t=t_{1}$, mas também em uma vizinhança dele, o que contradiz o fato de $J=] t_{1}, t_{2}$ [ ser o máximo intervalo de integração. Consequentemente,

$$
P\left(\rho_{1}\right)=0
$$

(b) Suponhamos que $x$ não seja monótono, isto é, $\left.\exists t_{0} \in\right] t_{1}, t_{2}$ [ tal que $\dot{x}\left(t_{0}\right)=0$. Por (4.10), temos que:

$$
\left|\dot{x}\left(t_{0}\right)\right| x\left(t_{0}\right)=\sqrt{P\left(x\left(t_{0}\right)\right)} \text {, e então }
$$

$$
P\left(x\left(t_{0}\right)\right)=0 \text {. }
$$

Pela tabela $(T)$ podemos ver que os zeros de $P(x)$ pertencem aos extremos do intervalo $P_{+}$. Desta forma, segue de $(T)$ e (4.14) que:

$$
x\left(t_{0}\right)=|H|^{-1},|c|, \xi_{1} \text { ou } \xi_{2} .
$$

Suponhamos que $x\left(t_{0}\right)=|c|$ ou $\xi_{1}$. Estudando o gráfico de $P(x)$ nos respectivos casos, concluímos que:

- no caso $H=0, c \neq 0:|c|=\lim _{t \rightarrow t_{1}} x(t)=\rho_{1}$,

- no caso $H, c \neq 0: \xi_{1}=\lim _{t \rightarrow t_{1}} x(t)=\rho_{1}$.

Suponhamos analogamente que $x\left(t_{0}\right)=|H|^{-1}$ ou $\xi_{2}$. Estudando o gráfico de $P(x)$, temos:

- no caso $H \neq 0, c=0:|H|^{-1}=\lim _{t \rightarrow t_{2}} x(t)=\rho_{2}$,

- no caso $H, c \neq 0: \xi_{2}=\lim _{t \rightarrow t_{2}} x(t)=\rho_{2}$.

Com isto, mostramos que $x\left(t_{0}\right)=\rho_{1}$ ou $\rho_{2}$,ou seja, mostramos que se existe $\left.t_{0} \in\right] t_{1}, t_{2}[$ com $\dot{x}\left(t_{0}\right)=0$ então $x\left(t_{0}\right)$ só pode ser o ínfimo ou o supremo de $x(t)$. Em razão disto, podemos trabalhar com $x(t)$ como sendo monótono e portanto, recaímos no caso (a) estudado. Logo, $P\left(\rho_{1}\right)=0$.

Com isto, mostramos que se $\rho_{1}>0$ então $P\left(\rho_{1}\right)=0$. Ou seja, $\rho_{1} \in P_{0}$. Como $\rho_{1}=\inf _{t \in J} x(t)$, então $\rho_{1}=|c|$ ou $\rho_{1}=\xi_{1}$. No caso em que $\rho_{1}=|c|$, temos pela tabela $(T)$ 
que $\left.P_{+}=\right]|c|, \infty\left[\right.$. Se $\rho_{1}=\xi_{1}$, pela tabela $(T)$ temos $\left.P_{+}=\right] \xi_{1}, \xi_{2}[$. Isto é, mostramos para a $\left(3^{\underline{a}}\right)$ possibilidade que $\left.P_{+}=\right] \rho_{1}, \infty\left[\right.$ ou $\left.P_{+}=\right] \rho_{1}, \xi_{2}[$.

(4⿳亠丷a $) \rho_{2}<\infty$.

Novamente tomamos $J=] t_{1}, t_{2}$ [ o máximo intervalo de integração da equação diferencial (4.10). Procedendo exatamente como na $\left(3^{\underline{a}}\right)$ possibilidade, mas agora considerando o extremo $t_{2}$, quando $t_{2}=\infty$ ou $t_{2}<\infty$ concluímos que $P\left(\rho_{2}\right)=0$.

Ou seja, se $\rho_{2}<\infty$ então $P\left(\rho_{2}\right)=0$, isto é, $\rho_{2} \in P_{0}$. Como $\rho_{2}=\sup _{t \in J} x(t)$, então $\rho_{2}=|H|^{-1}$ ou $\rho_{2}=\xi_{2}$. No caso em que $\rho_{2}=|H|^{-1}$, temos pela tabela $(T)$ que $\left.P_{+}=\right] 0,|H|^{-1}\left[\right.$. Se $\rho_{2}=\xi_{2}$, pela tabela $(T)$ temos $\left.P_{+}=\right] \xi_{1}, \xi_{2}$ [. Isto é, mostramos para a $\left(4^{\underline{a}}\right)$ possibilidade que $\left.P_{+}=\right] 0, \rho_{2}\left[\right.$ ou $\left.P_{+}=\right] \xi_{1}, \rho_{2}[$.

Juntando as informações obtidas, vemos que estes 4 casos cobrem todas as possibilidades da tabela $(T)$ e portanto, podemos concluir que $\left.P_{+}=\right] \rho_{1}, \rho_{2}[$.

Teorema 4.2.2. Sejam $\gamma(t)=(x(t), 0, z(t)), x(t)>0, \gamma \in C^{2}\left(J, \mathbb{R}^{3}\right), J \subset \mathbb{R}$ um intervalo aberto, uma curva regular parametrizada pelo comprimento de arco e $S$ a superfície de revolução, parametrizada por (4.3), gerada pela revolução da curva $\gamma$ ao redor do eixo z. Sejam H e c constantes, como em (4.5). Então podem ocorrer exatamente as cinco seguintes possibilidades para o valor da curvatura média $H$ de $S$, em função de $\rho_{1}$ e $\rho_{2}$ (definidos no Lema 4.2.1):

(1) Se $\rho_{1}=0$ e $\rho_{2}=\infty$, então

$$
H=c=0
$$

e $z(t)$ é constante em. $J$.

(2) Se $\rho_{1}=0$ e $\rho_{2}<\infty$, então $\dot{z}(t) \neq 0, \forall t \in J, e$

$$
H=\frac{1}{\rho_{2}} \operatorname{sgn} \dot{z}, c=0
$$

(onde sgn denota o sinal de uma função) e existe $\beta \in \mathbb{R}$ com $x^{2}+(z-\beta)^{2}=\rho_{2}^{2}$ em J.

(3) $S e \rho_{1}>0$ e $\rho_{2}=\infty$, então

$$
H=0,|c|=\rho_{1}
$$




\subsection{Caracterização inicial da superfície rotacional $S$ a partir de sua} curvatura média $H$

e existe $\beta \in \mathbb{R}$ com $x=\rho_{1} \cosh \frac{z-\beta}{\rho_{1}}$ em $J$.

(4) Se $\rho_{1}>0, \rho_{2}<\infty$ e, além disto, $\dot{z}(t) \neq 0, \forall t \in J$, então

$$
H=\frac{1}{\rho_{1}+\rho_{2}} \operatorname{sgn} \dot{z}, \quad c=\frac{\rho_{1} \rho_{2}}{\rho_{1}+\rho_{2}} \operatorname{sgn} \dot{z} .
$$

(5) Se $\rho_{1}>0, \rho_{2}<\infty$ e $\dot{z}\left(t_{0}\right)=0$ para, no minimo, um $t_{0} \in J$, então

$$
H=-\frac{1}{\rho_{2}-\rho_{1}} \operatorname{sgn} \dot{z}\left(t_{1}\right), \quad c=\frac{\rho_{1} \rho_{2}}{\rho_{2}-\rho_{1}} \operatorname{sgn} \dot{z}\left(t_{1}\right),
$$

onde $t_{1} \in J \operatorname{com} x\left(t_{1}\right)=\rho_{1}$.

Prova: No Lema 4.2.1, provamos que o conjunto $P_{+}$, definido no início da seção 4.2, é tal que $\left.P_{+}=\right] \rho_{1}, \rho_{2}$ [. Com isto, passamos a estudar cada um dos cinco casos:

(1) $\rho_{1}=0$ e $\rho_{2}=\infty$, isto é, $\left.P_{+}=\right] 0, \infty[$. Pela tabela $(T)$, temos que para este caso é necessário $H=c=0$.

Por (4.5) segue que $\dot{z}(t)=0, \forall t \in J$, ou seja, $z(t)$ é constante em J.

Podemos então concluir que a superfície de revolução obtida neste caso é um plano, paralelo ao plano coordenado $0 x y$.

(2) $\rho_{1}=0$ e $\rho_{2}<\infty$, isto é, $\left.P_{+}=\right] 0, \rho_{2}[$. Pela tabela $(T)$, temos então que

$$
c=0, \rho_{2}=|H|^{-1} \text {. }
$$

Logo, por (4.5) temos $\dot{z}=H x$; e por (4.10) temos $|\dot{x}| x=\sqrt{x^{2}-H^{2} x^{4}}=$ $x \sqrt{1-H^{2} x^{2}}$, ou seja, $|\dot{x}|=\sqrt{1-H^{2} x^{2}}$.

Juntando estas últimas informações, obtemos:

$$
\begin{aligned}
\dot{z} & =H x=H x \frac{|\dot{x}|}{|\dot{x}|}=\frac{H x|\dot{x}|}{\sqrt{1-H^{2} x^{2}}}=\frac{H \dot{x}}{H \dot{x}} \frac{H x|\dot{x}|}{\sqrt{1-H^{2} x^{2}}}=\frac{|\dot{x}|}{\dot{x}} \frac{1}{H} \frac{H^{2} x \dot{x}}{\sqrt{1-H^{2} x^{2}}} \\
& =-\frac{\operatorname{sgn} \dot{x}}{H}\left(\sqrt{1-H^{2} x^{2}}\right) .
\end{aligned}
$$

Integrando esta última expressão, obtemos:

$$
z(t)=-\frac{\operatorname{sgn} \dot{x}}{H} \sqrt{1-H^{2} x^{2}}+\beta
$$


onde $\beta$ é uma constante de integração. Além disto, por (4.16) e (4.15) temos:

$$
x^{2}+(z-\beta)^{2}=x^{2}+\frac{1}{H^{2}}\left(1-H^{2} x^{2}\right)=\frac{1}{H^{2}}=\rho_{2}^{2},
$$

que é a equação de um semicírculo (visto que $x>0$ ). Portanto, obtemos neste caso uma esfera como superfície de revolução.

Agora, por (4.15) e (4.5), temos:

$$
|\dot{z}|=|H||x|=|H| x .
$$

Então $|\dot{z}| \neq 0$, visto que $x>0$ e $|H|>0$, pois se $|H|=0$, então $\rho_{2}=\frac{1}{|H|}=\infty$, o que é absurdo neste caso. Novamente por (4.5) e (4.18), temos:

$$
\frac{\dot{z}}{|\dot{z}|}=\frac{H x}{|\dot{z}|}, \text { ou seja, } \operatorname{sgn} \dot{z}=\frac{H x}{|H| x} \text {. }
$$

Logo, por (4.15),

$$
H=|H| \operatorname{sgn} \dot{z}=\frac{1}{\rho_{2}} \operatorname{sgn} \dot{z}, \text { para } t \in J \text {. }
$$

(3) $\rho_{1}>0$ e $\rho_{2}=\infty$, isto é, $\left.P_{+}=\right] \rho_{1}, \infty[$. Pela tabela $(T)$ obtemos neste caso

$$
H=0 \quad \text { e } \quad \rho_{1}=|c| .
$$

Logo, por (4.5) temos $\dot{z}=\frac{c}{x}$; e por (4.10) temos $|\dot{x}| x=\sqrt{x^{2}-c^{2}}$, ou seja, $x=\frac{\sqrt{x^{2}-c^{2}}}{|\dot{x}|}$, visto que $|\dot{x}| \neq 0, \forall t \in J$; caso contrário, teríamos $x(t)=$ constante e portanto, $\rho_{1}=\rho_{2}=\infty$, o que seria absurdo neste caso, pois queremos $\rho_{1}<\infty$. Juntando estas últimas informações, resulta que:

$$
\begin{aligned}
\dot{z} & =\frac{c}{x}=\frac{c|\dot{x}|}{\sqrt{x^{2}-c^{2}}}=\frac{c|\dot{x}|}{\sqrt{c^{2}\left(\frac{x^{2}}{c^{2}}-1\right)}} \frac{\dot{x}}{\dot{x}}=c \operatorname{sgn} \dot{x} \frac{\dot{x}}{|c| \sqrt{\left(\frac{x}{|c|}\right)^{2}-1}} \\
& =c \operatorname{sgn} \dot{x}\left(\operatorname{arcosh} \frac{x}{|c|}\right) .
\end{aligned}
$$

Integrando esta última expressão obtemos:

$$
z(t)=c \operatorname{sgn} \dot{x}\left(\operatorname{arcosh} \frac{x}{|c|}\right)+\beta
$$




\subsection{Caracterização inicial da superfície rotacional $S$ a partir de sua curvatura média $H$}

onde $\beta$ é uma constante de integração e $t \in J$. Observamos que $x(t) \geq \rho_{1}=|c|$ e portanto, $\frac{x(t)}{|c|} \geq 1 ;$ logo, faz sentido falar em $\operatorname{arcosh} \frac{x(t)}{|c|}$.

De (4.20) e (4.19) segue:

$|z(t)-\beta|=|c||\operatorname{sgn} \dot{x}|\left|\operatorname{arcosh} \frac{x(t)}{|c|}\right|=\rho_{1} \operatorname{arcosh} \frac{x(t)}{\rho_{1}}$, visto que Im arcosh $=[0, \infty[$. Consequentemente,

$\cosh \left(\frac{|z(t)-\beta|}{\rho_{1}}\right)=\frac{x(t)}{\rho_{1}}$, e isto implica que, $\forall t \in J, \quad x(t)=\rho_{1} \cosh \left(\frac{z(t)-\beta}{\rho_{1}}\right)$, pois cosh é uma função par.

A equação acima descreve uma curva denominada catenária, cuja superfície de revolução chamamos de catenóide.

Antes de estudarmos especificamente os casos (4) e (5), vamos obter expressões gerais para $\mathrm{H}$ e c quando $\rho_{1}>0$ e $\rho_{2}<\infty$.

Pela tabela $(T)$ temos que, neste caso, $H, c \neq 0, \rho_{1}=\xi_{1}, \rho_{2}=\xi_{2}$. Por (4.11) temos: $\left(\rho_{2} \pm \rho_{1}\right)^{2}=\left(\xi_{2} \pm \xi_{1}\right)^{2}=\xi_{2}^{2}+\xi_{1}^{2} \pm 2 \xi_{1} \xi_{2}=\frac{1-2 H c \pm 2|H c|}{H^{2}}$, ou seja,

$$
H^{2}\left(\rho_{2} \pm \rho_{1}\right)^{2}=1-2 H c \stackrel{ \pm}{-}|H c| .
$$

Também temos que: $\rho_{1} \rho_{2}=\xi_{1} \xi_{2}=\frac{2|H c|}{2 H^{2}}=\left|\frac{c}{H}\right|$, ou seja,

$$
|c|=|H| \rho_{1} \cdot \rho_{2} \text {. }
$$

- Se $H c>0$ então por (4.21): $H^{2}\left(\rho_{2}+\rho_{1}\right)^{2}=1$ e $H^{2}\left(\rho_{2}-\rho_{1}\right)^{2}=1-4 H c$. Como $\rho_{1}+\rho_{2}>0$, segue da primeira equação que $\rho_{1}+\rho_{2}=|H|^{-1}$. Da segunda equação segue que $\left(\rho_{2}-\rho_{1}\right)^{2}=\frac{1}{H^{2}}-4 \frac{c}{H}$. Mas, por (4.22), temos que para o caso $H c>0$, vale $\frac{c}{H}=\rho_{1} \rho_{2}$. Logo, $\left(\rho_{2}-\rho_{1}\right)^{2}=\frac{1}{H^{2}}-4 \rho_{1} \rho_{2}$, ou seja, $\left(\rho_{2}+\rho_{1}\right)^{2}=\frac{1}{H^{2}}$. Portanto, da segunda equação também segue que $\rho_{1}+\rho_{2}=|H|^{-1}$.

- Se $H c<0$ então mostramos de forma análoga ao que foi feito acima que vale $\rho_{2}-\rho_{1}=|H|^{-1}$.

Resumindo estas informações:

$$
\begin{aligned}
& |H|^{-1}=\rho_{1}+\rho_{2}, \quad \text { se } H c>0 ; \\
& |H|^{-1}=\rho_{2}-\rho_{1}, \text { se } H c<0 .
\end{aligned}
$$


Passemos a estudar os casos (4) e (5).

(4) $\rho_{1}>0, \rho_{2}<\infty$, isto é, $\left.P_{+}=\right] \rho_{1}, \rho_{2}\left[\right.$ e $\dot{z}(t) \neq 0, \forall t \in J$. Como $\rho_{2} \geq \rho_{1}$, podemos dividir em dois subcasos:

(i) $\rho_{1}=\rho_{2}$, isto é, $x(t)$ é constante, e isto implica que $\dot{x}(t)=0, \forall t \in J$. Por (4.4) temos $2 H=\frac{\dot{z}}{x}$. Usando que $\dot{x}(t)=0, \forall t \in J$, e $\gamma$ está parametrizada pelo comprimento de arco, segue que $\dot{z}= \pm 1$, ou seja, $|\dot{z}|=1$. Juntando estas últimas informações, temos:

$$
2 H=\frac{\dot{z}}{x}=\frac{1}{x} \frac{\dot{z}}{|\dot{z}|}=\frac{1}{x} \operatorname{sgn} \dot{z} \quad \text { em } \quad J
$$

Consequentemente,

$$
\begin{aligned}
& 2|H|=\frac{1}{|x|}|\operatorname{sgn} \dot{z}| \text { e como } x>0 \text { segue que } \\
& \qquad 2|H|=\frac{1}{x} .
\end{aligned}
$$

Por (4.5), (4.26) e (4.25) temos que

$$
\begin{aligned}
\frac{\dot{z}}{|\dot{z}|}=H x+\frac{c}{x}, \text { ou seja, sgn } \dot{z} & =\frac{1}{2} \operatorname{sgn} \dot{z}+2|H| c \text {. Portanto, } \\
c & =\frac{1}{4|H|} \operatorname{sgn} \dot{z} \quad \text { em } \quad J .
\end{aligned}
$$

Observamos que se $H>0$, então por (4.25) teríamos sgn $\dot{z}>0$ e por (4.27) teríamos $c>0$. Se $H<0$, então por (4.25) teríamos sgn $\dot{z}<0$ e por (4.27) teríamos $c<0$. Concluímos que para este caso deve valer $H c>0$.

Portanto, no caso $\rho_{1}=\rho_{2}$, obtemos de (4.23) que $|H|^{-1}=\rho_{1}+\rho_{2}=2 \rho_{1}$.

Novamente por (4.25) e (4.26), obtemos: $2 H=\frac{1}{x} \operatorname{sgn} \dot{z}=2|H| \operatorname{sgn} \dot{z}=2 \cdot \frac{1}{2 \rho_{1}} \operatorname{sgn} \dot{z}$, ou seja,

$$
H=\frac{1}{2 \rho_{1}} \operatorname{sgn} \dot{z}
$$

De (4.28) e (4.27) segue que :

$$
c=\frac{1}{4} 2 \rho_{1} \operatorname{sgn} \dot{z}=\frac{\rho_{1}}{2} \operatorname{sgn} \dot{z} .
$$

Conforme já observamos, temos, neste caso, $x(t)=$ constante em J. Portanto, obtemos um cilindro como superfície de revolução quando $\rho_{1}=\rho_{2}$. 


\subsection{Caracterização inicial da superfície rotacional $S$ a partir de sua} curvatura média $H$

Lembramos que quando $H, c \neq 0$, havíamos suposto que $4 H c \leq 1$. Devemos mostrar então que, com os resultados obtidos neste caso, a hipótese acima está satisfeita. De fato, temos por (4.28) e (4.29) que

$$
4 H c=4 \frac{1}{2 \rho_{1}} \operatorname{sgn} \dot{z} \frac{\rho_{1}}{2} \operatorname{sgn} \dot{z}=1 .
$$

(ii) $\rho_{1}<\rho_{2}$. Mostramos a seguir que neste caso também deve valer $H c>0$. Suponha por absurdo, que $H c<0$. Logo, $-\frac{c}{H}>0$ e portanto, para o particular $x=\sqrt{-\frac{c}{H}}$ deve valer que:

$P(x):=x^{2}-\left(H x^{2}+c\right)^{2}=-\frac{c}{H}-\left(H\left(-\frac{c}{H}\right)+c\right)^{2}=-\frac{c}{H}>0$. Desta forma, pela definição de $P_{+}$, segue que $\left.x=\sqrt{-\frac{c}{H}} \in P_{+}=\right] \rho_{1}, \rho_{2}\left[\right.$. Podemos reescrever (4.5) como $\dot{z} x=H x^{2}+c$ e portanto, para o particular $x=\sqrt{-\frac{c}{H}} \in P_{+}$temos:

$\dot{z} \sqrt{-\frac{c}{H}}=H\left(-\frac{c}{H}\right)+c=0$, de onde segue que $\dot{z}=0$, o que está em contradição com a hipótese de que $\dot{z}(t) \neq 0, \forall t \in J$. Portanto, $H c>0$.

Multiplicando (4.5) por $\mathrm{H}$ obtemos $H \dot{z}=H^{2} x+\frac{H c}{x}$. Como $H^{2} x$ e $\frac{H c}{x}$ são positivos, então H e $\dot{z}$ têm o mesmo sinal. Portanto,

$$
|H|^{-1}=\frac{1}{|H|}=\frac{1}{H} \operatorname{sgn} H=\frac{1}{H} \operatorname{sgn} \dot{z} .
$$

Por (4.23) e (4.30) temos

$$
\rho_{1}+\rho_{2}=\frac{1}{H} \operatorname{sgn} \dot{z}, \text { ou seja, } H=\frac{1}{\rho_{1}+\rho_{2}} \operatorname{sgn} \dot{z} .
$$

Como $H c>0$, temos por (4.22) que:

$$
c=H \rho_{1} \rho_{2}==\frac{\rho_{1} \rho_{2}}{\rho_{1}+\rho_{2}} \operatorname{sgn} \dot{z} .
$$

Veremos, na seção 4.4, que este caso corresponde à trajetória de um dos focos de uma elipse.

Devemos mostrar também aqui que vale $4 H c \leq 1$. De fato, por (4.31) e (4.32) temos: $4 H c=4 \frac{\rho_{1} \rho_{2}}{\left(\rho_{1}+\rho_{2}\right)^{2}}$. Basta então mostrar que $\frac{\rho_{1} \rho_{2}}{\left(\rho_{1}+\rho_{2}\right)^{2}} \leq \frac{1}{4}$. Suponha, por absurdo, 
que $\frac{\rho_{1} \rho_{2}}{\left(\rho_{1}+\rho_{2}\right)^{2}}>\frac{1}{4}$, ou seja, $4 \rho_{1} \rho_{2}>\left(\rho_{1}+\rho_{2}\right)^{2}=\rho_{1}^{2}+\rho_{2}^{2}+2 \rho_{1} \rho_{2}$. Seguirá disto que: $\rho_{1}^{2}-2 \rho_{1} \rho_{2}+\rho_{2}^{2}<0$, isto é, $\left(\rho_{1}-\rho_{2}\right)^{2}<0$, o que é absurdo.

(5) $\rho_{1}>0$ e $\rho_{2}<\infty$, isto é, $\left.P_{+}=\right] \rho_{1}, \rho_{2}\left[\right.$ e $\dot{z}\left(t_{0}\right)=0$ para, no mínimo, um $t_{0} \in J$.

Observamos inicialmente que $\rho_{1}=\rho_{2}$ não ocorre neste caso. Caso contrário, teríamos $\dot{x}(t)=0, \forall t \in J$, e portanto, $\left\|\dot{\gamma}\left(t_{0}\right)\right\|=\sqrt{\dot{x}^{2}\left(t_{0}\right)+\dot{z}^{2}\left(t_{0}\right)}=0$, o que contraria a hipótese da regularidade de $\gamma$.

Podemos reescrever (4.5) como $\dot{z} x=H x^{2}+c$ e, consequentemente, por hipótese,

$$
H x^{2}\left(t_{0}\right)+c=\dot{z}\left(t_{0}\right) x\left(t_{0}\right)=0 .
$$

Disto segue que as constantes $\mathrm{H}$ e c são tais que $H c<0$; caso contrário, multiplicando a expressão por c, teríamos: $H c x^{2}\left(t_{0}\right)+c^{2}=0$, e isto implicaria que $c=0$, o que é absurdo para este caso.

Por definição, temos $\rho_{1}=\inf _{t \in J} x(t)=\lim _{t \rightarrow t_{1}} x(t)$ e portanto, $0<\rho_{1}<x\left(t_{0}\right)$. Logo, $\rho_{1}^{2}<x^{2}\left(t_{0}\right)$. Somando $\frac{c}{H}$ nesta desigualdade, obtemos de (4.33):

$$
\rho_{1}^{2}+\frac{c}{H}<x^{2}\left(t_{0}\right)+\frac{c}{H}=\frac{H x^{2}\left(t_{0}\right)+c}{H}=0 .
$$

Ou seja,

$$
\rho_{1}^{2}+\frac{c}{H}<0
$$

Denotando por $\dot{z}\left(t_{1}\right)$ o $\lim _{t \rightarrow t_{1}} \dot{z}(t)$ e calculando $\lim _{t \rightarrow t_{1}}$ em (4.5), temos:

$$
\dot{z}\left(t_{1}\right)=H \rho_{1}+\frac{c}{\rho_{1}}, \quad \text { ou seja }, \quad \dot{z}\left(t_{1}\right) \rho_{1}=H \rho_{1}^{2}+c .
$$

Denotando por $\dot{x}\left(t_{1}\right)$ o $\lim _{t \rightarrow t_{1}} \dot{x}(t)$, como $\gamma$ está parametrizada pelo comprimento de arco, então

$$
\dot{x}^{2}\left(t_{1}\right)+\dot{z}^{2}\left(t_{1}\right)=1
$$

Calculando $\lim _{t \rightarrow t_{1}}$ na expressão de $P(x)$, obtemos por (4.35) e (4.36)

$$
P\left(\rho_{1}\right)=\rho_{1}^{2}-\left(H \rho_{1}^{2}+c\right)^{2}=\rho_{1}^{2}-\left(\dot{z}\left(t_{1}\right) \rho_{1}\right)^{2}=\rho_{1}^{2} \cdot \dot{x}^{2}\left(t_{1}\right)
$$




\subsection{Caracterização inicial da superfície rotacional $S$ a partir de sua curvatura média $H$}

No entanto, mostramos com a $3 \stackrel{a}{a}$ possibilidade do Lema 4.2 .1 que se $\rho_{1}>0$ então $P\left(\rho_{1}\right)=0$. Logo, $\rho_{1}^{2} \dot{x}^{2}\left(t_{1}\right)=0$, de onde segue que $\dot{x}\left(t_{1}\right)=0$, pois $\rho_{1}>0$. Por (4.36) temos que $\dot{z}^{2}\left(t_{1}\right)=1$, isto é, $\left|\dot{z}\left(t_{1}\right)\right|=1$.

Então, concluímos por (4.35) e (4.34) que

$$
\frac{1}{H} \rho_{1} \operatorname{sgn} \dot{z}\left(t_{1}\right)=\frac{1}{H} \rho_{1} \frac{\dot{z}\left(t_{1}\right)}{\left|\dot{z}\left(t_{1}\right)\right|}=\frac{1}{H} \rho_{1}\left(H \rho_{1}+\frac{c}{\rho_{1}}\right)=\rho_{1}^{2}+\frac{c}{H}<0 .
$$

Logo, $\frac{1}{H} \dot{z}\left(t_{1}\right)<0$, ou seja, H e $\dot{z}\left(t_{1}\right)$ têm sinais opostos. Então,

$$
|H|^{-1}=\frac{1}{|H|}=\frac{\operatorname{sgn} H}{H}=-\frac{1}{H} \operatorname{sgn} \dot{z}\left(t_{1}\right) .
$$

Como para este caso (5) temos $H c<0$, então por (4.24) e (4.37) temos

$$
\rho_{2}-\rho_{1}=-\frac{1}{H} \operatorname{sgn} \dot{z}\left(t_{1}\right), \quad \text { ou seja, } \quad H=-\frac{1}{\rho_{2}-\rho_{1}} \operatorname{sgn} \dot{z}\left(t_{1}\right) \text {. }
$$

Como $H c<0$, temos por (4.22) que

$$
c=-H \rho_{1} \rho_{2}=\frac{\rho_{1} \rho_{2}}{\rho_{2}-\rho_{1}} \operatorname{sgn} \dot{z}\left(t_{1}\right) .
$$

Veremos, na seção 4.4, que este caso corresponde à trajetória de um dos focos de uma hipérbole.

Devemos mostrar também que $4 H c \leq 1$. De fato, por (4.38) e (4.39) temos $4 H c=-\frac{4 \rho_{1} \rho_{2}}{\left(\rho_{2}-\rho_{1}\right)^{2}}$. Basta então mostrar que $\frac{\rho_{1} \rho_{2}}{\left(\rho_{2}-\rho_{1}\right)^{2}} \geq-\frac{1}{4}$. Mas, $\frac{\rho_{1} \rho_{2}}{\left(\rho_{2}-\rho_{1}\right)^{2}}>0>$ $-\frac{1}{4}$, visto que $\rho_{1} \rho_{2}>0$.

No Teorema 4.2.2 vimos que as curvas $\gamma$, nos casos (1) e (2), descrevem as equações de uma semi-reta paralela ao plano $0 x y$ e de um semicírculo, cujas superfícies de revolução são, respectivamente, um plano e uma esfera. No entanto, para as curvas $\gamma$ dos casos (3), (4) e (5), vamos mostrar que elas são geradas pela trajetória do foco de uma quádrica Q, não-degenerada, quando esta rola, sem deslizar, sobre o eixo $0 z$. Ou seja, mostraremos que as superfícies de revolução geradas pelas curvas $\gamma$ dos casos (3), (4) e (5) do Teorema 4.2 .2 correspondem exatamente às Superfícies de Delaunay.

Antes disto, porém, determinaremos um referencial móvel sobre a quádrica $Q$ e obteremos as coordenadas da trajetória do foco de Q, em função deste sistema. 


\subsection{Determinação de um referencial móvel sobre uma quádrica $\mathrm{Q}$}

Seja Q uma quádrica não-degenerada, ou seja, Q é uma parábola, uma elipse ou uma hipérbole, e F um de seus focos. Seja $X: J \rightarrow Q, J \subset \mathbb{R}$ um intervalo aberto, uma parametrização de Q. Definimos a função comprimento de arco de Q a partir de um $t_{0} \in J$, fixado, por

$$
s(t)=\int_{t_{0}}^{t}\|\dot{X}(\tau)\| d \tau .
$$

Sejam $e_{1}(t):=\frac{\dot{X}(t)}{\|\dot{X}(t)\|}$ o vetor tangente unitário a $\mathrm{Q}$ e $e_{2}(t)$ o vetor normal unitário perpendicular a $e_{1}(t)$ que aponta na direção do foco $\mathrm{F}$ de $\mathrm{Q}$. Denotamos por $T_{X(t)} Q$ a tangente a $\mathrm{Q}$ em $X(t)$. Ilustramos a seguir a quádrica $\mathrm{Q}$ após ela ter rolado sobre um eixo, sem deslizar, a uma certa distância em relação à sua posição inicial.

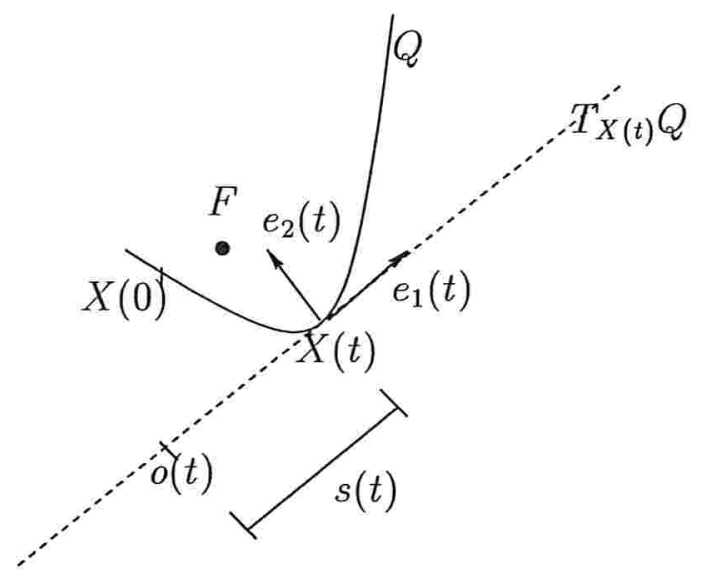

Figura 4.2: Representação de um sistema de coordenadas para Q no instante t

Geometricamente, sabemos que a origem $o(t)=X(0)=X\left(t_{0}\right)$ pode ser obtida retornando-se em $T_{X(t)} Q$ o comprimento de arco s(t). Ou seja,

$$
o(t)=X(t)-s(t) \cdot e_{1}(t) \in T_{X(t)} Q .
$$

Logo, para cada $t \in J$, tomamos $\left\{o(t) ; e_{1}(t), e_{2}(t)\right\}$ o referencial móvel definido sobre Q. Como estamos interessados em rolar Q sobre o eixo $O z$, então podemos representar o 
foco $\mathrm{F}$ de $\mathrm{Q}$ neste sistema como

$$
F=o(t)+z(t) \cdot e_{1}(t)+x(t) \cdot e_{2}(t)
$$

Com isto, $t \mapsto \gamma(t)=(x(t), 0, z(t)), t \in J$, é exatamente a trajetória de $\mathrm{F}$ quando Q rola sobre o eixo $O z$. Substituindo (4.41) em (4.42) obtemos:

$$
F=X(t)-s(t) e_{1}(t)+z(t) e_{1}(t)+x(t) e_{2}(t)
$$

Multiplicando $\mathrm{F}$ escalarmente por $e_{1}(t)$ e por $e_{2}(t)$ obtemos, respectivamente:

$$
\begin{aligned}
& F \cdot e_{1}(t)=X(t) \cdot e_{1}(t)-s(t)+z(t) \\
& F \cdot e_{2}(t)=X(t) \cdot e_{2}(t)+x(t)
\end{aligned}
$$

ou seja,

$$
\begin{gathered}
x(t)=(F-X(t)) \cdot e_{2}(t) \\
z(t)=s(t)+(F-X(t)) \cdot e_{1}(t),
\end{gathered}
$$

representam as coordenadas da trajetória de $\mathrm{F}$ dadas em função do referencial móvel.

\subsection{Superfícies de Delaunay}

Nesta seção examinamos cada uma das quádricas Q e obtemos especificamente, em cada caso, a expressão da trajetória de um de seus focos, quando Q rola, sem deslizar, sobre o eixo $O z$.

Para isto, provamos o resultado seguinte:

Teorema 4.4.1. As curvas $\gamma$ dos casos (3),(4) e (5) do Teorema 4.2.2 são geradas pela trajetória do foco de uma quádrica $Q$ não-degenerada que rola, sem deslizar, sobre o eixo Oz. Além disto,

(i) se $Q$ é a parábola $2 p x=z^{2}(p>0)$ então aparece como trajetória do foco uma catenária (caso 3) com $\rho_{1}=p / 2$,

(ii) se $Q$ é a elipse $\frac{(x-a)^{2}}{a^{2}}+\frac{z^{2}}{b^{2}}=1,0<b \leq a, e:=\sqrt{a^{2}-b^{2}}$ a semidistância focal, $k:=\frac{e}{a}$ a excentricidade de $Q$, então a trajetória 
$\gamma(t)=(x(t), 0, z(t))$ do foco de $Q$ é dada por:

$$
\begin{aligned}
& x(t)=b \cdot\left(\frac{1-k \cos t}{1+k \cos t}\right)^{1 / 2}, \\
& z(t)=\frac{b^{2}}{a} \int_{0}^{t} \frac{d \tau}{(1-k \cos \tau)^{1 / 2}(1+k \cos \tau)^{3 / 2}}, \quad \text { para } t \in \mathbb{R},
\end{aligned}
$$

que é uma solução do caso (4), onde:

$\rho_{1}=a-e ; \rho_{2}=a+e, H=\frac{1}{2 a} ; c=\frac{b^{2}}{2 a}$.

(iii) se $Q$ é a hipérbole $\frac{x^{2}}{a^{2}}-\frac{z^{2}}{b^{2}}=1, a, b>0, e:=\sqrt{a^{2}+b^{2}}$ a semidistância focal, $k:=\frac{e}{a}$ a excentricidade de $Q$, então a trajetória

$\gamma(t)=(x(t), 0, z(t))$ do foco de $Q$ é dada por:

$$
\begin{aligned}
x(t) & =b \cdot\left(\frac{k-\cos t}{k+\cos t}\right)^{1 / 2}, \\
z(t) & =\frac{b^{2}}{a} \cdot \int_{0}^{t} \frac{\cos \tau}{(k-\cos \tau)^{1 / 2}(k+\cos \tau)^{3 / 2}} d \tau, \quad \text { para } t \in \mathbb{R} ;
\end{aligned}
$$

que é uma solução do caso (5) com:

$\rho_{1}=e-a ; \rho_{2}=e+a \quad, H=-\frac{1}{2 a} ; c=\frac{b^{2}}{2 a}$.

Prova: Considerando os resultados obtidos na seção 4.3 e simplificando $\gamma(t)=(x(t), 0, z(t))$ para $\gamma(t)=(x(t), z(t))$, temos que:

(i) Se Q é a parábola $2 p x=z^{2}(p>0)$, então o foco $\mathrm{F}$ de $\mathrm{Q}$ é dado por $F=\left(\frac{p}{2}, 0\right)$.

Parametrizamos Q por: $X(t)=\left(\frac{t^{2}}{2 p}, t\right), t \in \mathbb{R}$.

Logo, $\dot{X}(t)=\left(\frac{t}{p}, 1\right)$ e $\|\dot{X}(t)\|=\left(\frac{t^{2}}{p^{2}}+1\right)^{1 / 2}$.

Observamos que, devido à expressão de $\|\dot{X}(t)\|$, temos $\|\dot{X}(t)\| \neq 0, \forall t \in \mathbb{R}$.

Então, temos

$e_{1}(t)=\frac{1}{\left(\frac{t^{2}}{p^{2}}+1\right)^{1 / 2}}\left(\frac{t}{p}, 1\right), \quad e_{2}(t)=\frac{1}{\left(\frac{t^{2}}{p^{2}}+1\right)^{1 / 2}}\left(1,-\frac{t}{p}\right)$ e $\quad s(t)=\int_{0}^{t}\left(1+\frac{\tau^{2}}{p^{2}}\right)^{1 / 2} d \tau$.

Fazendo a substituição $\tau=p$.senh $u$, resulta que:

$s(t)=\frac{t}{2}\|\dot{X}(t)\|+\frac{p}{2} \operatorname{arcsinh}\left(\frac{t}{p}\right)$. Por (4.44) e (4.45) obtemos que

$$
x(t)=\frac{p}{2}\|\dot{X}(t)\|=\frac{p}{2}\left(1+\frac{t^{2}}{p^{2}}\right)^{1 / 2}
$$


$\mathrm{e}$

$$
z(t)=\frac{t}{2}\|\dot{X}(t)\|+\frac{p}{2} \operatorname{arcsinh}\left(\frac{t}{p}\right)-\frac{t}{2}\|\dot{X}(t)\|=\frac{p}{2} \operatorname{arcsinh}\left(\frac{t}{p}\right) .
$$

Logo, $\frac{2 z(t)}{p}=\operatorname{arcsinh}\left(\frac{t}{p}\right)$ e consequentemente, temos:

$$
\cosh \left(\frac{2 z(t)}{p}\right)=\cosh \left(\operatorname{arcsinh}\left(\frac{t}{p}\right)\right)=\left(1+\frac{t^{2}}{p^{2}}\right)^{1 / 2}=\frac{2 x(t)}{p} .
$$

Portanto, obtemos que a trajetória do foco $\mathrm{F}$ de $\mathrm{Q}$, quando $\mathrm{Q}$ rola sobre o eixo $O z$, é dada por:

$$
x(t)=\frac{p}{2} \cosh \left(\frac{z(t)}{p / 2}\right), t \in \mathbb{R},
$$

que é a equação de uma catenária. Portanto, temos que este caso corresponde ao caso (3) do Teorema $4.2 .2, \operatorname{com} \rho_{1}=p / 2$. Ou seja, a curva $\gamma$ deste caso é gerada pela trajetória do foco de uma parábola. Denominamos a superfície de revolução obtida de catenóide.

(ii) Se Q é a elipse $\frac{(x-a)^{2}}{a^{2}}+\frac{z^{2}}{b^{2}}=1,0<b \leq a, e:=\sqrt{a^{2}-b^{2}}, k:=\frac{e}{a}$,

então escolhemos para ser o foco $\mathrm{F}$ aquele que está mais próximo da origem do sistema de coordenadas fixado do plano $O x z$, isto é, $F=(a-e, 0)$.

Parametrizamos Q por: $X(t)=(a-a \cos t, b \sin t), t \in \mathbb{R}$.

Logo, $\dot{X}(t)=(a \sin t, b \cos t) \mathrm{e}$

$\|\dot{X}(t)\|=a \sqrt{1-k^{2} \cos ^{2} t}$, visto que $k^{2}=\frac{e^{2}}{a^{2}}=\frac{a^{2}-b^{2}}{a^{2}}=1-\frac{b^{2}}{a^{2}}$, isto é, $\frac{b^{2}}{a^{2}}=1-k^{2}$.

Observamos que, como $0<b \leq a$, então $0 \leq k<1$. Logo, $1-k \cos t \geq 1-k>0$ e $1+k \cos t \geq 1-k>0$.

Assim, $1-k^{2} \cos ^{2} t=(1-k \cos t)(1+k \cos t)>0$ e, portanto, $\|\dot{X}(t)\| \neq 0, \forall t \in \mathbb{R}$, visto que $a>0$.

Tomamos então

$e_{1}(t)=\frac{1}{a \sqrt{1-k^{2} \cos ^{2} t}}(a \sin t, b \cos t)$ e $e_{2}(t)=\frac{1}{a \sqrt{1-k^{2} \cos ^{2} t}}(b \cos t,-a \sin t)$. Logo,

$$
s(t)=\int_{0}^{t} a \sqrt{1-k^{2} \cos ^{2} \tau} d \tau
$$


Por (4.44) e (4.45) obtemos que

$$
\begin{aligned}
x(t) & =\frac{a b-e b \cos t}{a(1-k \cos t)^{1 / 2}(1+k \cos t)^{1 / 2}}=\frac{a b(1-k \cos t)}{a(1-k \cos t)^{1 / 2}(1+k \cos t)^{1 / 2}}=b\left(\frac{1-k \cos t}{1+k \cos t}\right)^{1 / 2} \\
e & \\
z(t) & =s(t)+\frac{e^{2} \sin t \cos t-e a \sin t}{a(1-k \cos t)^{1 / 2}(1+k \cos t)^{1 / 2}}=s(t)+\frac{-e a \sin t(1-k \cos t)}{a(1-k \cos t)^{1 / 2}(1+k \cos t)^{1 / 2}}= \\
& =\int_{0}^{t} a \cdot \sqrt{1-k^{2} \cos ^{2} \tau} d \tau-e \sin t\left(\frac{1-k \cos t}{1+k \cos t}\right)^{1 / 2}=\int_{0}^{t} a \cdot \sqrt{1-k^{2} \cos ^{2} \tau} d \tau-\frac{e}{b} x(t) \sin t
\end{aligned}
$$

de onde resulta que $z(0)=0$.

Agora, vamos tentar reescrever $z(t)$ na forma em que aparece em (ii) neste Teorema. Calculando-se obtemos:

$$
\begin{aligned}
& \dot{x}(t)=\frac{e}{a} b \frac{\sin t}{(1-k \cos t)^{1 / 2}(1+k \cos t)^{3 / 2}} \\
& e \\
& \dot{z}(t)=\frac{a^{2}\left(1-k^{2} \cos ^{2} t\right)(1+k \cos t)-e a\left(1-k^{2} \cos ^{2} t\right) \cos t-e^{2} \sin ^{2} t}{a(1-k \cos t)^{1 / 2}(1+k \cos t)^{3 / 2}} .
\end{aligned}
$$

Visto que $a k=e$ e $b^{2}=a^{2}-e^{2}$, temos então

$$
\dot{z}(t)=\frac{b^{2}}{a(1-k \cos t)^{1 / 2}(1+k \cos t)^{3 / 2}} .
$$

Integrando esta expressão obtemos

$$
z(t)=\int_{0}^{t} \frac{b^{2}}{a(1-k \cos \tau)^{1 / 2}(1+k \cos \tau)^{3 / 2}} d \tau+\alpha
$$

onde $\alpha$ é uma constante de integração. Como tínhamos que $z(0)=0$, obtemos finalmente

$$
z(t)=\frac{b^{2}}{a} \int_{0}^{t} \frac{d \tau}{(1-k \cos \tau)^{1 / 2}(1+k \cos \tau)^{3 / 2}}, t \in \mathbb{R}
$$

Com isto, determinamos a expressão em coordenadas da trajetória $\gamma(t)=(x(t), z(t))$ do foco de Q, conforme aparece no caso (ii) deste teorema. Falta mostrar que este caso corresponde ao caso (4) do Teorema 4.2.2.

Começamos observando que devido à expressão de $\dot{z}(t)$ obtida há pouco, tínhamos $\dot{z}(t)>0, \forall t$. Isto é, $\dot{z}(t) \neq 0, \forall t \in J$. 
Lembramos que no caso $H=$ constante, mostramos que vale para uma curva $\gamma(t)=$ $(x(t), 0, z(t))$ a expressão (4.5) e portanto, ela deve ser satisfeita pela curva $\gamma$ acima obtida. Calculamos:

$$
\begin{aligned}
\sqrt{\dot{x}^{2}(t)+\dot{z}^{2}(t)} & =\left(\frac{e^{2} b^{2} \sin ^{2} t+b^{4}}{a^{2}(1-k \cos t)(1+k \cos t)^{3}}\right)^{1 / 2}=\frac{b}{1+k \cos t}\left(\frac{b^{2}+e^{2}\left(1-\cos ^{2} t\right)}{a^{2}\left(1-k^{2} \cos ^{2} t\right)}\right)^{1 / 2} \\
& =\frac{b}{1+k \cos t}\left(\frac{a^{2}-e^{2} \cos ^{2} t}{\left.a^{2}-e^{2} \cos ^{2} t\right)}\right)^{1 / 2}=\frac{b}{1+k \cos t},
\end{aligned}
$$

visto que $b>0,1+k \cos t>0, b^{2}+e^{2}=a^{2}, a^{2} k^{2}=e^{2}$.

Consequentemente,

$$
\begin{aligned}
\frac{\dot{z}(t)}{\sqrt{\dot{x}^{2}(t)+\dot{z}^{2}(t)}} & =\frac{b}{a(1-k \cos t)^{1 / 2}(1+k \cos t)^{1 / 2}}=\frac{b}{2 a}\left(\frac{1-k \cos t}{1+k \cos t}\right)^{1 / 2}+\frac{b}{2 a}\left(\frac{1+k \cos t}{1-k \cos t}\right)^{1 / 2}= \\
& =\frac{1}{2 a} x(t)+\frac{b^{2}}{2 a} \frac{1}{x(t)} .
\end{aligned}
$$

Esta é exatamente a expressão (4.5) com $H=\frac{1}{2 a}$ e $c=\frac{b^{2}}{2 a}$ e portanto, estamos no caso $H, c \neq 0$.

A seguir, mostramos que a trajetória $\gamma$ de $\mathrm{F}$ varia entre $\rho_{1}=a-e$ e $\rho_{2}=a+e$. De fato, temos que $\rho_{1}=\inf _{t \in J} x(t), \quad \rho_{2}=\sup _{t \in J} x(t)$, onde $x(t)=b\left(\frac{1-k \cos t}{1+k \cos t}\right)^{1 / 2}$. Do Cálculo, sabemos que os máximos e mínimos relativos de $x(t)$, no aberto $\mathrm{J}$, devem satisfazer $\dot{x}(t)=0$. Mas, pela expressão de $\dot{x}(t), \dot{x}(t)=0 \Longleftrightarrow \sin t=0 \Longleftrightarrow \cos ^{2} t=1$.

Para cos $t=1$ obtemos: $x(t)=b\left(\frac{1-k}{1+k}\right)^{1 / 2}=b\left(\frac{a-e}{a+e}\right)^{1 / 2}=b\left(\frac{(a-e)^{2}}{a^{2}-e^{2}}\right)^{1 / 2}=a-e$, pois $a^{2}-e^{2}=b^{2}, b>0$ e $a-e>0$.

Para $\cos t=-1$ obtemos: $x(t)=b\left(\frac{1+k}{1-k}\right)^{1 / 2}=b\left(\frac{a+e}{a-e}\right)^{1 / 2}=b\left(\frac{(a+e)^{2}}{a^{2}-e^{2}}\right)^{1 / 2}=a+e$.

Além disto, se calcularmos a expressão de $\ddot{x}(t)$ podemos verificar facilmente que $\ddot{x}(2 n \pi)>0, n \in\{0,1,2, \ldots\}$, e portanto, neste caso obtemos um mínimo loca.l Da mesma forma, $\ddot{x}(\pi+2 n \pi)<0, n \in\{0,1,2, \ldots\}$, e portanto neste caso obtemos um máximo local.

Pela expressão de $x(t)$ vemos ainda que ela é uma função periódica em $\mathrm{J}$, de onde concluímos que: $\rho_{1}=a-e$ e $\rho_{2}=a+e$.

Visto que $\operatorname{sgn} \dot{z}(t)=+1$, pois $\dot{z}(t)>0, \forall t \in J$, concluímos:

$$
H=\frac{1}{2 a}=\frac{\operatorname{sgn} \dot{z}(t)}{(a-e)+(a+e)}=\frac{1}{\rho_{1}+\rho_{2}} \operatorname{sgn} \dot{z}(t)
$$


e

$$
c=\frac{b^{2}}{2 a}=\frac{a^{2}-e^{2}}{(a-e)+(a+e)} \operatorname{sgn} \dot{z}(t)=\frac{\rho_{1} \cdot \rho_{2}}{\rho_{1}+\rho_{2}} \operatorname{sgn} \dot{z}(t)
$$

Portanto, vemos que este caso corresponde ao caso (4), do Teorema 4.2.2. Ou seja, a curva $\gamma$, neste caso, é gerada pela trajetória do foco de uma elipse. Denominamos $\gamma$ de ondulária e a superfície de revolução chamamos de ondulóide.

Observamos que quando $k=0$, isto é, $a=b$, o ondulóide é exatamente um cilindro. De fato, neste caso a equação da elipse se torna $(x-a)^{2}+z^{2}=a^{2}$ e seu foco é então dado por $F=(a, 0)$, cuja trajetória descreve uma reta paralela ao eixo $O z$ e portanto, a respectiva superfície de revolução é um cilindro. Temos ainda que $\rho_{1}=\rho_{2}$, pois $k=\frac{e}{a}=0$ e portanto, este caso corresponde ao subcaso (i) do caso (4) do Teorema 4.2.2. Além disto, para valores de $\mathrm{k}$ cada vez mais próximos de 1 , temos que $\rho_{1} \rightarrow 0 \mathrm{e}$, consequentemente, $H \rightarrow \frac{1}{\rho_{2}} \operatorname{sgn} \dot{z}$ e $c \rightarrow 0$ e portanto, obteríamos o caso (2) do Teorema 4.2.2. Ou seja, quando k se aproxima de 1 , o ondulóide se aproxima de uma esfera.

(iii) Se Q é a hipérbole $\frac{x^{2}}{a^{2}}-\frac{z^{2}}{b^{2}}=1, a, b>0, e:=\sqrt{a^{2}+b^{2}}, k:=\frac{e}{a}$, escolhemos $F=(e, 0)$ para ser o foco de $\mathrm{Q}$.

Parametrizamos $\mathrm{Q}$ por $X(t)=\left(\frac{a}{\cos t}, b \operatorname{tg} t\right)$, para $t \in \mathbb{R} \operatorname{com} \cos t \neq 0$.

Logo, $\dot{X}(t)=\frac{1}{\cos ^{2} t}(a \sin t, b)$, para $t \in \mathbb{R} \operatorname{com} \cos t \neq 0$.

$\mathrm{e}$

$$
\begin{aligned}
\|\dot{X}(t)\| & =\frac{1}{\cos ^{2} t}\left(a^{2} \sin ^{2} t+b^{2}\right)^{1 / 2}=\frac{a}{\cos ^{2} t}\left(\sin ^{2} t+\frac{b^{2}}{a^{2}}\right)^{1 / 2}= \\
& =\frac{a}{\cos ^{2} t}\left(1-\cos ^{2} t+\frac{b^{2}}{a^{2}}\right)^{1 / 2}=\frac{a}{\cos ^{2} t}\left(k^{2}-\cos ^{2} t\right)^{1 / 2},
\end{aligned}
$$

para $t \in \mathbb{R} \operatorname{com} \cos t \neq 0$, visto que $k^{2}=\frac{e^{2}}{a^{2}}=1+\frac{b^{2}}{a^{2}}$.

Como $a, b>0$ e $k=\frac{\sqrt{a^{2}+b^{2}}}{a}$ então $k>1$. Como $-1 \leq \cos t \leq 1$, então $k-\cos t \geq$ $k-1>0$ e $k+\cos t \geq k-1>0$. Consequentemente, $k^{2}-\cos ^{2} t=(k-\cos t)(k+\cos t)>0$ e portanto, $\|\dot{X}(t)\| \neq 0, \forall t \in \mathbb{R} \operatorname{com} \cos t \neq 0$.

Tomamos $e_{1}(t)=\frac{1}{a\left(k^{2}-\cos ^{2} t\right)^{1 / 2}}(a \sin t, b)$ e $e_{2}(t)=\frac{1}{a\left(k^{2}-\cos ^{2} t\right)^{1 / 2}}(b,-a \sin t)$.

Agora, como obtemos $\|\dot{X}(t)\|$ somente para $t \in \mathbb{R}$ com cos $t \neq 0$, podemos definir o comprimento de arco no intervalo em que $\cos t \neq 0$, como por exemplo, em intervalos consecutivos: 


$$
\left.s(t)=a \int_{0}^{t} \frac{\left(k^{2}-\cos ^{2} \tau\right)^{1 / 2}}{\cos ^{2} \tau} d \tau, \text { para } t \in I:=\right]-\pi / 2, \pi / 2[,
$$

ou

$$
\left.s_{*}(t)=a \int_{\pi}^{t} \frac{\left(k^{2}-\cos ^{2} \tau\right)^{1 / 2}}{\cos ^{2} \tau} d \tau, \quad \text { para } \quad t \in I_{*}:=\right] \pi / 2,3 \pi / 2[,
$$

e assim por diante.

Por (4.44) e (4.45) obtemos que

$x(t)=\frac{b}{(k-\cos t)^{1 / 2}(k+\cos t)^{1 / 2}}\left(k-\frac{1}{\cos t}+\frac{\sin ^{2} t}{\cos t}\right)=b\left(\frac{k-\cos t}{k+\cos t}\right)^{1 / 2}$

$\mathrm{e}$

$z(t)=s(t)-\frac{e \operatorname{atg} t}{a\left(k^{2}-\cos ^{2} t\right)^{1 / 2}}(k-\cos t)=s(t)-e \operatorname{tg} t \frac{x(t)}{b}$, para $t \in I$,

de onde segue que $z(0)=0 \mathrm{e}$, respectivamente,

$z_{*}(t)=s_{*}(t)-e \operatorname{tg} t \frac{x(t)}{b}$, para $t \in I_{*}$,

e assim por diante.

A seguir, vamos analisar o comportamento das funções $z(t)$ e $z_{*}(t)$, que estão definidas nos intervalos consecutivos $I:=]-\pi / 2, \pi / 2\left[\right.$ e $\left.\quad I_{*}:=\right] \pi / 2,3 \pi / 2[$, a fim de obtermos uma expressão geral para $z(t)$.

Começamos calculando:

$\dot{x}(t)=\frac{b \sin t}{2(k-\cos t)^{1 / 2}(k+\cos t)^{3 / 2}}(k+\cos t+k-\cos t)=\frac{b e}{a} \frac{\sin t}{(k-\cos t)^{1 / 2}(k+\cos t)^{3 / 2}}$.

Logo, para $t \in I$, temos

$$
\begin{aligned}
\dot{z}(t) & =\dot{s}(t)-e \frac{1}{\cos ^{2} t} \frac{x(t)}{b}-e \operatorname{tg} t \frac{\dot{x}(t)}{b}= \\
& =\frac{a(k-\cos t)(k+\cos t)[a(k+\cos t)-e]-e^{2} \sin ^{2} t \cos t}{a \cos ^{2} t(k-\cos t)^{1 / 2}(k+\cos t)^{3 / 2}}= \\
& =\frac{b^{2} \cos ^{2}}{a(k-\cos t)^{1 / 2}(k+\cos t)^{3 / 2}}
\end{aligned}
$$

e, para $t \in I_{*}$, temos de forma análoga

$\dot{z}_{*}(t)=\dot{s}_{*}(t)-e \frac{1}{\cos ^{2} t} \frac{x(t)}{b}-e \operatorname{tg} t \frac{\dot{x}(t)}{b}=\frac{b^{2} \cos t}{a(k-\cos t)^{1 / 2}(k+\cos t)^{3 / 2}}$.

Ou seja, $\dot{z}(t)$ e $\dot{z}_{*}(t)$ têm as mesmas expressões, apesar de estarem calculados em diferentes t. Além disto, são limitadas por $\frac{b^{2}}{a(k-1)^{2}}$. Isto segue do fato que $k-\cos t>$ $k-1$ e $k+\cos t>k-1$.

Do Cálculo Diferencial segue que $z(t)$ e $z_{*}(t)$ são funções Lipschitzianas e portan- 
to, uniformemente contínuas. Por um resultado de Análise ( ver [18] ), segue que existem $z(\pi / 2)=\lim _{\substack{t \rightarrow \pi / 2 \\ t \in I}} z(t)$ e $z_{*}(\pi / 2)=\lim _{\substack{t \rightarrow \pi / 2 \\ t \in I_{*}}} z_{*}(t)$.

Pelas expressões de $\dot{z}(t)$ e $\dot{z}_{*}(t)$, temos $\dot{z}(\pi / 2)=\dot{z}_{*}(\pi / 2)=0$, ou seja, $z(t)$ e $z_{*}(t)$ têm a mesma tangente em $\pi / 2$. Desta forma, podemos prolongar $z(t)$ e $z_{*}(t)$ para $t=\pi / 2$.

No entanto, tínhamos que as respectivas funções $z(t)$ ( que chamamos de $z(t), z_{*}(t)$, $z_{* *}(t)$ e assim por diante ) foram definidas em intervalos consecutivos. Além disto, podemos repetir a análise feita para $z(t)$ e $z_{*}(t)$ também para os outros $t$ com $\cos t=0$, visto que $\dot{z}(t), \dot{z}_{*}(t), \dot{z}_{* *}(t)$, e assim por diante, têm as mesmas expressões. Concluímos então que $z(t)$ pode ser prolongada para $t \in \mathbb{R}$. Ou seja,

$$
z(t)=\frac{b^{2}}{a} \int_{0}^{t} \frac{\cos \tau}{(k-\cos \tau)^{1 / 2}(k+\cos \tau)^{3 / 2}} d \tau+\alpha, \text { onde } \alpha \text { é uma constante de inte- }
$$
gração, $t \in \mathbb{R}$.

Como tínhamos que $z(0)=0$, então $z(t)=\frac{b^{2}}{a} \int_{0}^{t} \frac{\cos \tau}{(k-\cos \tau)^{1 / 2}(k+\cos \tau)^{3 / 2}} d \tau$, $t \in \mathbb{R}$. Com isto, obtemos a trajetória $\gamma(t)=(x(t), z(t))$ do foco $\mathrm{F}$ de $\mathrm{Q}$, conforme apresentado no item (iii) deste teorema.

Falta mostrar que este caso corresponde ao caso (5) do Teorema 4.2.2. Primeiramente observamos que neste caso existe, no mínimo, um $t_{0}$ tal que $\dot{z}\left(t_{0}\right)=0$; por exemplo, $t_{0}=\pi / 2$. Procedendo como no item (ii), calculamos:

$$
\sqrt{\dot{x}^{2}(t)+\dot{z}^{2}(t)}=\frac{1}{a(k-\cos t)^{1 / 2}(k+\cos t)^{3 / 2}}\left(e^{2} b^{2} \sin ^{2} t+b^{4} \cos ^{2} t\right)^{1 / 2}=\frac{b}{k+\cos t}
$$

$\mathrm{e}$

$$
\begin{aligned}
\frac{\dot{z}(t)}{\sqrt{\dot{x}^{2}(t)+\dot{z}^{2}(t)}} & =\frac{b \cos t}{a(k-\cos t)^{1 / 2}(k+\cos t)^{3 / 2}}=-\frac{b}{2 a}\left(\frac{k-\cos t}{k+\cos t}\right)^{1 / 2}+\frac{b}{2 a}\left(\frac{k+\cos t}{k-\cos t}\right)^{1 / 2}= \\
& =-\frac{1}{2 a} x(t)+\frac{b^{2}}{2 a} \frac{1}{x(t)} .
\end{aligned}
$$

Mas, esta é exatamente a equação diferencial (4.5) com $H=-\frac{1}{2 a}$ e $c=\frac{b^{2}}{2 a}$.

Procedendo novamente de forma análoga ao item (ii) para calcular $\rho_{1}=\inf _{t \in J} x(t)$, $\rho_{2}=\sup _{t \in J} x(t)$, concluímos que $\rho_{1}=e-a$ e $\rho_{2}=e+a$. Pela expressão de $\dot{z}(t)$ temos que $\dot{z}(t) \geq 0, \forall t \in J$. Além disto, $\dot{z}_{1}(t)>0$, onde $t_{1} \in J$ é tal que $x\left(t_{1}\right)=\rho_{1}$, pois, caso contrário, teríamos por (4.5) 
$0=\dot{z}\left(t_{1}\right)=H \rho_{1}+\frac{c}{\rho_{1}}$, isto é, $\rho_{1}^{2}=-\frac{c}{H} \Longrightarrow \rho_{1}=0$, o que é absurdo, pois no caso em que $H, c \neq 0$ temos $\rho_{1}>0$. Logo, sgn $\dot{z}\left(t_{1}\right)=+1$,

$H=-\frac{1}{2 a}=-\frac{1}{(e+a)-(e-a)} \operatorname{sgn} \dot{z}\left(t_{1}\right)=-\frac{1}{\rho_{2}-\rho_{1}} \operatorname{sgn} \dot{z}\left(t_{1}\right)$

$c=\frac{b^{2}}{2 a}=\frac{e^{2}-a^{2}}{(e+a)-(e-a)} \operatorname{sgn} \dot{z}\left(t_{1}\right)=\frac{\rho_{1} \rho_{2}}{\rho_{2}-\rho_{1}} \operatorname{sgn} \dot{z}\left(t_{1}\right)$.

Portanto, vemos que este caso corresponde ao caso (5) do Teorema 4.2.2. Ou seja, a curva $\gamma$, neste caso, é gerada pela trajetória do foco de uma hipérbole. Denominamos $\gamma$ de nodária e a superfície de revolução chamamos de nodóide.

Observação 4.4.2. Seria natural parametrizar a hipérbole considerada no item (iii) do Teorema 4.4.1 por

$$
X(t)=(a \cosh t, b \sinh t), \quad t \in \mathbb{R} .
$$

Justificamos agora o motivo desta parametrização não ter sido tomada.

Primeiramente, notamos que esta parametrização cobre apenas o ramo de hipérbole que tem $x>0$. Procedendo exatamente como em (iii), obteríamos:

$$
\begin{aligned}
& x(t)=b\left(\frac{k \cosh t-1}{k \cosh t+1}\right)^{1 / 2}, z(t)=\frac{b^{2}}{a} \int_{0}^{t} \frac{b^{2}}{(k \cosh \tau-1)^{1 / 2}(k \cosh \tau+1)^{3 / 2}} e \\
& \dot{z}(t)=\frac{}{a(k \cosh t-1)^{1 / 2}(k \cosh t+1)^{3 / 2}} .
\end{aligned}
$$

Com isto, temos $\dot{z}(t) \neq 0, \forall t \in \mathbb{R}$, pois $\dot{z}(t)>0$. Além disto, temos $\frac{\dot{z}(t)}{\sqrt{\dot{x}^{2}(t)+\dot{z}^{2}(t)}}=-\frac{1}{2 a} x(t)+\frac{b^{2}}{2 a} \frac{1}{x(t)}$, que é exatamente a equação diferencial (4.5) com $H=-\frac{1}{2 a}$ e $c=\frac{b^{2}}{2 a}$, ou seja, estamos no caso $H, c \neq 0$. Calculando $\rho_{1}=\inf _{t \in J} x(t)$, $\rho_{2}=\sup _{t \in J} x(t)$, onde $x(t)$ foi dada acima, temos $\rho_{1}=e-a$ e $\rho_{2}=b$, isto é, $\rho_{1}>0 e$ $\rho_{2}<\infty$. No entanto, não é possível mostrar, com as expressões obtidas, que este caso corresponde a um dos casos do Teorema 4.2.2 e, como sabemos, todas as possibilidades para o valor de $H$ e c estão dadas neste teorema. Com isto, vemos que a parametrização tomada no início desta observação não é a melhor para caracterizar a curva denominada nodária.

Observação 4.4.3. Conforme observamos na introdução do capítulo 4, segue da classificação feita nos Teoremas 4.2.2 e 4.4.1 que as soluçôes do problema (P) são determinadas 
por aqueles pedaços das superfícies de Delaunay limitados entre os planos paralelos $P_{1} e$ $P_{2}$ e com bordo contido nestes planos. Portanto, temos que as possiveis soluções de $(P)$ são o cilindro, o catenóide, o nodóide, o ondulóide, a esfera (respectivamente a semiesfera se colocarmos como hipótese o bordo estar contido somente em um dos planos $\left.P_{i}\right)$ e o plano, tomando, para todas estas superfícies, o bordo contido nos planos $P_{1}$ e/ou $P_{2}$.

Conclusão Devido às condições do problema ( $\mathrm{P}$ ) interessa-nos somente a esfera ( ou a semiesfera), o cilindro e o ondulóide, incluindo-se os seus bordos. As demais superfícies serão descartadas na análise posterior, visto que o plano não delimita um um sólido, o catenóide nunca encontra os planos $P_{1}$ e $P_{2}$ perpendicularmente e o nodóide tem auto-intersecções. 
CAPÍtulo 5

\section{Estabilidade das soluções do problema $(\mathbf{P})$}

De acordo com a conclusão do capítulo 4, temos que uma esfera localizada entre os planos paralelos $P_{1}$ e $P_{2}$ é uma possível solução do problema variacional (P). No entanto, por meio de alguns cálculos simples mostramos que a semiesfera, que encerra o mesmo volume $\mathrm{V}$ da esfera, tem área superficial menor. Como estamos interessados em determinar o mínimo do funcional área, então tomamos como uma possível solução de (P) uma semiesfera, ao invés da esfera. Também queremos que a superfície encontre os planos $P_{1}$ e $P_{2}$ perpendicularmente ao longo de seu bordo e, por esta razão, apoiamos tal semiesfera sobre um dos planos $P_{1}$ ou $P_{2}$.

Pelo mesmo motivo acima, no caso do ondulóide também só estaremos interessados no pedaço desta superfície que está entre os planos $P_{1}$ e $P_{2}$ e os encontra perpendicularmente. Como consideraremos neste capítulo a curva geratriz do ondulóide dada por $\gamma(t)=(x(t), t)$, temos que o ondulóide encontra os planos perpendicularmente nos pontos de $\gamma(t)$ tais que $\dot{x}(t)=0$. De fato, se $\dot{x}(t)=0$, então $\dot{\gamma}(t)=(\dot{x}(t), 1)=(0,1)$, que é um vetor no plano $0 x z$ paralelo ao eixo $0 z$ ( ver Figura 5.1 ).

O principal resultado deste capítulo é a demonstração da instabilidade do ondulóide, que é um resultado surpreendente, pois contradiz o experimento das películas de sabão. Devido às condições do problema $(\mathrm{P})$, estamos interessados em perturbações que mantêm o volume fixado. Tomamos, desta forma, uma perturbação de um cilindro, apoiado sob duas lâminas paralelas $\mathfrak{L}_{1}$ e $\mathfrak{L}_{2}$, que mantém fixado seu volume e aumentamos lentamente 


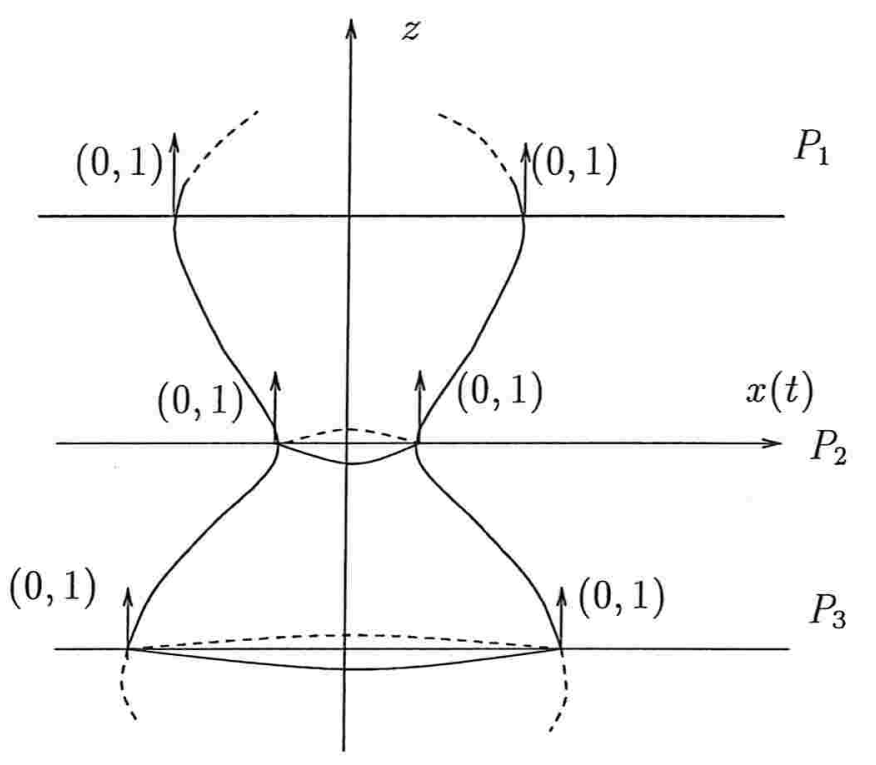

Figura 5.1: Posições dos vetores $\dot{\gamma}(t)=(0,1)$ em relação ao ondulóide

a distância entre as lâminas; então é possível constatar, neste experimento, que o cilindro é transformado continuamente em uma superfície que tem a forma de um ondulóide e, na verdade, um semiperíodo dele. Suponhamos que o ondulóide tenha aparecido com a alça menor apoiada sob a lâmina $\mathfrak{L}_{1}$ e a maior sob a lâmina $\mathfrak{L}_{2}$. Constata-se ainda, neste experimento, que é possível obter uma perturbação de tal ondulóide, que mantém seu volume fixado, e que o modifica lentamente de modo que a alça menor passa a estar apoiada sobre $\mathfrak{L}_{2}$ e a maior sobre $\mathfrak{L}_{1}$, o que justifica a estabilidade física do ondulóide.

Uma possível explicação para a diferença acima apontada é que a gravidade não é considerada no modelo matemático. Para um tratamento do problema variacional (P) do ponto de vista físico, damos como referência o trabalho de Voguel [22], onde é considerada uma gota líquida prensada entre duas lâminas paralelas.

A fim de provar a instabilidade do ondulóide, introduzimos na próxima seção os resultados e definições que nos conduzirão a tal prova. 


\subsection{A segunda variação}

Conforme comentamos no final do capítulo 4, as possíveis soluções de (P) são somente a semiesfera, o cilindro e o ondulóide ( com bordo contido nos planos $P_{1}$ e $P_{2}$ ). Então podemos parametrizar a curva $\gamma(t)$ como um gráfico na forma:

$$
\gamma(t)=(x(t), t), x(t)>0, \quad t \in[0, d], \quad d:=\operatorname{dist}\left(P_{1}, P_{2}\right) .
$$

Daqui em diante também estaremos considerando a curva em $\gamma(t)=(x(t), 0, t) \subset \mathbb{R}^{3}$ no plano $O x z$, isto é, $\gamma(t)=(x(t), t)$. Logo, a parametrização (4.3) da superfície rotacional S se torna

$$
\varphi(t, \phi)=(x(t) \cos \phi, x(t) \sin \phi, t), \quad \phi \in[0,2 \pi[.
$$

De forma análoga ao que observamos para o ondulóide na introdução deste capítulo, queremos que a superfície $\mathrm{S}$ encontre os planos $P_{1}$ e $P_{2}$ perpendicularmente ao longo de seu bordo $\partial S$, que por (5.1) é obtido para $t=0$ e $t=d$. Devido à expressão de $\gamma(t)=(x(t), t)$ no plano $O x z$, isto equivale a supor então que

$$
\dot{x}(0)=\dot{x}(d)=0 \text {. }
$$

Calculamos então os coeficientes $\mathrm{E}, \mathrm{F}, \mathrm{G}$ da $1^{\stackrel{a}{a}}$ forma fundamental e os coeficientes e, $\mathrm{f}, \mathrm{g}$ da $2^{\underline{a}}$ forma fundamental de S. Utilizando (5.1) temos que

$$
\begin{aligned}
& E=\dot{x}^{2}+1, \\
& F=0, \\
& G=x^{2},
\end{aligned}
$$$$
e=-\frac{\ddot{x}}{\sqrt{1+\dot{x}^{2}}},
$$$$
f=0 \text {, }
$$$$
g=\frac{x}{\sqrt{1+\dot{x}^{2}}} \text {. }
$$

Além disto, o campo normal unitário a S é dado por

$$
N(t, \phi)=\frac{1}{\sqrt{1+\dot{x}^{2}}}(-\cos \phi,-\sin \phi, \dot{x})
$$

e as curvaturas principais de $\mathrm{S}$ são

$$
k_{1}=\frac{e}{E}=-\frac{\ddot{x}}{\left(1+\dot{x}^{2}\right)^{3 / 2}} \quad \text { e } \quad k_{2}=\frac{g}{G}=\frac{1}{x \sqrt{1+\dot{x}^{2}}} .
$$

Consequentemente,

$$
2 H=k_{1}+k_{2}=-\frac{\ddot{x}}{\left(1+\dot{x}^{2}\right)^{3 / 2}}+\frac{1}{x\left(1+\dot{x}^{2}\right)^{1 / 2}} .
$$


Visto que a superfície S é obtida pela rotação da curva $\gamma(t)$, dada como gráfico da função $x=x(z)$, em torno do eixo $O z$, então a área de $\mathrm{S}$ é dada por

$$
A(S)=\int_{0}^{2 \pi} \int_{0}^{d} \sqrt{x^{2}\left(1+\dot{x}^{2}\right)} d t d \phi=2 \pi \int_{0}^{d} x \sqrt{1+\dot{x}^{2}} d t=A(x)
$$

e o volume encerrado pela superfície rotacional S é

$$
V(S)=\pi \int_{0}^{d} x^{2}(t) d t=V(x) .
$$

Determinamos agora uma expressão para $A^{\prime}(0)$ e $V^{\prime}(0)$, quando consideramos uma variação da forma $\left.x_{\epsilon}(t)=x(t)+\epsilon \psi(t), \epsilon \in\right]-\epsilon_{0}, \epsilon_{0}\left[\operatorname{com} \psi \in C^{2}([0, d])\right.$.

Temos por (5.7) que $A\left(x_{\epsilon}\right)=2 \pi \int_{0}^{d}(x+\epsilon \psi) \sqrt{1+(\dot{x}+\epsilon \dot{\psi})^{2}} d t$ e denotando por " " " a derivação em relação a $\epsilon$, segue que

$$
A^{\prime}(\epsilon):=\frac{d}{d \epsilon} A\left(x_{\epsilon}\right)=2 \pi \int_{0}^{d}\left\{\psi \sqrt{1+(\dot{x}+\epsilon \dot{\psi})^{2}} d t+(x+\epsilon \psi) \frac{(\dot{x}+\epsilon \dot{\psi}) \dot{\psi}}{\sqrt{1+(\dot{x}+\epsilon \dot{\psi})^{2}}}\right\} d t
$$

Fazendo $\epsilon=0$ na expressão acima, obtemos

$$
A^{\prime}(0)=2 \pi \int_{0}^{d} \psi \sqrt{1+\dot{x}^{2}} d t+2 \pi \int_{0}^{d} \frac{x \dot{x} \dot{\psi}}{\sqrt{1+\dot{x}^{2}}} d t
$$

Utilizando-se integração por partes e (5.2), obtemos

$$
\begin{aligned}
\int_{0}^{d} \frac{x \dot{x}}{\sqrt{1+\dot{x}^{2}}} \dot{\psi} d t & =\left.\frac{x \dot{x} \psi}{\sqrt{1+\dot{x}^{2}}}\right|_{0} ^{d}-\int_{0}^{d}\left\{\frac{\dot{x}^{2}+x \ddot{x}+\dot{x}^{4}}{\left(1+\dot{x}^{2}\right)^{3 / 2}}\right\} \psi d t= \\
& =-\int_{0}^{d} \frac{\dot{x}^{2}+x \ddot{x}+\dot{x}^{4}}{\left(1+\dot{x}^{2}\right)^{3 / 2}} \psi d t .
\end{aligned}
$$

Portanto, $A^{\prime}(0)=2 \pi \int_{0}^{d}\left\{\sqrt{1+\dot{x}^{2}}-\frac{\dot{x}^{2}+x \ddot{x}+\dot{x}^{4}}{\left(1+\dot{x}^{2}\right)^{3 / 2}}\right\} \psi d t$. Após alguns cálculos e por (5.6), concluímos finalmente que

$$
A^{\prime}(0)=2 \pi \int_{0}^{d} x\left\{\frac{1}{x\left(1+\dot{x}^{2}\right)^{1 / 2}}-\frac{\ddot{x}}{\left(1+\dot{x}^{2}\right)^{3 / 2}}\right\} \psi d t=2 \pi \int_{0}^{d} 2 H x(t) \psi(t) d t .
$$
temos:

Analogamente por (5.8), $V\left(x_{\epsilon}\right)=\pi \int_{0}^{d}(x+\epsilon \psi)^{2} d t$; derivando-se em relação a $\epsilon$,

$$
V^{\prime}(\epsilon):=\frac{d}{d \epsilon} V\left(x_{\epsilon}\right)=2 \pi \int_{0}^{d}(x+\epsilon \psi) \psi d t
$$


e tomando-se em $\epsilon=0$, resulta

$$
V^{\prime}(0)=2 \pi \int_{0}^{d} x(t) \psi(t) d t
$$

O próximo resultado garante que, sob certas condições, existe uma variação que preserva volume.

Lema 5.1.1. Seja $\psi \in C^{2}([0, d], \mathbb{R})$. Então, existe uma variação $x_{\epsilon}$ que preserva volume, $\epsilon \in]-\epsilon_{0}, \epsilon_{0}\left[\right.$, com $x_{0}=\left.x \quad e \frac{d}{d \epsilon} x_{\epsilon}\right|_{\epsilon=0}=\psi$ (isto é, com vetor-variação $\psi$ ), se e somente se $\int_{0}^{d} x(t) \psi(t) d t=0$.

Além disto, a condição acima equivale a $\int_{S} f d a=0$, ou seja, a componente normal $f$ do vetor variação, $f:=\frac{\psi}{\sqrt{1+\dot{x}^{2}}}$, tem valor médio nulo sobre sobre a superfície $S$, onde da denota o elemento de área de $S$.

Prova: Supomos que existe uma variação $\left.x_{\epsilon}:=x+\epsilon \psi, \epsilon \in\right]-\epsilon_{0}, \epsilon_{0}\left[, x_{0}=x, \quad \psi=\right.$ $\left.\frac{d}{d \epsilon} x_{\epsilon}\right|_{\epsilon=0}$, que preserva volume, ou seja, $V\left(x_{\epsilon}\right)=c$, onde $c \in \mathbb{R}$ é uma constante, conforme definimos na seção 1.1. Logo, $\delta V=\left.\frac{d}{d \epsilon} V\left(x_{\epsilon}\right)\right|_{\epsilon=0}=V^{\prime}(0)=0$.

Por (5.10) segue que $\int_{0}^{d} x(t) \psi(t) d t=0$.

Reciprocamente, seja $\psi \in C^{2}([0, d], \mathbb{R})$ satisfazendo $\int_{0}^{d} x(t) \psi(t) d t=0$.

Vamos mostrar que existe uma variação, nestas condições, que preserva volume. Consideramos uma variação de $x$ dada por $x(\epsilon, \delta)=x+\epsilon \psi+\delta \phi$, com $\phi \in C_{c}^{2}([0, d], \mathbb{R})$ (isto é, $\phi$ é de classe $C^{2}$ e tem suporte compacto ), satisfazendo $\int_{0}^{d} x(t) \phi(t) d t \neq 0$. Observamos que existe uma tal $\phi$ pois, caso contrário, teríamos $\int_{0}^{d} x(t) \phi(t) d t=0, \forall \phi \in C_{c}^{2}([0, d], \mathbb{R})$, e pelo Lema 1.1.2 isto implicaria que $x \equiv 0$, o que é absurdo, pois $x(t)>0$.

Denotamos agora por $V(\epsilon, \delta)$ o volume encerrado pela superfície de rotação gerada pela curva $x(\epsilon, \delta)$ e consideramos a equação

$$
V(\epsilon, \delta)=k
$$

onde $k \in \mathbb{R}$ é uma constante. Logo, procedendo de forma análoga a (5.10) e utilizando a 
hipótese, temos

$$
\left.\frac{\partial}{\partial \delta} V(\epsilon, \delta)\right|_{(0,0)}=\left.\int_{0}^{d}(x(t)+\epsilon \psi(t)+\delta \phi(t)) \phi(t)\right|_{(0,0)} d t=2 \pi \int_{0}^{d} x(t) \phi(t) d t \neq 0
$$

Pelo Teorema das funções implícitas, podemos tomar $\delta=\delta(\epsilon), \quad \delta \in C^{\infty}$, em uma vizinhança de $\epsilon=0$. Além disto, $\delta(0)=0$.

Definindo $x_{\epsilon}:=x(\epsilon, \delta)=x+\epsilon \psi+\delta(\epsilon) \phi$, temos que:

(i) $x_{\epsilon}$ preserva volume,

já que pelo Teorema das funções implícitas temos $V(\epsilon, \delta(\epsilon))=k$, onde $k \in \mathbb{R}$ é uma constante.

(ii) $x_{\epsilon}$ tem $\psi$ como vetor-variação.

Considerando a equação $V(\epsilon, \delta(\epsilon))=k$, segue que $\frac{\partial V}{\partial \epsilon}+\frac{\partial V}{\partial \delta} \frac{d \delta}{d \epsilon}=0$, e portanto, $\frac{\partial V}{\partial \epsilon}(0,0)+\frac{\partial V}{\partial \delta}(0,0) \frac{d \delta}{d \epsilon}(0)=0$.

Por (5.12), podemos dividir a igualdade acima por $\frac{\partial V}{\partial \delta}(0,0)$ e, procedendo analogamente como em (5.10) resulta, utilizando-se a hipótese, que:

$$
\frac{d \delta}{d \epsilon}(0)=-\left(\frac{\partial V}{\partial \epsilon}(0,0)\right) \cdot\left(\frac{\partial V}{\partial \delta}(0,0)\right)^{-1}=-\left(2 \pi \int_{0}^{d} x(t) \psi(t) d t\right) \cdot\left(2 \pi \int_{0}^{d} x(t) \phi(t) d t\right)=0 .
$$

Consequentemente,

$$
\left.\frac{d}{d \epsilon} x_{\epsilon}\right|_{\epsilon=0}=\left.\left(\psi+\frac{d \delta}{d \epsilon} \phi\right)\right|_{\epsilon=0}=\psi+\frac{d \delta}{d \epsilon}(0) \phi=\psi \text {. }
$$

Para terminar a prova, mostramos a equivalência $\int_{0}^{d} x(t) \psi(t) d t=0 \Longleftrightarrow \int_{S} f d a=$ 0 , onde $f:=\frac{\psi}{\sqrt{1+\dot{x}^{2}}}\left(\right.$ ver Lema 1.5.6) é a componente normal do vetor-variação $\psi$ de $x_{\epsilon}$. Como estamos tratando de superfícies rotacionais, utilizando a parametrização (5.1) segue que $d a=2 \pi x(t) \sqrt{1+\dot{x}^{2}} d t$. Logo,

$$
\int_{S} f d a=2 \pi \int_{0}^{d} \frac{\psi(t)}{\sqrt{1+\dot{x}^{2}(t)}} x(t) \sqrt{1+\dot{x}^{2}(t)} d t=2 \pi \int_{0}^{d} x(t) \psi(t) d t,
$$

o que implica na equivalência mencionada. 
Com o objetivo de aplicar o método dos multiplicadores de Lagrange ao nosso problema $(\mathrm{P})$, definimos a constante

$$
H_{0}:=\frac{2 \pi}{A(x)} \int_{0}^{d} H x \sqrt{1+\dot{x}^{2}} d t
$$

e o funcional $J:]-\epsilon_{0}, \epsilon_{0}\left[\rightarrow \mathbb{R}\right.$ dado por $J\left(x_{\epsilon}\right):=A\left(x_{\epsilon}\right)-2 H_{0} V\left(x_{\epsilon}\right)$.

Para simplificar, continuaremos usando os símbolos $J(\epsilon), A(\epsilon)$ e $V(\epsilon)$ no lugar de $J\left(x_{\epsilon}\right), A\left(x_{\epsilon}\right)$ e $V\left(x_{\epsilon}\right)$.

O próximo resultado nos garante que as superfícies de curvatura média constante que encontram os planos $P_{1}$ e $P_{2}$ perpendicularmente são pontos críticos do funcional área, para toda variação que preserva volume. Ainda assim, não garantimos que estas superfícies minimizam área.

Proposição 5.1.2. As seguintes afirmações são equivalentes:

(i) S tem curvatura média constante $H_{0}$ e encontra os planos $P_{1}$ e $P_{2}$ perpendicularmente ao longo de seu bordo $\partial S$;

(ii) $A^{\prime}(0)=0$, para cada variação que preserva volume $\left.x_{\epsilon}, \epsilon \in\right]-\epsilon_{0}, \epsilon_{0}\left[, x_{0}=x\right.$;

(iii) $J^{\prime}(0)=0$, para toda variação.

\section{Prova:}

(i) $\Longrightarrow$ (iii): Seja $x_{\epsilon}$ uma variação qualquer, $x_{\epsilon}:=x+\epsilon \psi, \operatorname{com} \psi=\left.\frac{d}{d \epsilon} x_{\epsilon}\right|_{\epsilon=0}$. Pela definição de $J$, temos que $J^{\prime}(\epsilon):=A^{\prime}(\epsilon)-2 H_{0} V^{\prime}(\epsilon)$.

Como S tem curvatura média constante $H=H_{0}$ e encontra os planos $P_{1}$ e $P_{2}$ perpendicularmente, segue então de (5.9) e (5.10) que:

$$
J^{\prime}(0)=A^{\prime}(0)-2 H_{0} V^{\prime}(0)=2 \pi\left\{2 H_{0} \int_{0}^{d} x(t) \psi(t) d t-2 H_{0} \int_{0}^{d} x(t) \psi(t) d t\right\}=0 .
$$

Como $x_{\epsilon}$ é qualquer, então obtemos (iii).

$($ iii $) \Longrightarrow($ ii $)$ : Seja $x_{\epsilon}$ uma variação qualquer que preserva volume, $\left.\epsilon \in\right]-\epsilon_{0}, \epsilon_{0}\left[, x_{0}=x\right.$, ou seja, $V(\epsilon)=V(0), \forall \epsilon \in]-\epsilon_{0}, \epsilon_{0}\left[\right.$ e portanto, $V^{\prime}(0)=0$. Pela hipótese (iii), vale para esta $x_{\epsilon}$ que $J^{\prime}(0)=0$. Com isto, segue da definição de $J$ que:

$J^{\prime}(0)=A^{\prime}(0)-2 H_{0} V^{\prime}(0)=A^{\prime}(0)$. 
Como $x_{\epsilon}$ é uma variação arbitrária que preserva volume, então obtemos (ii).

(ii) $\Longrightarrow($ i): Nos Teoremas 2.2.1 e 2.3.1 mostramos que se $\mathrm{S}$ for uma solução do problema (P) então $\mathrm{S}$ tem cuvatura média constante e encontra os planos $P_{1}$ e $P_{2}$ perpendicularmente ao longo de seu bordo. No entanto, observando as provas destes dois resultados, usamos apenas que $S$ é um ponto crítico do funcional área, não importando para a demonstração se $\mathrm{S}$ minimiza ou maximiza este funcional, isto é, não importou o sinal de $A^{\prime \prime}(0)$. Desta forma, temos que se $A^{\prime}(0)=0$, para toda variação que preserva volume, então obtemos (i).

No próximo resultado tratamos de uma equivalência para os mínimos relativos do funcional área.

Proposição 5.1.3. As seguintes afirmações são equivalentes:

(i) $A^{\prime \prime}(0) \geq 0$, para toda variação que preserva volume $\left.x_{\epsilon}, \epsilon \in\right]-\epsilon_{0}, \epsilon_{0}\left[, x_{0}=x\right.$;

(ii) $J^{\prime \prime}(0) \geq 0$, para toda variação $x_{\epsilon}$ cujo vetor-variação $\psi$ satisfaz $\int_{0}^{d} x(t) \psi(t) d t=0$ ( pelo Lema 5.1.1 isto equivale a $\int_{S} f d a=0$ ).

\section{Prova:}

$(i i) \Longrightarrow(i)$ : Seja $x_{\epsilon}$ uma variação qualquer que preserva volume, $\left.\epsilon \in\right]-\epsilon_{0}, \epsilon_{0}\left[, x_{0}=x\right.$, com vetor-variação $\psi$, isto é $\left.\frac{d}{d \epsilon} x_{\epsilon}\right|_{\epsilon=0}=\psi$. Pelo Lema 5.1.1, a condição $\int_{0}^{d} x(t) \psi(t) d t=0$ é satisfeita. Por hipótese, $J^{\prime \prime}(0) \geq 0$ para esta $x_{\epsilon}$. Por outro lado, como $x_{\epsilon}$ preserva volume então $V^{\prime \prime}(0)=0 \mathrm{e}$, usando a definição de $\mathrm{J}$ resulta que $J^{\prime}(0)=A^{\prime \prime}(0)-2 H_{0} V^{\prime \prime}(0)=A^{\prime \prime}(0)$.

Ou seja, $A^{\prime \prime}(0) \geq 0$. Como $x_{\epsilon}$ é uma variação arbitrária que preserva volume, então obtemos (i).

$(i) \Longrightarrow(i i)$ : Seja $x_{\epsilon}$ uma variação qualquer, cujo vetor-variação $\psi$ satisfaz $\int_{0}^{d} x(t) \psi(t) d t=$ 0. Pelo Lema 5.1.1, existe uma variação $y_{\epsilon}$ que preserva volume, $\psi=\left.\frac{d}{d \epsilon} y_{\epsilon}\right|_{\epsilon=0}$. Logo $x_{\epsilon}$ e $y_{\epsilon}$ têm $\psi$ como vetor-variação. No Lema 5.1 .6 a seguir, mostramos que a expressão de $J^{\prime \prime}(0)$ depende somente da componente normal $f$ do vetor-variação. Consequentemente, $J^{\prime \prime}(0)$ é o mesmo para $x_{\epsilon}$ e $y_{\epsilon}$, que denotamos por $J^{\prime \prime}(0)\left[x_{\epsilon}\right]=J^{\prime \prime}(0)\left[y_{\epsilon}\right]$. Como $y_{\epsilon}$ preserva volume então $V^{\prime \prime}(0)\left[y_{\epsilon}\right]=0$ e, por hipótese, $A^{\prime \prime}(0)\left[y_{\epsilon}\right] \geq 0$. Logo, pela definição 
de $\mathrm{J}, J^{\prime \prime}(0)\left[x_{\epsilon}\right]=J^{\prime \prime}(0)\left[y_{\epsilon}\right]=A^{\prime \prime}(0)\left[y_{\epsilon}\right]-2 H_{0} V^{\prime \prime}(0)\left[y_{\epsilon}\right]=A^{\prime \prime}(0)\left[y_{\epsilon}\right] \geq 0$. Como $x_{\epsilon}$ é uma variação qualquer, obtemos então (ii).

Apresentamos agora o conceito de estabilidade de uma superfície.

Definição 5.1.4. Dizemos que uma superfície $S$ é estável se ela for um mínimo relativo do problema variacional, isto é, $A^{\prime}(0)=0$ e a $2^{\underline{a}}$ variação $\delta^{2} A=A^{\prime \prime}(0)$ dos funcionais área $A(S)$ satisfaz $A^{\prime \prime}(0) \geq 0$, para todas variações que preservam volume. Além disto, se $S$ não é estável, dizemos que $S$ é instável.

Observação 5.1.5. Como estamos interessados em determinar as superfícies que minimizam o funcional área $A(S)$ sob a condição adicional que o volume $V(S)$ encerrado por elas é constante, então segue da definição acima que elas devem ser estáveis. Além disto, as Proposições 5.1 .2 e 5.1 .3 nos garantem que as soluções do problema $(P)$ são superfícies de curvatura média constante, que encontram os planos $P_{1}$ e $P_{2}$ perpendicularmente e satisfazem $J^{\prime \prime}(0) \geq 0$, para todas variações com $\int_{0}^{d} f d a=0$, onde $f$ denota a componente normal do vetor-variação.

Como já sabemos que as soluções de (P) são superfícies rotacionais, estaremos considerando variações rotacionalmente simétricas de $\mathrm{S}$ e obtemos, para elas, a expressão de $J^{\prime \prime}(0)$.

Lema 5.1.6. Seja $S$ uma superfície rotacional gerada pela curva $\gamma(t)=(x(t), t)$, $x(t)>0 t \in[0, d], d:=\operatorname{dist}\left(P_{1}, P_{2}\right)$, com curvatura média $H$ constante, que encontra os planos $P_{1}$ e $P_{2}$ perpendicularmente ao longo de seu bordo $\partial S$. Então, a $2^{a}$ variação dos funcionais $J(S)$, para variações rotacionalmente simétricas, é dada por

$$
\delta^{2} J=J^{\prime \prime}(0)=\int_{S}\left(\|\nabla f\|^{2}-\|B\|^{2} f^{2}\right) d a,
$$

onde $f$ denota a componente normal do vetor variação, $\nabla$ o gradiente, $\|B\|$ a norma da

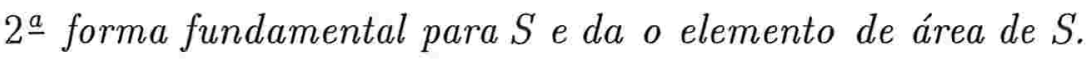

Prova: Como S é uma superfície rotacional gerada pela curva $\gamma(t)=(x(t), t)$, então podemos tomar (5.1) como sendo uma parametrização de S.

Consideramos variações $x_{\epsilon}$ da curva $\left.\gamma(t), \epsilon \in\right]-\epsilon_{0}, \epsilon_{0}\left[,\left.\operatorname{com} \frac{d}{d \epsilon} x_{\epsilon}\right|_{\epsilon=0}=\psi\right.$. 
Logo, a variação $x_{\epsilon}$ da curva $\gamma$ determina as seguintes variações da superfície $\mathrm{S}$

$$
\varphi_{\epsilon}(t, \phi):=\left(x_{\epsilon} \cos \phi, x_{\epsilon} \sin \phi, t\right)
$$

$\epsilon \in]-\epsilon_{0}, \epsilon_{0}\left[, \varphi_{0}=\varphi\right.$, onde $\varphi$ é dada em (5.1). Desta forma, temos que para cada $\epsilon \in]-\epsilon_{0}, \epsilon_{0}[$, (5.14) é uma variação rotacionalmente simétrica de S. Por (1.1) e (5.4), temos que a componente normal f do vetor- variação $\psi$ é dada por

$$
f=\frac{1}{\sqrt{1+\dot{x}^{2}}}\left(\left.\frac{d}{d \epsilon} x_{\epsilon}\right|_{\epsilon=0} \cos \phi,\left.\frac{d}{d \epsilon} x_{\epsilon}\right|_{\epsilon=0} \sin \phi, 0\right) \cdot(-\cos \phi,-\sin \phi, \dot{x}) \text {. }
$$

Como $\left.\frac{d}{d \epsilon} x_{\epsilon}\right|_{\epsilon=0}=\psi$, obtemos

$$
f(t, \phi)=\frac{1}{\sqrt{1+\dot{x}^{2}(t)}}(\psi \cos \phi, \psi \sin \phi, 0) \cdot(-\cos \phi,-\sin \phi, \dot{x}(t))=\frac{-\psi(t)}{\sqrt{1+\dot{x}^{2}(t)}} .
$$

Observamos que o sinal negativo apareceu na expressão de $f(t, \phi)$ devido à escolha de orientação para $\mathrm{N}(\mathrm{S})$ em $\mathbb{R}^{3}$. Tomando a orientação oposta para $\mathrm{N}(\mathrm{S})$, temos que

$$
f(t, \phi)=\frac{\psi(t)}{\sqrt{1+\dot{x}^{2}(t)}}
$$

Além disto, esta expressão nos diz que f não depende de $\phi$, ou seja $f(t, \phi)=f(t)$ e, portanto $\frac{\partial f}{\partial \phi}=f_{\phi}=0$. Logo, pelo Lema 1.4 .1 e pelas relações $(5.3)$, temos que o gradiente de $\mathrm{f}$ é dado por

$$
\nabla f=\frac{G f_{t}}{E G} \varphi_{t}=\frac{f_{t}}{E} \varphi_{t}=\frac{1}{E} \dot{f} \varphi_{t}
$$

onde $f_{t}=\frac{\partial f}{\partial t}=\dot{f}, \quad \varphi_{t}=\frac{\partial \varphi}{\partial t}$. Então,

$$
\|\nabla f\|^{2}=\nabla f \cdot \nabla f=\frac{1}{E^{2}} \dot{f}^{2} \varphi_{t} \cdot \varphi_{t}=\frac{1}{E^{2}} \dot{f}^{2} E=\frac{1}{E} \dot{f}^{2}(t) .
$$

Novamente, temos por (5.3) que

$$
\|\nabla f\|^{2}=\frac{1}{1+\dot{x}^{2}} \dot{f}^{2} .
$$

Por (5.15), temos

$$
\begin{gathered}
\dot{f}(t)=\frac{\dot{\psi}}{\left(1+\dot{x}^{2}\right)^{1 / 2}}-\frac{\psi \dot{x} \ddot{x}}{\left(1+\dot{x}^{2}\right)^{3 / 2}} \text { e, consequentemente, } \\
\dot{f}^{2}(t)=\frac{\dot{\psi}^{2}}{1+\dot{x}^{2}}+\frac{\psi^{2} \dot{x}^{2} \ddot{x}^{2}}{\left(1+\dot{x}^{2}\right)^{3}}-\frac{2 \psi \dot{\psi} \dot{x} \ddot{x}}{\left(1+\dot{x}^{2}\right)^{2}} .
\end{gathered}
$$


Pelo Lema 1.4.2 e pelas relações (5.5), segue que

$$
\|B\|^{2}=\frac{\ddot{x}^{2}}{\left(1+\dot{x}^{2}\right)^{3}}+\frac{1}{x^{2}\left(1+\dot{x}^{2}\right)} .
$$

Por (5.15), (5.16), (5.17), (5.18) e sabendo que o elemento de área de $\mathrm{S}$ é dado por $d a=2 \pi x \sqrt{1+\dot{x}^{2}} d t$, obtemos por um lado, que:

$$
\begin{aligned}
& \int_{S}\left(\|\nabla f\|^{2}-\|B\|^{2} f^{2}\right) d a= \\
= & 2 \pi \int_{0}^{d} \frac{x}{\left(1+\dot{x}^{2}\right)^{1 / 2}}\left\{\frac{\dot{\psi}^{2}}{1+\dot{x}^{2}}+\frac{\psi^{2} \dot{x}^{2} \ddot{x}^{2}}{\left(1+\dot{x}^{2}\right)^{3}}-\frac{2 \psi \dot{\psi} \dot{x} \ddot{x}}{\left(1+\dot{x}^{2}\right)^{2}}-\frac{\psi^{2} \ddot{x}^{2}}{\left(1+\dot{x}^{2}\right)^{3}}-\frac{\psi^{2}}{x^{2}\left(1+\dot{x}^{2}\right)}\right\} d t \\
= & 2 \pi \int_{0}^{d}\left\{\frac{x}{\left(1+\dot{x}^{2}\right)^{1 / 2}}\left[\frac{\dot{\psi}^{2}}{1+\dot{x}^{2}}+\frac{\psi^{2} \ddot{x}^{2}}{\left(1+\dot{x}^{2}\right)^{3}}\left(\dot{x}^{2}-1\right)-\frac{2 \psi \dot{\psi} \dot{x}}{\left(1+\dot{x}^{2}\right)^{2}}\right]-\frac{\psi^{2}}{x\left(1+\dot{x}^{2}\right)^{3 / 2}}\right\} d t \\
= & 2 \pi \int_{0}^{d}\left\{\frac{x}{\left(1+\dot{x}^{2}\right)^{1 / 2}}\left[\frac{\dot{\psi}^{2}}{1+\dot{x}^{2}}+\frac{\psi^{2} \ddot{x}^{2}}{\left(1+\dot{x}^{2}\right)^{3}}\left(\dot{x}^{2}-1\right)-\frac{2 \psi \dot{\psi} \dot{x} \ddot{x}}{\left(1+\dot{x}^{2}\right)^{2}}+\frac{\psi^{2} \dot{x}^{2}}{x^{2}\left(1+\dot{x}^{2}\right)}\right]-k_{2} \psi^{2}\right\} d t,
\end{aligned}
$$

pela expressão de $k_{2}$ dada em (5.5).

Por hipótese, $\mathrm{S}$ tem curvatura média constante $H=\frac{1}{2}\left(k_{1}+k_{2}\right)$ e encontra os planos $P_{1}$ e $P_{2}$ perpendicularmente, ou seja, $\dot{x}(0)=\dot{x}(d)=0$ ( conforme (5.2)). Usando a expressão de $k_{1}$, dada em (5.5), e fazendo-se a integração por partes do $3^{\underline{0}}$ termo da integral anterior, obtemos

$$
\begin{aligned}
& -\int_{0}^{d} \frac{2 \psi \dot{\psi} x \dot{x} \ddot{x}}{\left(1+\dot{x}^{2}\right)^{5 / 2}} d t=\int_{0}^{d} \frac{2 \psi \dot{\psi} x \dot{x}}{1+\dot{x}^{2}} k_{1} d t=\int_{0}^{d} 2 \psi \dot{\psi} \frac{x \dot{x}}{1+\dot{x}^{2}}\left(2 H-k_{2}\right) d t= \\
& =\left.\left\{\psi^{2} \frac{x \dot{x}}{1+\dot{x}^{2}}\left(2 H-k_{2}\right)\right\}\right|_{0} ^{d}- \\
& -\int_{0}^{d} \psi^{2}\left\{\frac{\dot{x}^{2}}{1+\dot{x}^{2}}\left(2 H-k_{2}\right)+\frac{x \dot{x}}{1+\dot{x}^{2}}\left(2 H-k_{2}\right)-\frac{2 x \dot{x}^{2} \ddot{x}}{\left(1+\dot{x}^{2}\right)^{2}}\left(2 H-k_{2}\right)-\frac{x \dot{x}}{1+\dot{x}^{2}} \dot{k}_{2}\right\} d t .
\end{aligned}
$$

Agora usamos que $\dot{x}(0)=\dot{x}(d)=0,2 H-k_{2}=k_{1}=-\frac{\ddot{x}}{\left(1+\dot{x}^{2}\right)^{3 / 2}} \mathrm{e}$ $\dot{k}_{2}=-\frac{\dot{x}}{x^{2}\left(1+\dot{x}^{2}\right)^{1 / 2}}-\frac{x \ddot{x}}{x\left(1+\dot{x}^{2}\right)^{3 / 2}}$.

Logo, $-\int_{0}^{d} \frac{2 \psi \dot{\psi} x \dot{x} \ddot{x}}{\left(1+\dot{x}^{2}\right)^{5 / 2}} d t=\int_{0}^{d} \psi^{2}\left\{\frac{\dot{x}^{2} \ddot{x}}{\left(1+\dot{x}^{2}\right)^{5 / 2}}+\frac{x \ddot{x}^{2}\left(1-\dot{x}^{2}\right)}{\left(1+\dot{x}^{2}\right)^{7 / 2}}-\frac{x \dot{x}^{2}}{x^{2}\left(1+\dot{x}^{2}\right)^{3 / 2}}-\frac{x \dot{x}^{2} \ddot{x}}{x\left(1+\dot{x}^{2}\right)^{5 / 2}}\right\} d t$. 
Assim,

$$
\begin{aligned}
& \int_{S}\left(\|\nabla f\|^{2}-\|B\|^{2} f^{2}\right) d a= \\
& =2 \pi \int_{0}^{d}\left\{\frac{x}{\left(1+\dot{x}^{2}\right)^{1 / 2}}\left[\frac{\dot{\psi}^{2}}{1+\dot{x}^{2}}+\frac{\psi^{2} \ddot{x}^{2}}{\left(1+\dot{x}^{2}\right)^{3}}\left(\dot{x}^{2}-1\right)+\frac{\psi^{2} \dot{x}^{2}}{x^{2}\left(1+\dot{x}^{2}\right)}\right]-k_{2} \psi^{2}\right\} d t+ \\
& +2 \pi \int_{0}^{d} \psi^{2}\left\{\frac{\dot{x}^{2} \ddot{x}}{\left(1+\dot{x}^{2}\right)^{5 / 2}}+\frac{x \ddot{x}^{2}\left(1-\dot{x}^{2}\right)}{\left(1+\dot{x}^{2}\right)^{7 / 2}}-\frac{x \dot{x}^{2}}{x^{2}\left(1+\dot{x}^{2}\right)^{3 / 2}}-\frac{\dot{x}^{2}}{\left(1+\dot{x}^{2}\right)^{5 / 2}}\right\} d t
\end{aligned}
$$

Ou seja,

$$
\int_{S}\left(\|\nabla f\|^{2}-\|B\|^{2} f^{2}\right) d a=2 \pi \int_{0}^{d}\left\{\frac{\dot{\psi}^{2} x}{\left(1+\dot{x}^{2}\right)^{3 / 2}}-k_{2} \psi^{2}\right\} d t .
$$

Por outro lado, vamos obter a expressão de $J^{\prime \prime}(0)$, usando que $J^{\prime \prime}(0)=A^{\prime \prime}(0)-2 H V^{\prime \prime}(0)$, onde $H=H_{0}$.

Denotamos por $\psi_{\epsilon}=\frac{d}{d \epsilon} x_{\epsilon}, \quad \psi_{0}=\psi$ e $\psi^{\prime}=\left.\frac{d}{d \epsilon} \psi_{\epsilon}\right|_{\epsilon=0}$. Então, como $A(x)=2 \pi \int_{0}^{d} x \sqrt{1+\dot{x}^{2}} d t$, segue que

$$
\begin{aligned}
& A\left(x_{\epsilon}\right)=2 \pi \int_{0}^{d} x_{\epsilon} \sqrt{1+\dot{x}_{\epsilon}^{2}} d t \text { e, consequentemente } \\
& A^{\prime}(\epsilon):=\frac{d}{d \epsilon} A\left(x_{\epsilon}\right)=2 \pi \int_{0}^{d}\left\{\psi_{\epsilon} \sqrt{1+\dot{x}_{\epsilon}^{2}}+\frac{x_{\epsilon} \dot{x_{\epsilon}} \dot{\psi}_{\epsilon}}{\left(1+\dot{x}_{\epsilon}^{2}\right)^{1 / 2}}\right\} d t .
\end{aligned}
$$

Portanto,

$$
\begin{aligned}
& \delta^{2} A=A^{\prime \prime}(0)=\left.\frac{d^{2}}{d \epsilon^{2}} A\left(x_{\epsilon}\right)\right|_{\epsilon=0}=\left.2 \pi \int_{0}^{d} \frac{d}{d \epsilon}\left\{\psi_{\epsilon} \sqrt{1+\dot{x}_{\epsilon}^{2}}+\frac{x_{\epsilon} \dot{x}_{\epsilon} \dot{\psi}_{\epsilon}}{\left(1+\dot{x}_{\epsilon}^{2}\right)^{1 / 2}}\right\}\right|_{\epsilon=0} d t= \\
& =2 \pi \int_{0}^{d}\left\{\psi^{\prime} \sqrt{1+\dot{x}^{2}}+\frac{2 \dot{x} \psi \dot{\psi}}{\left(1+\dot{x}^{2}\right)^{1 / 2}}+\frac{x \dot{\psi}^{2}}{\left(1+\dot{x}^{2}\right)^{1 / 2}}+\frac{x \dot{x} \dot{\psi}^{\prime}}{\left(1+\dot{x}^{2}\right)^{1 / 2}}-\frac{x \dot{x}^{2} \dot{\psi}^{2}}{\left(1+\dot{x}^{2}\right)^{3 / 2}}\right\} d t .
\end{aligned}
$$

Mas, observamos que

$$
\begin{aligned}
& \int_{0}^{d} \frac{x \dot{x}}{\left(1+\dot{x}^{2}\right)^{1 / 2}} \dot{\psi}^{\prime} d t=\left.\left\{\frac{x \dot{x}}{\left(1+\dot{x}^{2}\right)^{1 / 2}} \psi^{\prime}\right\}\right|_{0} ^{d}-\int_{0}^{d} \psi^{\prime} \frac{d}{d t}\left(\frac{x \dot{x}}{\left(1+\dot{x}^{2}\right)^{1 / 2}}\right) d t=-\int_{0}^{d} \psi^{\prime} \frac{d}{d t}\left(\frac{x \dot{x}}{\left(1+\dot{x}^{2}\right)^{1 / 2}}\right) d t \\
& \text { pois } \dot{x}(0)=\dot{x}(d)=0 .
\end{aligned}
$$

e

$$
\int_{0}^{d} 2 \psi \dot{\psi} \frac{\dot{x}}{\left(1+\dot{x}^{2}\right)^{1 / 2}} d t=\left.\left\{\psi^{2} \frac{\dot{x}}{\left(1+\dot{x}^{2}\right)^{1 / 2}}\right\}\right|_{0} ^{d}-\int_{0}^{d} \psi^{2} \frac{\ddot{x}}{\left(1+\dot{x}^{2}\right)^{3 / 2}} d t=\int_{0}^{d} \psi^{2} k_{1} d t
$$


onde usamos que $\dot{x}(0)=\dot{x}(d)=0$ e $(5.5)$.

Logo,

$$
A^{\prime \prime}(0)=2 \pi \int_{0}^{d}\left\{\psi^{\prime}\left[\left(1+\dot{x}^{2}\right)^{1 / 2}-\frac{d}{d t}\left(\frac{x \dot{x}}{\left(1+\dot{x}^{2}\right)^{1 / 2}}\right)\right]+\left[\psi^{2} k_{1}+\frac{x \dot{\psi}^{2}}{\left(1+\dot{x}^{2}\right)^{3 / 2}}\right]\right\} d t
$$

onde $\frac{d}{d t}\left(\frac{x \dot{x}}{\left(1+\dot{x}^{2}\right)^{1 / 2}}\right)=\frac{\left(1+\dot{x}^{2}\right) \dot{x}^{2}+x \ddot{x}}{\left(1+\dot{x}^{2}\right)^{3 / 2}}$.

Procedendo de forma análoga a $A(x)$, temos que $V(x)=\pi \int_{0}^{d} x^{2}(t) d t$ e portanto, $V\left(x_{\epsilon}\right)=\pi \int_{0}^{d} x_{\epsilon}^{2}(t) d t$ e, consequentemente, $V^{\prime}(\epsilon)=2 \pi \int_{0}^{d} x_{\epsilon} \psi_{\epsilon} d t$.

Portanto,

$$
\delta^{2} V=V^{\prime \prime}(0)=\frac{d^{2}}{d \epsilon^{2}} V\left(x_{\epsilon}\right)=2 \pi \int_{0}^{d}\left\{\psi^{2}+x \psi^{\prime}\right\} d t
$$

Juntando todos os cálculos anteriores, concluímos que:

$$
\begin{aligned}
& \delta^{2} J=J^{\prime \prime}(0)=A^{\prime \prime}(0)-\left(k_{1}+k_{2}\right) V^{\prime \prime}(0)= \\
& =2 \pi \int_{0}^{d}\left\{\psi^{\prime}\left[\left(1+\dot{x}^{2}\right)^{1 / 2}-\frac{d}{d t}\left(\frac{x \dot{x}}{\left(1+\dot{x}^{2}\right)^{1 / 2}}\right)\right]+\left[\psi^{2} k_{1}+\frac{x \dot{\psi}^{2}}{\left(1+\dot{x}^{2}\right)^{3 / 2}}\right]-k_{1} \psi^{2}-k_{1} x \psi^{\prime}-\right. \\
& \left.-k_{2} \psi^{2}-k_{2} x \psi^{\prime}\right\} d t \\
& =2 \pi \int_{0}^{d}\left\{\frac{x \dot{\psi}^{2}}{\left(1+\dot{x}^{2}\right)^{3 / 2}}-k_{2} \psi^{2}+\psi^{\prime}\left[-2 H x+\left(1+\dot{x}^{2}\right)^{1 / 2}-\frac{d}{d t}\left(\frac{x \dot{x}}{\left(1+\dot{x}^{2}\right)^{1 / 2}}\right)\right]\right\} d t .
\end{aligned}
$$

No entanto, obtemos

$$
\left(1+\dot{x}^{2}\right)^{1 / 2}-\frac{d}{d t}\left(\frac{x \dot{x}}{\left(1+\dot{x}^{2}\right)^{1 / 2}}\right)=x\left(\frac{1}{x\left(1+\dot{x}^{2}\right)^{1 / 2}}-\frac{\ddot{x}}{\left(1+\dot{x}^{2}\right)^{1 / 2}}\right)=2 H x, \text { devido a (5.6). }
$$

Portanto,

$$
\delta^{2} J=2 \pi \int_{0}^{d}\left\{\frac{x \dot{\psi}^{2}}{\left(1+\dot{x}^{2}\right)^{3 / 2}}-k_{2} \psi^{2}\right\} d t
$$

Comparando (5.19) e (5.20), resulta que

$$
\delta^{2} J=J^{\prime \prime}(0)=\int_{S}\left(\|\nabla f\|^{2}-\|B\|^{2} f^{2}\right) d a .
$$

Passemos agora a estudar a estabilidade de cada uma das possíveis soluções do problema variacional $(\mathrm{P})$ : o cilindro, a semiesfera e o ondulóide. Como estas superfícies têm 
curvatura média constante e supomos que elas encontam os planos $P_{1}$ e $P_{2}$ perpendicularmente, temos pela Proposição 5.1.2 que elas satisfazem $A^{\prime}(0)=0$, para variações que preservam volume. Para estudarmos o critério de estabilidade para elas, basta então analisar o sinal de $J^{\prime \prime}(0)$, para variações que satisfazem $\int_{S} f a=0$ ( conforme Proposição $5.1 .3)$.

\subsection{A instabilidade / estabilidade do cilindro}

Consideramos a superfície rotacional $\mathrm{S}$ como sendo um cilindro de raio $\rho$ e, portanto, a parametrização (5.1) de S se torna:

$$
\varphi(t, \phi)=(\rho \cos \phi, \rho \sin \phi, t)
$$

$t \in[0, d], d:=\operatorname{dist}\left(P_{1}, P_{2}\right), \quad \phi \in\left[0,2 \pi\left[, \rho \in \mathbb{R}_{+}^{*}\right.\right.$.

Temos então o seguinte resultado:

Teorema 5.2.1. Se a superfície $S$ é um cilindro de raio $\rho$, então $S$ é instável para $\pi \rho<$, onde $d:=\operatorname{dist}\left(P_{1}, P_{2}\right)$.

Prova: Considerando a parametrização (5.21) do cilindro, temos pelas relações (5.3), (5.5) e pelo Lema 1.4.2 que:

$$
\begin{aligned}
& E=1+\dot{x}^{2}=1, \text { visto que } x(t)=\rho \text { e, portanto } \dot{x}=0 . \\
& k_{1}=\frac{-\ddot{x}}{\left(1+\dot{x}^{2}\right)^{3 / 2}}=0, \quad k_{2}=\frac{1}{x \sqrt{1+\dot{x}^{2}}}=\frac{1}{\rho}, \quad\|B\|^{2}=k_{1}^{2}+k_{2}^{2}=\frac{1}{\rho^{2}} .
\end{aligned}
$$

Além disto, o elemento de área do cilindro é $d a=2 \pi x \sqrt{1+\dot{x}^{2}} d t=\rho d t$. Por (5.13), a expressão de $J^{\prime \prime}(0)$ para o cilindro é então dada por:

$$
\delta^{2} J=J^{\prime \prime}(0)=2 \pi \int_{0}^{d}\left(\dot{f}^{2}(t)-\frac{1}{\rho^{2}} f^{2}(t)\right) \rho d t .
$$

A seguir, buscaremos condições sobre a função f para que o cilindro seja instável. Pela. Definição 5.1.4, pelo Lema 5.1.1 e pela Proposição 5.1.3, o cilindro é instável se pudermos encontrar uma variação rotacionalmente simétrica da superfície, cuja componente normal f satisfaça a condição do valor médio nulo

$$
\int_{0}^{d} f(t) d t=0
$$


e tal que $\delta^{2} J<0$. Por (5.22), a instabilidade do cilindro equivale a $\rho^{2} \frac{\int_{0}^{d} \dot{f}^{2}(t) d t}{\int_{0}^{d} f^{2}(t) d t}<1$ para variações com $\int_{0}^{d} f(t) d t=0$.

Para simplificar, parametrizamos o cilindro no intervalo $[0,1]$ e temos:

$$
\tilde{\varphi}(t, \phi)=(\rho \cos \phi, \rho \sin \phi, t d),
$$

$t \in[0,1], \quad \phi \in[0,2 \pi[$.

Desta forma, a expressão de $\delta^{2} J$ para o cilindro se torna:

$$
\delta^{2} J=2 \pi \int_{0}^{1}\left(\frac{1}{d^{2}} \dot{f}^{2}(t)-\frac{1}{\rho^{2}} f^{2}(t)\right) \rho d d t .
$$

Como estamos interessados em $\delta^{2} J<0$, então por (5.24) e por $\rho d>0$, obtemos

$$
\rho^{2} \frac{1}{d^{2}} \frac{\int_{0}^{1} \dot{f}^{2}(t) d t}{\int_{0}^{1} f^{2}(t) d t}<1
$$

Portanto, a fim de obtermos para qual relação o cilindro é instável, procuramos $\mathrm{f}$ tal que

$$
\rho^{2} \frac{1}{d^{2}} \frac{\int_{0}^{1} \dot{f}^{2}(t) d t}{\int_{0}^{1} f^{2}(t) d t}<1
$$

sob a condição adicional $\int_{0}^{1} f(t) d t=0$.

No entanto, isto nos conduz ao seguinte problema variacional:

" queremos minimizar $\rho^{2} \frac{1}{d^{2}} \frac{\int_{0}^{1} \dot{f}^{2}(t) d t}{\int_{0}^{1} f^{2}(t) d t}$ com a condição adicional $\int_{0}^{1} f(t) d t=0$. Além disto, queremos que o mínimo da quantidade acima seja menor que 1" .

Observamos agora que se tomarmos $\mathrm{E}$ como o espaço das funções e definirmos aplicações $\alpha, \beta: E \rightarrow \mathbb{R}$ por

$$
\alpha(f)=\int_{0}^{1} \dot{f}^{2}(t) d t \quad \text { e } \quad \beta(f)=\int_{0}^{1} f^{2}(t) d t, \quad \forall f \in E, \quad \text { então : }
$$


- se $f \neq 0$, segue que $\beta(f)>0$

- $\forall c>0, \alpha(c f)=c^{2} \alpha(f)$ e $\beta(c f)=c^{2} \beta(f)$.

Portanto, como queremos obter variações com $f \neq 0$, segue do Lema 1.4.3 que o problema variacional acima equivale a " minimizar $\int_{0}^{1} \dot{f}^{2}(t) d t$ sob as condições adicionais $\int_{0}^{1} f^{2}(t) d t=1$ e $\int_{0}^{1} f(t) d t=0$, notando ainda que $\rho^{2} \frac{1}{d^{2}}$ é uma constante.

Como procuramos por condições necessárias que f deve satisfazer para que seja solução do problema anterior, então basta obter a equação de Euler - Lagrange com vínculos para este problema. Com base no método dos multiplicadores de Lagrange (Lema 1.1.4 ), temos que existem constantes reais $\lambda, \mu$ tais que o Lagrangeano $\mathcal{L}$ é dado por

$$
\mathcal{L}=\int_{0}^{1}\left(\dot{f}^{2}(t)-\lambda f^{2}(t)-\mu f(t)\right) d t
$$

Seja $f_{\epsilon}$ uma variação rotacionalmente simétrica de $\left.f, \epsilon \in\right]-\epsilon_{0}, \epsilon_{0}\left[, f_{0}=f\right.$, $\left.\frac{d}{d \epsilon} f_{\epsilon}\right|_{\epsilon=0}:=v$. Para que f seja um mínimo do problema variacional, é necessário que ela seja um ponto crítico de $\mathcal{L}_{\epsilon}$, onde $\mathcal{L}_{\epsilon}(f)=\int_{0}^{1}\left\{\dot{f}_{\epsilon}^{2}(t)-\lambda f_{\epsilon}^{2}(t)-\mu f_{\epsilon}(t)\right\} d t$, ou seja,

$$
0=\left.\frac{d}{d \epsilon} \mathcal{L}_{\epsilon}(f)\right|_{\epsilon=0}=\int_{0}^{1}\{2 \dot{f}(t) \dot{v}(t)-2 \lambda f(t) v(t)-\mu v(t)\} d t
$$

visto que $f \in C^{2}$ e, portanto, $\left.\frac{d}{d \epsilon} \frac{d}{d t} f_{\epsilon}(t)\right|_{\epsilon=0}=\left.\frac{d}{d t} \frac{d}{d \epsilon} f_{\epsilon}(t)\right|_{\epsilon=0}=\frac{d}{d t} v(t)=\dot{v}(t)$.

Integrando por partes o $1^{\circ}$ termo da integral (5.27), resulta que (5.27) pode ser reescrita como

$$
\left.\{2 \dot{f}(t) v(t)\}\right|_{0} ^{1}+\int_{0}^{1}\{-2 \ddot{f}(t) v(t)-2 \lambda f(t) v(t)-\mu v(t)\} d t=0, \forall v(t) .
$$

Em particular, tomando $v(t)$ em (5.28) com $v(1)=v(0)=0$, temos que $\int_{0}^{1}\{-2 \ddot{f}(t)-2 \lambda f(t)-\mu\} v(t) d t=0, \forall v(t) \operatorname{com} v(1)=v(0)=0$.

Pelo Lema 1.1.2, segue $-2 \ddot{f}(t)-2 \lambda f(t)-\mu=0, \forall t \in[0,1]$, ou seja,

$$
\ddot{f}(t)+\lambda f(t)=-\mu / 2, \forall t \in[0,1]
$$


Substituindo (5.29) em (5.28), segue que $\left.\{2 \dot{f}(t) v(t)\}\right|_{0} ^{1}=0, \forall v(t)$. Em particular, tomando $v(t)$ com $v(1) \neq 0$ e $v(0)=0$, resulta que $\dot{f}(1)=0$, e tomando $v(t)$ com $v(1)=0$ e $v(0) \neq 0$, resulta que $\dot{f}(0)=0$.

Portanto, obtemos a seguinte equação de Euler - Lagrange:

$$
\left\{\begin{array}{l}
\ddot{f}(t)+\lambda f(t)=-\mu / 2 \\
\dot{f}(0)=\dot{f}(1)=0
\end{array}\right.
$$

Usando a hipótese de que $\int_{0}^{1} f(t) d t=0$ na integração da $1^{\underline{a}}$ equação de $(5.30)$, temos que:

$\int_{0}^{1} \ddot{f}(t) d t=-\frac{\mu}{2} \int_{0}^{1} d t=-\frac{\mu}{2}$

No entanto, $\int_{0}^{1} \ddot{f}(t) d t=\dot{f}(1)-\dot{f}(0)=0$, por (5.30). Consequentemente $\mu=0$ e a equação de Euler - Lagrange (5.30) é, na verdade,

$$
\left\{\begin{array}{l}
\ddot{f}(t)+\lambda f(t)=0 \\
\dot{f}(0)=\dot{f}(1)=0
\end{array}\right.
$$

Lembramos que, de acordo com o Teorema 2.2.1, tínhamos que o multiplicador de Lagrange $\lambda$ e a curvatura média $H$ estavam relacionadas por $\lambda=2 H$. No caso do cilindro, temos $\lambda>0$, pois tomando a parametrização (5.21) do cilindro, temos que

$H=\frac{1}{2}\left(k_{1}+k_{2}\right)=\frac{1}{2 \rho}>0$. Resolvendo a equação diferencial ordinária (5.31), dadas as condições iniciais, temos que as soluções são da forma

$$
f_{\nu}(t)=\cos \left(\sqrt{\lambda_{\nu}} t\right) \quad \text { com } \quad \lambda_{\nu}=\nu^{2} \pi^{2}, \quad \nu=1,2,3, \ldots ; t \in[0,1] .
$$

Multiplicando a $1^{\underline{a}}$ equação de $(5.31)$ por $f(t)$, então:

$f(t) \ddot{f}(t)=-\lambda f^{2}(t)$,

e, integrando esta igualdade de 0 a 1 , temos:

$$
\int_{0}^{1} f(t) \ddot{f}(t) d t=-\lambda \int_{0}^{1} f^{2}(t) d t
$$

No entanto, integrando por partes e usando a segunda equação de (5.31), resulta que que:

$$
\int_{0}^{1} f(t) \ddot{f}(t) d t=\left.\{f(t) \dot{f}(t)\}\right|_{0} ^{1}-\int_{0}^{1} \dot{f}^{2}(t) d t=-\int_{0}^{1} \dot{f}^{2}(t) d t
$$


Portanto,

$$
\int_{0}^{1} \dot{f}^{2}(t) d t=\lambda \int_{0}^{1} f^{2}(t) d t .
$$

Temos que $\lambda$ é um autovalor associado à função $f(t)$ (solução de (5.31)), de onde segue que $\lambda_{\min }=\min _{\nu \in \mathbb{N}}\left\{\lambda_{\nu}\right\}=\lambda_{1}=\pi^{2} \leq \lambda$. Lembramos que o problema variacional, introduzido nesta seção, exigia ainda que o mínimo fosse menor do que 1 . Portanto, por (5.32), queremos que

$$
\frac{\rho^{2}}{d^{2}} \pi^{2} \leq \frac{\rho^{2}}{d^{2}} \lambda=\rho^{2} \frac{1}{d^{2}} \frac{\int_{0}^{1} \dot{f}^{2}(t) d t}{\int_{0}^{1} f^{2}(t) d t}<1 .
$$

Consequentemente, a instabilidade do cilindro ocorre para a relação $\pi \rho<d$. Ou seja, o cilindro é estável para $\pi \rho \geq d$ e, com isto, é um mínimo relativo do problema variacional $(\mathrm{P})$.

\subsection{Estabilidade da semiesfera}

Consideramos a superfície rotacional $\mathrm{S}$ como sendo uma semiesfera de raio r, com bordo $\partial S$ contido no plano $P_{1}$. Como S está localizada entre os planos $P_{1}$ e $P_{2}$ e $d:=\operatorname{dist}\left(P_{1}, P_{2}\right)$, então a relação $r<d$ deve ser satisfeita.

Usaremos o fato de que uma esfera é sempre estável, cuja prova pode ser encontrada em [4], a fim de provarmos a estabilidade da semiesfera.

Teorema 5.3.1. Se a superfície $S$ é uma semiesfera de raio $r, r$ i d, então $S$ é estável.

Prova: Refletimos a semiesfera $\mathrm{S}$ com respeito ao plano $P_{1}$. Denotamos por $S_{r}$ a imagem de S pela reflexão, e por $S^{\prime}$ a esfera obtida juntando-se S e $S_{r}$, isto é, $S^{\prime}=S \bigcup S_{r}$. Tomando um campo variacional para $\mathrm{S}$, cuja componente normal é dada por $\mathrm{f}$, e refletindoo em relação ao plano $P_{1}$, obtemos um campo variacional $V$ rotacionalmente simétrico para $S^{\prime}$, com componente normal também dada por $\mathrm{f}$.

Como, por hipótese, $\mathrm{S}$ encontra $P_{1}$ perpedicularmente ao longo de seu bordo $\partial S$, temos pela Definição 2.3.2 que a normal $\mathrm{N}$ à superfície $\mathrm{S}$, ao longo de $\partial S$, pertence a.o 
plano $P_{1}$. Além disto, como a componente normal do vetor-variação para $\mathrm{S}$ é definida por $f=\left.\frac{d}{d \epsilon} \varphi_{\epsilon}\right|_{\epsilon=0} \cdot N$, obtemos que o campo variacional $\mathrm{V}$ ao longo de $\partial S$ está contido em $P_{1}$.

Desta forma, quando refletimos $\mathrm{V}$ em relação a $P_{1}$, ele permanece fixado ao longo de $\partial S$, ou seja, a variação do campo variacional $\mathrm{V}$, ao longo de $\partial S$, é nula.

Observando que $S^{\prime}=S \bigcup S_{r}$ e $S \bigcap S_{r}=\partial S$, então a expressão da $2^{\underline{a}}$ variação para $S^{\prime}$ (conforme (5.13) ) para o campo variacional $\mathrm{V}$ rotacionalmente simétrico é dada por

$$
\begin{aligned}
& \delta^{2} J\left(S^{\prime}\right)=\delta^{2} J\left(S \cup S_{r}\right)= \\
& =\int_{S}\left(\|\nabla f\|^{2}-\|B\|^{2} f^{2}\right) d a+\int_{S_{r}}\left(\|\nabla f\|^{2}-\|B\|^{2} f^{2}\right) d a-\int_{\partial S}\left(\|\nabla f\|^{2}-\|B\|^{2} f^{2}\right) d a \\
& =2 \int_{S}\left(\|\nabla f\|^{2}-\|B\|^{2} f^{2}\right) d a=2 \delta^{2} J(S), \text { isto é }
\end{aligned}
$$

$$
\delta^{2} J\left(S^{\prime}\right)=2 \delta^{2} J(S)
$$

visto que $\int_{S \cap S_{r}}\left(\|\nabla f\|^{2}-\|B\|^{2} f^{2}\right) d a=\int_{\partial S}\left(\|\nabla f\|^{2}-\|B\|^{2} f^{2}\right) d a=0$, conforme a observação acima, e $\int_{S}\left(\|\nabla f\|^{2}-\|B\|^{2} f^{2}\right) d a=\int_{S_{r}}\left(\|\nabla f\|^{2}-\|B\|^{2} f^{2}\right) d a$.

Logo, se a semiesfera $\mathrm{S}$ fosse instável, ou seja, se existisse $\mathrm{f}$ com $\int_{S} f d a=0 \mathrm{e}$ $\delta^{2} J(S)<0$, então por (5.33) existiria também f com $\int_{S^{\prime}} f d a=0$ e $\delta^{2} J\left(S^{\prime}\right)<0$, o que implicaria que a esfera $S^{\prime}$ seria instável. Mas, isto seria uma contradição com o fato de que a esfera é estável. Portanto, provamos que uma semiesfera é sempre estável e, então, é um mínimo relativo para o problema $(\mathrm{P})$.

\subsection{Instabilidade do ondulóide}

Com base nos comentários acerca do ondulóide, feitos na introdução deste capítulo, vemos que basta considerar um semiperíodo do ondulóide ( que é obtido quando o parâmetro t da parametrização da elipse, dada em (ii) no Teorema 4.4.1, varia de 0 a $\pi$, isto é, $t \in[0, \pi]$ ), pois a instabilidade deste pedaço do ondulóide implica a instabilidade de todas as partes que encontram os planos $P_{1}$ e $P_{2}$ perpendicularmente ao longo de seu bordo. Tomamos 
a parametrização da curva ondulária $\gamma(t)=(x(t), 0, z(t))$, cuja rotação ao redor do eixo $\mathrm{Oz}$ gera este semiperíodo do ondulóide, como no caso (ii) do Teorema 4.4.1, onde

$$
\begin{aligned}
& x(t)=b\left(\frac{1-k \cos t}{1+k \cos t}\right)^{1 / 2} \\
& z(t)=\frac{b^{2}}{a} \int_{0}^{\pi} \frac{d \tau}{(1-k \cos \tau)^{1 / 2}(1+k \cos \tau)^{3 / 2}}
\end{aligned}
$$

$\operatorname{com} k \in[0,1[$.

Observamos que se $k=0$ então o ondulóide é um cilindro, visto que $a=b$, e já estudamos na seção 5.2 o critério de estabilidade para esta superfície.

Desta forma, nosso interesse é provar a instabilidade dos ondulóides para $k \in] 0,1[$. No entanto, veremos que, com o procedimento adotado, não será possível concluir este resultado de imediato, sendo necessário dividi-lo em 2 partes : $k \in] 0 ; 0,911[\mathrm{e}$ $k \in] 0,91 ; 1[$. Como ] $0 ; 0,911[\cap] 0,91 ; 1[\neq \emptyset$, então ficará provada a instabilidade para $k \in] 0,1[$.

\subsubsection{Instabilidade dos ondulóides para $k \in] 0 ; 0,911[$}

Teorema 5.4.1. Seja $S$ um semiperíodo do ondulóide, que encontra $P_{1}$ e $P_{2}$ perpendicularmente. A função $f(t)=\left(1-k^{2} \cos ^{2} t\right)^{3 / 4}(1+k \cos t)^{2} \cos t$ tem a propriedade do valor médio nulo

$$
\int_{S} f d a=2 \pi b^{2} \int_{0}^{\pi} f(t) \frac{(1-k \cos t)^{2}}{\left(1-k^{2} \cos ^{2} t\right)^{3 / 2}} d t=0
$$

e satisfaz a desigualdade

$$
\delta^{2} J=\int_{S}\left(\|\nabla f\|^{2}-\|B\|^{2} f^{2}\right) d a<0
$$

quando $k \in] 0 ; 0,911[$. Com isto, os ondulóides com $k \in] 0 ; 0,911[$ são instáveis.

Prova: Tomando a parametrização (5.34) da curva que gera a superfície rotacional S, calculamos a expressão $\delta^{2} J$ para uma variação rotacionalmente simétrica.

Observando que agora estamos considerando a curva na forma $\gamma(t)=(x(t), z(t))$ ao invés de $\gamma(t)=(x(t), t)$, obtemos que:

$$
\|\nabla f\|^{2}=\frac{1}{\dot{x}^{2}(t)+\dot{z}^{2}(t)} \dot{f}^{2}(t) .
$$


Mas, de acordo com os cálculos feitos na prova do caso (ii) do Teorema 4.4.1,temos que

$$
\|\nabla f\|^{2}=\frac{(1+k \cos t)^{2}}{b^{2}} \dot{f}^{2}(t) \text {. }
$$

Em geral, vale para a superfície rotacional S gerada por uma curva $\gamma(t)=(x(t), 0, z(t))$ que

$$
\|B\|^{2}=k_{1}^{2}+k_{2}^{2}=\frac{(\dot{x} \ddot{z}-\ddot{x} \dot{z})^{2}}{\left(\dot{x}^{2}+\dot{z}^{2}\right)^{3}}+\frac{\dot{z}^{2}}{x^{2}\left(\dot{x}^{2}+\dot{z}^{2}\right)}
$$

Mas, tomando a parametrização (5.34), bem como aproveitando os cálculos feitos em (ii) do Teorema 4.4.1, resulta que

$$
\begin{aligned}
& \ddot{x}(t)=b k \frac{\cos t\left(1-k^{2} \cos ^{2} t\right)+k \sin ^{2} t(1-2 k \cos t)}{(1+k \cos t)^{5 / 2}(1-k \cos t)^{3 / 2}} \\
& \text { e } \\
& \ddot{z}(t)=\frac{b^{2}}{a} k \sin t \frac{1-2 k \cos t}{(1+k \cos t)^{5 / 2}(1-k \cos t)^{3 / 2}} \\
& \text { Logo, }(\dot{x} \ddot{z}-\ddot{x} \dot{z})^{2}=\frac{b^{6}}{a^{2}} k^{2} \frac{\cos ^{2} t}{(1-k \cos t)^{2}(1+k \cos t)^{6}} \\
& \mathrm{e}\left(\dot{x}^{2}+\dot{z}^{2}\right)^{3}=\frac{b^{6}}{(1+k \cos t)^{6}} \text {. } \\
& \text { Então, } k_{1}^{2}=\frac{(\dot{x} \ddot{z}-\ddot{x} \dot{z})^{2}}{\left(\dot{x}^{2}+\dot{z}^{2}\right)^{3}}=\frac{k^{2}}{a^{2}} \frac{\cos ^{2} t}{(1-k \cos t)^{2}} \\
& k_{2}^{2}=\frac{\dot{z}^{2}}{x^{2}\left(\dot{x}^{2}+\dot{z}^{2}\right)}=\frac{1}{a^{2}} \frac{1}{(1-k \cos t)^{2}} \text {. }
\end{aligned}
$$

$$
\|B\|^{2}=k_{1}^{2}+k_{2}^{2}=\frac{1}{a^{2}} \frac{1+k^{2} \cos ^{2} t}{(1-k \cos t)^{2}} .
$$

Usando ainda a parametrização (5.34), temos que o elemento de área da superfície rotacional $\mathrm{S}$ é dado por

$$
d a=2 \pi x(t) \sqrt{\dot{x}^{2}(t)+\dot{z}^{2}(t)} d t=2 \pi b^{2} \frac{(1-k \cos t)^{1 / 2}}{(1+k \cos t)^{3 / 2}} d t .
$$

Juntando (5.35), (5.37) e (5.38), obtemos a expressão de $\delta^{2} J$ para o ondulóide: 


$$
\delta^{2} J=\frac{2 \pi b^{2}}{a^{2}} \int_{0}^{\pi}\left\{\frac{\left(1-k^{2} \cos ^{2} t\right)^{1 / 2}}{1-k^{2}} \dot{f}^{2}(t)-\frac{1+k^{2} \cos ^{2} t}{\left(1-k^{2} \cos ^{2} t\right)^{3 / 2}} f^{2}(t)\right\} d t
$$

Passemos agora a provar as afirmações deste teorema. Usando a expressão de $f(t)$ e (5.38) obtemos

$$
\int_{S} f(t) d a=2 \pi b^{2} \int_{0}^{\pi}\left(1-k^{2} \cos ^{2} t\right)^{5 / 4} \cos t d t
$$

Mas, observamos que a função $h(t):=p(t) q(t)$, onde $p(t):=\left(1-k^{2} \cos ^{2} t\right)^{5 / 4}$ e $q(t):=\cos t$, é ímpar com relação a $t=\pi / 2$, visto que $p(t)$ é par com relação a $t=\pi / 2$ e $q(t)$ é ímpar com relação a $t=\pi / 2$. Portanto,

$$
\int_{S} f(t) d a=2 \pi b^{2} \int_{0}^{\pi} h(t) d t=0 \text {, ou seja, a função } f(t) \text { dada tem a propriedade do }
$$
valor médio nulo.

Falta mostrar que para a função $\mathrm{f}$ vale $\delta^{2} J<0$, de onde seguirá a instabilidade do ondulóide. Por (5.39), $\delta^{2} J<0$ equivale a

$$
\int_{0}^{\pi}\left(1-k^{2} \cos ^{2} t\right)^{1 / 2} \dot{f}^{2}(t) d t<\left(1-k^{2}\right) \int_{0}^{\pi} \frac{1+k^{2} \cos ^{2} t}{\left(1-k^{2} \cos ^{2} t\right)^{3 / 2}} f^{2}(t) d t
$$

Com a expressão de $f(t)$, calculamos:

$$
\begin{aligned}
f^{2}(t) & =\left(1-k^{2} \cos ^{2} t\right)^{3 / 2}(1+k \cos t)^{4} \cos ^{2} t \\
\dot{f}(t) & =\sin t(1+k \cos t)\left\{\frac{3}{2} \frac{1+k \cos ^{2} t}{\left(1-k^{2} \cos ^{2} t\right)^{1 / 4}} k^{2} \cos ^{2} t-2\left(1-k^{2} \cos ^{2} t\right)^{3 / 4} k \cos t-\right. \\
& \left.-\left(1-k^{2} \cos ^{2} t\right)^{3 / 4}(1+k \cos t)\right\}
\end{aligned}
$$

e

$$
\begin{aligned}
& \dot{f}^{2}(t)=\sin ^{2} t(1+k \cos t)^{2}\left\{\frac{9}{4} \frac{(1+k \cos t)^{2}}{\left(1-k^{2} \cos ^{2} t\right)^{1 / 2}} k^{4} \cos ^{4} t+4\left(1-k^{2} \cos ^{2} t\right)^{3 / 2} k^{2} \cos ^{2} t+\right. \\
& +\left(1-k^{2} \cos ^{2} t\right)^{3 / 2}(1+k \cos t)^{2}-6\left(1-k^{2} \cos ^{2} t\right)^{1 / 2}(1+k \cos t) k^{3} \cos ^{3} t- \\
& \left.-3\left(1-k^{2} \cos ^{2} t\right)^{1 / 2}(1+k \cos t)^{2} k^{2} \cos ^{2} t+4\left(1-k^{2} \cos ^{2} t\right)^{3 / 2}(1+k \cos t) k \cos t\right\} .
\end{aligned}
$$

Portanto, o lado esquerdo de (5.40) fica dado por 


$$
\begin{aligned}
& \int_{0}^{\pi}\left(1-k^{2} \cos ^{2} t\right)^{1 / 2} \dot{f}^{2}(t) d t= \\
& =\int_{0}^{\pi} \sin ^{2} t\left(1-k^{2} \cos ^{2} t\right)^{2}(1+k \cos t)^{2}\left\{4 k^{2} \cos ^{2} t+(1+k \cos t)^{2}+\right. \\
& +4(1+k \cos t) k \cos t\} d t- \\
& -\int_{0}^{\pi} \sin ^{2} t\left(1-k^{2} \cos ^{2} t\right)(1+k \cos t)^{3}\left\{6 k^{3} \cos ^{3} t+3(1+k \cos t) k^{2} \cos ^{2} t\right\} d t+ \\
& \left.+\frac{9}{4} \int_{0}^{\pi} \sin ^{2} t(1+k \cos t)^{4} k^{4} \cos ^{4} t\right\} d t:=I_{1}-I_{2}+I_{3} .
\end{aligned}
$$

Mas, calculando as integrais acima $I_{1}, I_{2}$ e $I_{3}$, resulta que :

$$
\begin{aligned}
& I_{1}=\pi\left\{\frac{1}{2}+\frac{20}{8} k^{2}-\frac{34}{16} k^{4}+\frac{20}{128} k^{6}+\frac{63}{256} k^{8}\right\}, \\
& I_{2}=\pi\left\{\frac{3}{8} k^{2}+\frac{33}{16} k^{4}-\frac{135}{128} k^{6}-\frac{63}{256} k^{8}\right\} \\
& I_{3}=\frac{9}{4} \pi\left\{\frac{1}{16} k^{4}+\frac{30}{128} k^{6}+\frac{7}{256} k^{8}\right\}
\end{aligned}
$$

de onde segue que o lado esquerdo de (5.40) é igual a

$$
\int_{0}^{\pi}\left(1-k^{2} \cos ^{2} t\right)^{1 / 2} \dot{f}^{2}(t) d t=\pi\left\{\frac{1}{2}+\frac{17}{8} k^{2}-\frac{259}{64} k^{4}+\frac{445}{256} k^{6}+\frac{567}{1024} k^{8}\right\} .
$$

Para o lado direito de (5.40), calculamos:

$$
\begin{aligned}
\left(1-k^{2}\right) \int_{0}^{\pi} \frac{1+k^{2} \cos ^{2} t}{\left(1-k^{2} \cos ^{2} t\right)^{3 / 2}} f^{2}(t) d t & = \\
& =\pi\left\{\frac{1}{2}+\frac{17}{8} k^{2}-\frac{7}{16} k^{4}-\frac{245}{128} k^{6}-\frac{35}{128} k^{8}\right\} .
\end{aligned}
$$

Juntando (5.40), (5.41) e (5.42) concluímos que mostrar $\delta^{2} J<0$ para a função f escolhida, equivale a mostrar que

$$
-\frac{259}{64} k^{4}+\frac{445}{256} k^{6}+\frac{567}{1024} k^{8}<-\frac{7}{16} k^{4}-\frac{245}{128} k^{6}-\frac{35}{128} k^{8}
$$

ou seja,

$$
k^{4}\left(-3696+3740 k^{2}+847 k^{4}\right)<0 .
$$

No entanto, a desigualdade (5.43) é satisfeita para $k \in] 0 ; 0,911$ ] e portanto, mostramos a instabilidade dos ondulóides neste intervalo. 


\subsubsection{Instabilidade dos ondulóides para $k \in[0,91 ; 1[$}

Com o que foi mostrado na subseção anterior, não foi possível mostrar, no entanto, que os ondulóides com $k \in[0,91 ; 1$ [ são instáveis, pois a desigualdade (5.43) não é válida para todos os valores neste intervalo. A seguir, vamos tentar reparametrizar a função $f(t)$ dada no Teorema 5.4.1 de modo a provar a instabilidade no caso $k \in[0,91 ; 1$ [, que é o resultado principal desta subseção. Tendo em vista a tentativa de obtermos tal reparametrização de $f(t)$, podemos transpor o problema

" Procuramos f para a qual vale

$$
\delta^{2} \tilde{J}:=\frac{a^{2}\left(1-k^{2}\right)}{2 \pi b^{2}} \delta^{2} J=\int_{0}^{\pi}\left(a(t) \dot{f}^{2}(t)-b(t) f^{2}(t)\right) d t<0,
$$

sob a condição adicional

$$
\int_{0}^{\pi} p(t) f(t) d t=0
$$

$\operatorname{com} a(t):=\left(1-k^{2} \cos ^{2} t\right)^{1 / 2}, \quad b(t):=\left(1-k^{2}\right) \frac{1+k^{2} \cos ^{2} t}{\left(1-k^{2} \cos ^{2} t\right)^{3 / 2}}$ e $p(t):=\frac{(1-k \cos t)^{2}}{\left(1-k^{2} \cos ^{2} t\right)^{3 / 2}} . "$ para a forma

"Procuramos g para a qual vale

$$
\delta^{2} \tilde{J}:=\int_{0}^{\pi}\left(g^{\prime 2}(s)-c(s) g^{2}(s)\right) d s<0,
$$

sob a condição adicional

$$
\int_{0}^{\pi} g(s) d s=0
$$

onde " " " denota a derivação em relação a s."

Consideramos a seguinte mudança de parâmetros.

$$
\left\{\begin{array}{cl}
s & =\phi(t) \\
f(t) & =h(t) g(s) \\
\phi(0) & =0, \quad \phi(\pi)=\pi
\end{array}\right.
$$

Nosso objetivo é obter uma expressão para $\phi(t)$, de modo que (5.46) e (5.47) sejam satisfeitas.

Por (5.48), temos que 
$\dot{f}(t)=\dot{h}(t) g(s)+h(t) g^{\prime}(s) \dot{\phi}(t)$, onde $g^{\prime}(s)=\frac{d}{d s} g(s)$ e $\quad \dot{\phi}(t)=\frac{d s}{d t}$.

Logo,

$$
\dot{f}^{2}(t)=\dot{h}^{2}(t) g^{2}(s)+h^{2}(t) g^{\prime 2}(s) \dot{\phi}^{2}(t)+2 h(t) \dot{h}(t) g(s) g^{\prime}(s) \dot{\phi}(t) .
$$

Visto que $s=\phi(t)$ é uma mudança de parâmetros, então $\dot{\phi}(t) \neq 0, \quad \forall t \in[0, \pi]$ e temos que $\frac{d t}{d s}=\frac{1}{\dot{\phi}(t)}$.

Portanto, com a mudança (5.48), a expressão (5.44) fica dada por

$$
\begin{aligned}
\delta^{2} \tilde{J} & =\int_{0}^{\pi}\left\{a(t)\left[\dot{h}^{2}(t) g^{2}(s)+h^{2}(t) g^{\prime 2}(s) \dot{\phi}^{2}(t)+2 h(t) \dot{h}(t) g(s) g^{\prime}(s) \dot{\phi}(t)\right]-\right. \\
& \left.-b(t) h^{2}(t) g^{2}(s)\right\} \frac{1}{\dot{\phi}(t)} d s .
\end{aligned}
$$

Como queremos que o coeficiente de $g^{\prime 2}(s)$ em (5.46) seja igual a 1 , então segue de (5.49) que:

$$
a(t) h^{2}(t) \dot{\phi}(t)=1,
$$

ou seja, $h(t) \neq 0, \quad \forall t \in[0, \pi]$.

Devido a (5.45), (5.48) e (5.47), temos ainda que deve valer

$$
p(t) h(t) \frac{1}{\dot{\phi}(t)}=1 .
$$

Substituindo em (5.50) e (5.51) as expressões de $a(t)$ e $p(t)$, obtemos o sistema

$$
\left\{\begin{array}{l}
\left(1-k^{2} \cos ^{2} t\right)^{1 / 2} h^{2}(t) \dot{\phi}(t)=1 \\
\frac{(1-k \cos t)^{2}}{\left(1-k^{2} \cos ^{2} t\right)^{3 / 2}} h(t)=\dot{\phi}(t) \\
\phi(0)=0, \quad \phi(\pi)=\pi
\end{array}\right.
$$

Resolvendo o sistema (5.52), segue que

$$
h(t)=\left(\frac{1+k \cos t}{1-k \cos t}\right)^{1 / 3} \quad \text { e } \dot{\phi}(t)=\frac{(1-k \cos t)^{1 / 6}}{(1+k \cos t)^{7 / 6}}, \quad \text { ou seja, }
$$

$\phi(t)=c \int_{0}^{t} \frac{(1-k \cos \tau)^{1 / 6}}{(1+k \cos \tau)^{7 / 6}} d \tau+d$, onde c e d são constantes de integração. 
Observando que devem valer $\phi(0)=0$ e $\phi(\pi)=\pi$, então

$$
h(t)=\left(\frac{1+k \cos t}{1-k \cos t}\right)^{1 / 3} \quad \text { e } \quad \phi(t)=\pi \frac{\int_{0}^{t} \frac{(1-k \cos \tau)^{1 / 6}}{(1+k \cos \tau)^{7 / 6}} d \tau}{\int_{0}^{\pi} \frac{(1-k \cos \tau)^{1 / 6}}{(1+k \cos \tau)^{7 / 6}} d \tau}
$$

Notamos que devido à expressão de $\phi(t)$ acima, o fator para que $g^{\prime 2}(s)$ não é mais 1 , mas ainda continua sendo uma constante.

A fim de procedermos como no Teorema 5.4.1, tomamos $f(t) \operatorname{como} f(t)=h(t) \cos (\phi(t))$, onde $h(t)$ e $\phi(t)$ estão dados em (5.53). Temos então o seguinte resultado, que prova a instabilidade dos ondulóides com $k \in[0,91 ; 1[$.

Teorema 5.4.2. Seja $S$ um semiperiodo do ondulóide, que encontra $P_{1}$ e $P_{2}$ perpendicularmente. A função $f(t)=h(t) g(s)=h(t) \cos (\phi(t))$, com $h(t)$ e $\phi(t)$ dadas por (5.53), tem valor médio nulo $\int_{S} f(t) d a=0$ e satisfaz a desigualdade $\delta^{2} J<0$, para ondulóides com $k \in[0,91 ; 1[$.

Prova: Começamos mostrando que $f(t)$ tem a propriedade do valor médio nulo, pois temos por (5.51) que

$$
\int_{S} f(t) d a=2 \pi b^{2} \int_{0}^{\pi} h(t) \cos (\phi(t)) \frac{(1-k \cos t)^{2}}{\left(1-k^{2} \cos ^{2} t\right)^{3 / 2}} \frac{1}{\dot{\phi}(t)} d s=2 \pi b^{2} \int_{0}^{\pi} \cos s d s=0 .
$$

Calculamos agora a expressão de $\delta^{2} J$ para a função $f(t)$ escolhida, com base em (5.44). Como $f(t)=h(t) g(s)$, então por (5.53) resulta que:

$$
\dot{f}(t)=-\frac{2}{3} \frac{k \sin t}{(1+k \cos t)^{2 / 3}(1-k \cos t)^{4 / 3}} g(\phi(t))+\frac{(1+k \cos t)^{1 / 3}}{(1-k \cos t)^{1 / 3}} g^{\prime}(s) \dot{\phi}(t)
$$

e

$$
\begin{aligned}
\dot{f}^{2}(t) & =\frac{4}{9} \frac{k^{2} \sin ^{2} t g^{2}(\phi(t))}{(1+k \cos t)^{4 / 3}(1-k \cos t)^{8 / 3}}+\frac{(1+k \cos t)^{2 / 3}}{(1-k \cos t)^{2 / 3}} g^{\prime 2}(s) \dot{\phi}^{2}(t)- \\
& -\frac{4}{3} \frac{k \sin t}{(1+k \cos t)^{1 / 3}(1-k \cos t)^{5 / 3}} g(\phi(t)) g^{\prime}(s) \dot{\phi}(t) .
\end{aligned}
$$

Portanto, temos por (5.44) que 


$$
\begin{aligned}
\delta^{2} \tilde{J} & :=\frac{\left(1-k^{2}\right) a^{2}}{2 \pi b^{2}} \delta^{2} J=\int_{0}^{\pi} \frac{4}{9} \frac{k^{2} \sin ^{2} t g^{2}(s)}{(1+k \cos t)^{5 / 6}(1-k \cos t)^{13 / 6}} d t+ \\
& +\int_{0}^{\pi} \frac{(1+k \cos t)^{7 / 6}}{(1-k \cos t)^{1 / 6}} g^{\prime 2}(s) \dot{\phi}^{2}(t) d t- \\
& -\int_{0}^{\pi} \frac{4}{3} \frac{k \sin t(1+k \cos t)^{1 / 6}}{(1-k \cos t)^{7 / 6}} g(s) g^{\prime}(s) \dot{\phi}(t) d t- \\
& -\left(1-k^{2}\right) \int_{0}^{\pi} \frac{\left(1+k^{2} \cos ^{2} t\right)}{(1-k \cos t)^{13 / 6}(1+k \cos t)^{5 / 6}} g^{2}(s) d t= \\
& :=I_{1}+I_{2}+I_{3}+I_{4} .
\end{aligned}
$$

Mas, integrando $I_{3}$ por partes e usando que $g(s)=\cos s$, resulta que

$$
I_{3}=\frac{2}{3} \int_{0}^{\pi} g^{2}(s)\left\{\frac{k \cos t(1+k \cos t)^{1 / 6}}{(1-k \cos t)^{7 / 6}}-\frac{k^{2} \sin ^{2} t}{(1+k \cos t)^{5 / 6}(1-k \cos t)^{13 / 6}}\left[\frac{4}{3}+k \cos t\right]\right\} d t .
$$

Portanto, temos que

$$
\begin{aligned}
I_{1}+I_{3}+I_{4} & =\int_{0}^{\pi} \frac{g^{2}(s)}{(1-k \cos t)^{13 / 6}(1+k \cos t)^{5 / 6}}\left\{\frac{2}{3} k \cos t\left(1-k^{2} \cos ^{2} t\right)-\right. \\
& \left.-k^{2} \sin ^{2} t\left(\frac{4}{9}+\frac{2}{3} k \cos t\right)-\left(1-k^{2}\right)\left(1+k^{2} \cos ^{2} t\right)\right\} d t .
\end{aligned}
$$

No, entanto, trocando $\sin ^{2} t$ por $1-\cos ^{2} t$ na igualdade acima, vemos que

$$
\begin{aligned}
& \frac{2}{3} k \cos t\left(1-k^{2} \cos ^{2} t\right)-k^{2} \sin ^{2} t\left(\frac{4}{9}+\frac{2}{3} k \cos t\right)-\left(1-k^{2}\right)\left(1+k^{2} \cos ^{2} t\right)= \\
& =-\left(1-k^{2}\right)\left(1+k^{2} \cos ^{2} t-\frac{2}{3} k \cos t\right)-\frac{4}{9} k^{2}\left(1-\cos ^{2} t\right)+\frac{4}{9} k^{4} \cos ^{2} t- \\
& -\frac{4}{9} k^{4} \cos ^{2} t= \\
& =-\left(1-k^{2}\right)\left(1+\frac{5}{9} k^{2} \cos ^{2} t-\frac{2}{3} k \cos t\right)-\frac{4}{9} k^{2}\left(1-k^{2} \cos ^{2} t\right) .
\end{aligned}
$$

Notamos que, para $k \in[0,1$ [, vale que $1-k \cos t$ e $1+k \cos t$ são positivos. Além disto,

$$
1+\frac{5}{9} k^{2} \cos ^{2} t-\frac{2}{3} k \cos t=\frac{5}{9} k^{2} \cos ^{2} t+\frac{1}{3}+\frac{2}{3}(1-k \cos t)>0 .
$$

Consequentemente, (5.56) é constituída pela soma de parcelas negativas, o que implica que a soma $I_{1}+I_{3}+I_{4}$, dada por (5.55) é negativa. 
Para o cálculo de $I_{2}$ usamos (5.48) e a expressão de $\phi(t)$ dada em (5.53)

$$
\int_{0}^{\pi} \frac{(1+k \cos t)^{7 / 6}}{(1-k \cos t)^{1 / 6}} g^{\prime 2}(s) \dot{\phi}^{2}(t) d t=\int_{0}^{\pi}\left\{\frac{\pi}{\int_{0}^{\pi} \frac{(1-k \cos t)^{1 / 6}}{(1+k \cos t)^{7 / 6}}}\right\} g^{\prime 2}(s) d s
$$

Portanto, (5.54) fica:

$$
\begin{aligned}
& \frac{\left(1-k^{2}\right) a^{2}}{2 \pi b^{2}} \delta^{2} J=\int_{0}^{\pi}\left\{\frac{\pi}{\int_{0}^{\pi} \frac{(1-k \cos t)^{1 / 6}}{(1+k \cos t)^{7 / 6}}}\right\} g^{\prime 2}(s) d s+ \\
& +\int_{0}^{\pi} \frac{g^{2}(s)}{(1-k \cos t)^{13 / 6}(1+k \cos t)^{5 / 6}}\left\{-\left(1-k^{2}\right)\left(1+\frac{5}{9} k^{2} \cos ^{2} t-\frac{2}{3} k \cos t\right)-\right. \\
& \left.-\frac{4}{9} k^{2}\left(1-k^{2} \cos ^{2} t\right)\right\} d t .
\end{aligned}
$$

Sabendo que $g(s)=\cos s$, temos ainda que:

$$
\int_{0}^{\pi}\left\{\frac{\pi}{\int_{0}^{\pi} \frac{(1-k \cos t)^{1 / 6}}{(1+k \cos t)^{7 / 6}} d t}\right\} g^{\prime 2}(s) d s=\frac{\pi^{2}}{2 \int_{0}^{\pi} \frac{(1-k \cos t)^{1 / 6}}{(1+k \cos t)^{7 / 6}} d t}
$$

Desta forma, mostrar que $\delta^{2} J<0$, para a função $f(t)$ escolhida, equivale a mostrar que

$$
\begin{aligned}
\frac{\pi^{2}}{2 \int_{0}^{\pi} \frac{(1-k \cos t)^{1 / 6}}{(1+k \cos t)^{7 / 6}} d t}<\int_{0}^{\pi} \frac{\cos ^{2} s}{(1-k \cos t)^{13 / 6}(1+k \cos t)^{5 / 6}}\left\{\left(1-k^{2}\right)\right. \\
\left.\left(1+\frac{5}{9} k^{2} \cos ^{2} t-\frac{2}{3} k \cos t\right)+\frac{4}{9} k^{2}\left(1-k^{2} \cos ^{2} t\right)\right\} d t .
\end{aligned}
$$

Como para $k<1$, o termo $\left(1-k^{2}\right)\left(1+\frac{5}{9} k^{2} \cos ^{2} t-\frac{2}{3} k \cos t\right)$ é positivo, então basta mostrar que

$$
\begin{aligned}
& \frac{\pi^{2}}{2 \int_{0}^{\pi} \frac{(1-k \cos t)^{1 / 6}}{(1+k \cos t)^{7 / 6}} d t}<\frac{4}{9} k^{2} \int_{0}^{\pi} \frac{\cos ^{2} s(1+k \cos t)^{1 / 6}}{(1-k \cos t)^{7 / 6}} d t, \text { ou seja, } \\
& \pi^{2}<\frac{8}{9} k^{2} \int_{0}^{\pi} \frac{\cos ^{2} s(1+k \cos t)^{1 / 6}}{(1-k \cos t)^{7 / 6}} d t \int_{0}^{\pi} \frac{(1-k \cos t)^{1 / 6}}{(1+k \cos t)^{7 / 6}} d t
\end{aligned}
$$

de onde seguirá $\delta^{2} J<0$. Provamos (5.57) com os itens (i) e (ii) a seguir.

Para $t=0$ e portanto, $s=0$, temos que o lado direito de (5.57) é igual a $\frac{8}{9} \pi^{2} \frac{k^{2}}{1-k^{2}}$. 
No entanto, para $k \in\left[0,91 ; 1\right.$ [ temos que $\frac{8}{9} \pi^{2} \frac{k^{2}}{1-k^{2}}>\pi^{2}$ e, portanto, (5.57) vale para $t=0$.

(i) Supomos que $s=\phi(t) \leq \pi / 4, \forall t \in] 0, \pi / 2$ ] ( provaremos este fato no item (ii)). Logo, $\left.\left.g^{2}(s)=\cos ^{2} s \geq 1 / 2, \forall t \in\right] 0, \pi / 2\right]$ e vale que

$$
\begin{aligned}
\int_{0}^{\pi} \frac{(1+k \cos t)^{1 / 6}}{(1-k \cos t)^{7 / 6}} \cos ^{2} s d t & =\int_{0}^{\pi / 2} \frac{(1+k \cos t)^{1 / 6}}{(1-k \cos t)^{7 / 6}} \cos ^{2} s d t+\int_{\pi / 2}^{\pi} \frac{(1+k \cos t)^{1 / 6}}{(1-k \cos t)^{7 / 6} \cos ^{2} s d t} \\
& \geq \frac{1}{2} \int_{0}^{\pi / 2} \frac{(1+k \cos t)^{1 / 6}}{(1-k \cos t)^{7 / 6}} d t
\end{aligned}
$$

Além disto, chamando $I:=\int_{0}^{\pi / 2} \frac{(1+k \cos t)^{1 / 6}}{(1-k \cos t)^{7 / 6}} d t$, e tomando

$$
\tilde{g}(t):=(1+k \cos t)^{1 / 6} \text {, então } I=\int_{0}^{\pi / 2} \frac{\tilde{g}(t)}{(1-k \cos t)^{7 / 6}} d t \text {. Considerando a mudança }
$$

de parâmetros $\tau=k \cos t$ e definindo $\hat{g}(\tau):=\frac{\tilde{g}(t)}{\sqrt{k+\tau}}$, resulta que

$I=\int_{0}^{k} \frac{\hat{g}(\tau)}{(1-\tau)^{7 / 6} \sqrt{k-\tau}} d \tau$. Fazendo outra mudança de parâmetros $z=1-\tau$ e $\lambda:=1-k$, temos que $I=\int_{\lambda}^{1} \frac{\hat{g}(\tau)}{z^{10 / 6} \sqrt{1-\lambda / z}} d z$. E finalmente com a mudança de parâmetros $u=\frac{\lambda}{z}$ obtemos $I=\int_{\lambda}^{1} \frac{\hat{g}(\tau)}{\lambda^{2 / 3} u^{1 / 3} \sqrt{1-u}} d u$.

Como $\delta:=\sqrt{\lambda}>\lambda$, visto que $\lambda \in] 0,1[$, separamos I como

$$
I=\int_{\lambda}^{\delta} \frac{\hat{g}(\tau)}{\lambda^{2 / 3} u^{1 / 3} \sqrt{1-u}} d u+\int_{\delta}^{1} \frac{\hat{g}(\tau)}{\lambda^{2 / 3} u^{1 / 3} \sqrt{1-u}} d u:=I_{1}+I_{2} .
$$

No entanto, observamos que

$$
\hat{g}(\tau)=\frac{\hat{g}(t)}{\sqrt{k+\tau}}=\frac{(1+k \cos t)^{1 / 6}}{(k+k \cos t)^{1 / 2}} \geq \frac{1}{(k+k \cos t)^{1 / 2}} \geq \frac{1}{\sqrt{2 k}},
$$

pois $t \in] 0, \pi / 2]$, o que implica $1+k \cos t \geq 1$ e $k+k \cos t \leq 2 k$.

Em $I_{1}$, temos que $u \in[\lambda, \delta]$, isto é, $1-u \leq 1-\lambda=k$ e $\sqrt{1-u} \leq \sqrt{k}$, ou seja,em $I_{1}$ temos que

$$
\frac{\hat{g}(\tau)}{\sqrt{1-u}} \geq \frac{1}{\sqrt{1-u}} \frac{1}{\sqrt{2 k}} \geq \frac{1}{\sqrt{k}} \frac{1}{\sqrt{2 k}}=\frac{1}{\sqrt{2} k} .
$$


Em $I_{2}$, temos que $u \in[\delta, 1] \mathrm{e}$, com isto, $u \leq 1$. Portanto, temos em $I_{2}$ que

$$
\frac{\hat{g}(\tau)}{u^{1 / 3}} \geq \frac{1}{\sqrt{2 k}} \frac{1}{u^{1 / 3}} \geq \frac{1}{\sqrt{2 k}} .
$$

Assim:

$$
\begin{aligned}
I_{1} & \geq \frac{1}{\sqrt{2} k \lambda^{2 / 3}} \int_{\lambda}^{\delta} \frac{d u}{u^{1 / 3}}=\frac{3}{2} \frac{1}{\sqrt{2} k \lambda^{2 / 3}}\left(\delta^{2 / 3}-\lambda^{2 / 3}\right)=\frac{3}{2} \frac{1}{\sqrt{2} k}\left(\left(\frac{\delta}{\lambda}\right)^{2 / 3}-1\right)= \\
& =\frac{3}{2} \frac{1}{\sqrt{2} k}\left(\left(\frac{1}{\sqrt{\lambda}}\right)^{2 / 3}-1\right)=\frac{3}{2} \frac{1}{\sqrt{2} k}\left(\left(\frac{1}{\sqrt{1-k}}\right)^{2 / 3}-1\right)
\end{aligned}
$$

e

$I_{2} \geq \frac{1}{\sqrt{2 k} \lambda^{2 / 3}} \int_{\delta}^{1} \frac{d u}{\sqrt{1-u}}=\frac{2}{\sqrt{2 k} \lambda^{2 / 3}}(\sqrt{1-\delta})=\frac{2}{\sqrt{2 k}(1-k)^{2 / 3}}(1-\sqrt{1-k})^{1 / 2}$.

Observando que $\int_{\pi / 2}^{\pi} \frac{(1-k \cos t)^{1 / 6}}{(1+k \cos t)^{7 / 6}} d t=\int_{0}^{\pi / 2} \frac{(1+k \cos t)^{1 / 6}}{(1-k \cos t)^{7 / 6}} d t=I$ e portanto,

$$
\int_{0}^{\pi} \frac{(1-k \cos t)^{1 / 6}}{(1+k \cos t)^{7 / 6}} d t=\int_{0}^{\pi / 2} \frac{(1-k \cos t)^{1 / 6}}{(1+k \cos t)^{7 / 6}} d t+\int_{\pi / 2}^{\pi} \frac{(1-k \cos t)^{1 / 6}}{(1+k \cos t)^{7 / 6}} d t \geq I, \text { segue }
$$

que o lado direito de (5.57) é tal que:

$$
\begin{aligned}
& \frac{8}{9} k^{2} \int_{0}^{\pi} \frac{\cos ^{2} s(1+k \cos t)^{1 / 6}}{(1-k \cos t)^{7 / 6}} d t \int_{0}^{\pi} \frac{(1-k \cos t)^{1 / 6}}{(1+k \cos t)^{7 / 6}} d t \geq \\
& \geq \frac{8}{9} k^{2} \frac{1}{2} I^{2}=\frac{4}{9} k^{2}\left(I_{1}+I_{2}\right)^{2} \geq \\
& \geq \frac{4}{9} k^{2}\left\{\frac{3}{2} \frac{1}{\sqrt{2} k}\left(\left(\frac{1}{\sqrt{1-k}}\right)^{2 / 3}-1\right)+\frac{2}{\sqrt{2 k}(1-k)^{2 / 3}}(1-\sqrt{1-k})^{1 / 2}\right\}^{2} \geq \\
& \geq \frac{4}{9} k^{2} \frac{4}{2 k(1-k)^{4 / 3}}(1-\sqrt{1-k})=\frac{8}{9} \frac{k(1-\sqrt{1-k})}{(1-k)^{4 / 3}} \geq \\
& \geq \frac{8}{9} \frac{0,91(1-\sqrt{1-0,91})}{(1-0,91)^{4 / 3}} \cong 13,99>\pi^{2},
\end{aligned}
$$

visto que $k \in\left[0,91 ; 1\left[\right.\right.$. Portanto (5.57) fo provada, de onde segue que $\delta^{2} J<0$ para a função f escolhida.

(ii) Provamos agora que $s=\phi(t) \leq \pi / 4, \forall t \in] 0, \pi / 2]$.

Pela expressão de $\phi(t)$, dada em (5.53), isto equivale a mostrar que

$4 \int_{0}^{\pi / 2} \frac{(1-k \cos t)^{1 / 6}}{(1+k \cos t)^{7 / 6}} d t \leq \int_{0}^{\pi} \frac{(1-k \cos t)^{1 / 6}}{(1+k \cos t)^{7 / 6}} d t$, ou seja

$$
3 \int_{0}^{\pi / 2} \frac{(1-k \cos t)^{1 / 6}}{(1+k \cos t)^{7 / 6}} d t \leq \int_{\pi / 2}^{\pi} \frac{(1-k \cos t)^{1 / 6}}{(1+k \cos t)^{7 / 6}} d t .
$$


Notamos que, como $t \in] 0, \pi / 2$ ] e $k>0$, então $1-k \cos t \leq 1$ e $1+k \cos t \geq 1$.

Logo, $\frac{(1-k \cos t)^{1 / 6}}{(1+k \cos t)^{7 / 6}} \leq 1$ e, por (5.58) temos que basta mostrar

$$
\frac{3 \pi}{2} \leq \int_{\pi / 2}^{\pi} \frac{(1-k \cos t)^{1 / 6}}{(1+k \cos t)^{7 / 6}} d t=I .
$$

Mas, mostramos em (i) que

$$
\begin{aligned}
I & \geq \frac{3}{2} \frac{1}{\sqrt{2} k}\left(\left(\frac{1}{\sqrt{1-k}}\right)^{2 / 3}-1\right)+\frac{2}{\sqrt{2 k}(1-k)^{2 / 3}}(1-\sqrt{1-k})^{1 / 2} \geq \\
& \geq \frac{3}{2} \frac{1}{\sqrt{2} \cdot 0,91}\left(\left(\frac{1}{\sqrt{1-0,91}}\right)^{2 / 3}-1\right)+\frac{2}{\sqrt{2 \cdot 0,91}(1-0,91)^{2 / 3}}(1-\sqrt{1-0,91})^{1 / 2} \geq \\
& >\frac{3}{2} \pi
\end{aligned}
$$

pois $k \in[0,91 ; 1$ [. Mas, isto prova $(5.59)$ e, portanto, (ii) está concluída.

Conclusão: Até aqui, mostramos que os mínimos relativos do nosso problema variacional (P) são uma semiesfera de raio $\mathrm{r}, r<d$, onde $d:=\operatorname{dist}\left(P_{1}, P_{2}\right)$, e um cilindro de raio $\rho$, com $\rho \pi \geq d$, que estão localizados entre os planos $P_{1}$ e $P_{2}$ e com bordo contido neste planos. 



\section{Observações finais}

Neste capítulo, comparamos as áreas de um cilindro e de uma semiesfera, com mesmo volume, a fim de reconhecer um mínimo absoluto para o problema variacional (P). Em seguida, mostramos que a superfície S, cuja área assume um mínimo absoluto, deve ser conexa.

\subsection{Comparação das áreas de uma semiesfera e de um cilindro}

Consideramos uma semiesfera de raio $\mathrm{r}, r<d, d:=\operatorname{dist}\left(P_{1}, P_{2}\right)$ e um cilindro de raio $\rho$, que estão localizados entre $P_{1}$ e $P_{2}$ e com bordo contido nestes planos. Determinamos, então, para qual relação entre o volume $\mathrm{V}$ encerrado por estas superfícies e a distância d entre os planos $P_{1}$ e $P_{2}$, obtemos o mínimo absoluto de (P).

Começamos observando que se vale $\rho \pi<d$, então o cilindro é instável. Como a semiesfera de raio $\mathrm{r}$ é sempre estável, então no caso $\rho \pi<d$ a semiesfera é o mínimo absoluto de $(\mathrm{P})$, visto que o cilindro é excluído como solução.

Passamos agora a comparar a área do cilindro com a da semiesfera, supondo ainda que elas encerram o mesmo volume $\mathrm{V}$.

Para o cilindro de raio $\rho$, temos que

$$
A(\rho)=2 \pi \rho d, \quad V(\rho)=\pi \rho^{2} d
$$


e para a semiesfera de raio $r$, temos que

$$
A(r)=2 \pi r^{2}, \quad V(r)=\frac{2}{3} \pi r^{3} .
$$

Como supomos $V(\rho)=V(r)=V$, então deve valer que

$$
\pi \rho^{2} d=\frac{2}{3} \pi r^{3}, \quad \text { ou seja, } \rho=\left(\frac{2}{3} \frac{r^{3}}{d}\right)^{1 / 2} \text {. }
$$

Desta forma, $A(\rho) \geq A(r)$ se e somente se $2 \pi \rho d \geq 2 \pi r^{2}$, isto é,

$$
\rho d \geq r^{2}
$$

Por (6.1), temos que

$$
A(\rho) \geq A(r) \Longleftrightarrow\left(\frac{2}{3} \frac{r^{3}}{d}\right)^{1 / 2} d \geq r^{2} .
$$

Elevando ao quadrado a desigualdade do lado direito de (6.3), resulta que

$$
A(\rho) \geq A(r) \quad \Longleftrightarrow \quad \frac{2}{3} d \geq r
$$

Multiplicando (6.2) por $\mathrm{r}$, temos que $r \rho d \geq r^{3}$. Mas, por (6.1) temos que $r^{3}=\frac{3}{2} \rho^{2} d$ e, portanto, $r \rho d \geq r^{3}$ implica que $r \geq \frac{3}{2} \rho$. Elevando ao cubo, temos $r^{3} \geq \frac{3}{2}\left(\frac{3}{2}\right)^{2} \rho^{3}$ e usando novamente $r^{3}=\frac{3}{2} \rho^{2} d$, resulta então que $d \geq\left(\frac{3}{2}\right)^{2} \rho$, ou seja,

$$
\rho \leq\left(\frac{2}{3}\right)^{2} d=\frac{4}{9} d
$$

Portanto, para o cilindro temos que,

$$
A(\rho) \geq A(r) \Longleftrightarrow \rho \leq\left(\frac{2}{3}\right)^{2} d .
$$

Concluímos então que se vale a relação $\rho \leq\left(\frac{2}{3}\right)^{2} d$, a semiesfera de raio $\mathrm{r}$ tem área menor que o cilindro de raio $\rho$ que encerra o mesmo volume que ela e portanto, a semiesfera é , neste caso, o mínimo absoluto do problema variacional $(\mathrm{P})$.

Finalmente, observamos por (6.4) que se vale a relação $\frac{2}{3} d \leq r<d$, então o cilindro é o mínimo absoluto para (P). Por (6.6) isto também significa que $\rho \geq\left(\frac{2}{3}\right)^{2} d$. 
Logo $\pi \rho \geq \pi\left(\frac{2}{3}\right)^{2} d=\frac{4 \pi}{9} d>d$, ou seja, estamos na região de estabilidade do cilindro.

\subsection{Os mínimos absolutos de (P) são superfícies co- nexas}

No capítulo anterior, mostramos que os mínimos relativos do problema (P) são uma semiesfera de raio $\mathrm{r}, r<d$, ou um cilindro de raio $\rho$ com $\pi \rho \geq d$. No entanto, estas soluções foram assim obtidas, pois supomos no Teorema 3.2.1 que a superfície $\mathrm{S}$ fosse conexa.

Analisamos agora a possibilidade de S ter várias componentes conexas, formadas pelas combinações de cilindro e semiesfera, que são os mínimos relativos de $(\mathrm{P})$. Queremos, portanto, procurar por mínimos absolutos de $(\mathrm{P})$.

Vamos proceder por indução no número de componentes conexas de S. Primeiramente, tratamos das possibilidades de duas semiesferas, dois cilindros, ou uma semiesfera com um cilindro.

(i) Se $\mathrm{S}$ for formada por duas semiesferas $S e_{1}$ e $S e_{2}$ com raios $r_{1}$ e $r_{2}$ (por exemplo, $\left.r_{1} \geq r_{2}\right)$, que são mínimos relativos de (P), com áreas $A_{1}$ e $A_{2}$ e volumes $V_{1}$ e $V_{2}$, respectivamente, então a semiesfera $S e$ que encerra o mesmo volume $V=V_{1}+V_{2}$ tem área superficial A menor que a de $S e_{1}$ e $S e_{2}$ juntas.

De fato, como $V=V_{1}+V_{2}$, então o raio $\mathrm{r}$ da semiesfera $S e$ é tal que

$$
\frac{2}{3} \pi r^{3}=\frac{2}{3} \pi r_{1}^{3}+\frac{2}{3} \pi r_{2}^{3}, \quad \text { isto é, } \quad r=\left(r_{1}^{3}+r_{2}^{3}\right)^{1 / 3} \text {. }
$$

Além disto, a área A de Se é tal que:

$$
A^{3}=\left(2 \pi r^{2}\right)^{3}=(2 \pi)^{3}\left(r_{1}^{3}+r_{2}^{3}\right)^{2}=(2 \pi)^{3}\left(r_{1}^{6}+2 r_{1}^{3} r_{2}^{3}+r_{2}^{6}\right) .
$$

Como supomos $r_{2} \leq r_{1}$, então $2 r_{1}^{3} r_{2}^{3}=2 r_{1}^{3} r_{2} r_{2}^{2} \leq 2 r_{1}^{3} r_{1} r_{2}^{2}=2 r_{1}^{4} r_{2}^{2}$.

Logo, temos para (6.8) 


$$
A^{3} \leq(2 \pi)^{3}\left(r_{1}^{6}+2 r_{1}^{4} r_{2}^{2}+r_{2}^{6}\right)
$$

Além disto, $\left(r_{1}^{2}+r_{2}^{2}\right)^{3}=r_{1}^{6}+3 r_{1}^{4} r_{2}^{2}+3 r_{1}^{2} r_{2}^{4}+r_{2}^{6} \geq r_{1}^{6}+2 r_{1}^{4} r_{2}^{2}+r_{2}^{6}$.

Logo, temos para (6.9)

$$
A^{3} \leq(2 \pi)^{3}\left(r_{1}^{2}+r_{2}^{2}\right)^{3} \leq\left(2 \pi r_{1}^{2}+2 \pi r_{2}^{2}\right)^{3}=\left(A_{1}+A_{2}\right)^{3}
$$

Como $A, A_{1}, A_{2}$ são grandezas positivas, segue de (6.10) que $A \leq A_{1}+A_{2}$, e com isto a semiesfera $S e$ de raio r é o mínimo absoluto de $(\mathrm{P})$.

Se o raio r de $S e$ for tal que $r \geq d$, então temos que $\frac{2}{3} d<d \leq r$, isto é, $\frac{2}{3} d<r$. Logo, por (6.4) o cilindro $\mathrm{C}$ que encerra o mesmo volume $\mathrm{V}$ tem, no entanto, área superficial menor que a da semiesfera $S e$ de raio r e portanto, o cilindro é, neste caso, o mínimo absoluto de $(\mathrm{P})$.

(ii) Se $\mathrm{S}$ for formada por dois cilindros $C_{1}$ e $C_{2}$ com raios $\rho_{1}$ e $\rho_{2}$, que são mínimos relativos de $(\mathrm{P})$, com áreas superficiais $A_{1}$ e $A_{2}$ e volumes $V_{1}$ e $V_{2}$, respectivamente, então o cilindro $\mathrm{C}$ que encerra o mesmo volume $V=V_{1}+V_{2}$ tem área superficial menor que a de $C_{1}$ e $C_{2}$.

De fato, como $V=V_{1}+V_{2}$, então o raio $\rho$ de $\mathrm{C}$ é tal que

$$
\pi \rho^{2} d=\pi \rho_{1}^{2} d+\pi \rho_{2}^{2} d, \text { isto é, } \quad \rho=\left(\rho_{1}^{2}+\rho_{2}^{2}\right)^{1 / 2}
$$

Além disto, a área $\mathrm{A}$ de $\mathrm{C}$ é tal que:

$$
\begin{gathered}
A=2 \pi \rho d=2 \pi d\left(\rho_{1}^{2}+\rho_{2}^{2}\right)^{1 / 2} \leq 2 \pi d\left(\rho_{1}+\rho_{2}\right)=A_{1}+A_{2}, \text { visto que } \\
\left(\rho_{1}+\rho_{2}\right)^{2}=\rho_{1}^{2}+2 \rho_{1} \rho_{2}+\rho_{2}^{2} \geq \rho_{1}^{2}+\rho_{2}^{2}, \text { pois } \rho_{1}, \rho_{2}>0 . \text { Ou seja, } \\
A \leq A_{1}+A_{2}
\end{gathered}
$$

Como estamos supondo que os cilindros $C_{1}$ e $C_{2}$ são mínimos relativos de $(\mathrm{P})$, então vale por (6.6) que $\rho_{1}>\left(\frac{2}{3}\right)^{2} d$ e $\rho_{2}>\left(\frac{2}{3}\right)^{2} d$. Com isto, a semiesfera $S e$ de raio r, que encerra o mesmo volume $V=V_{1}+V_{2}$, tem área superficial maior que a de $\mathrm{C}$, pois por $(6.11)$ 
$\rho^{2}=\rho_{1}^{2}+\rho_{2}^{2}>\left(\frac{2}{3}\right)^{4} d^{2}+\left(\frac{2}{3}\right)^{4} d^{2}>\left(\frac{2}{3}\right)^{4} d^{2}$ e, consequentemente, $\rho>\left(\frac{2}{3}\right)^{2} d$. Agora, (6.6) justifica nossa afirmação.

Pela afirmação acima e por (6.12), concluímos que neste caso (ii) o cilindro C de raio $\rho$ é, de fato, o mínimo absoluto de $(\mathrm{P})$.

(iii) Se $\mathrm{S}$ for formada por uma semiesfera $S e_{1}$ de raio $r_{1}$ e um cilindro $C_{1}$ de raio $\rho_{1}$, que são mínimos relativos de $(\mathrm{P})$, com áreas superficiais $A_{1}$ e $A_{2}$ e volumes $V_{1}$ e $V_{2}$ respectivamente, então o cilindro $\mathrm{C}$ que encerra o mesmo volume $V=V_{1}+V_{2}$ tem área superficial A menor que a de $S e_{1}$ e $C_{1}$ juntas.

De fato, como $V=V_{1}+V_{2}$, então o raio $\rho$ de $\mathrm{C}$ é tal que

$$
\pi \rho^{2} d=\frac{2}{3} \pi r_{1}^{3}+\pi \rho_{1}^{2} d, \quad \text { isto é, } \quad \rho=\left(\frac{\frac{2}{3} r_{1}^{3}+\rho_{1}^{2} d}{d}\right)^{1 / 2} \text {. }
$$

Observamos que $\rho_{1}>\left(\frac{2}{3}\right)^{2} d$, pois, caso contrário, por (6.6) existiria uma semiesfera $S e_{2}$ de raio $r_{2}$ com $A\left(r_{2}\right)<A\left(\rho_{1}\right)$ e portanto, o cilindro $C_{1}$ não seria um mínimo relativo de $(\mathrm{P})$, o que contraria nossa hipótese. Além disto, vale $r_{1}<d$, para que a semiesfera $S e_{1}$ esteja entre os planos $P_{1}$ e $P_{2}$. Logo, a área superficial $\mathrm{A}$ de $\mathrm{C}$ é tal que

$$
\begin{aligned}
\left(A\left(r_{1}\right)+A\left(\rho_{1}\right)\right)^{2} & =\left(2 \pi r_{1}^{2}+2 \pi \rho_{1} d\right)^{2} \geq(2 \pi)^{2}\left(\rho_{1}^{2} d^{2}+2 r_{1}^{2}\left(\frac{2}{3}\right)^{2} d d\right) \geq \\
& \geq(2 \pi)^{2}\left(\rho_{1}^{2} d^{2}+\frac{2}{3} r_{1}^{2} r_{1} d\right),
\end{aligned}
$$

visto que $2\left(\frac{2}{3}\right)^{2}=\frac{4}{3} \frac{2}{3}>\frac{2}{3}$ e $d>r_{1}$. Logo, por (6.13) temos que

$$
\begin{aligned}
\left(A\left(r_{1}\right)+A\left(\rho_{1}\right)\right)^{2} & \geq(2 \pi)^{2}\left(\rho_{1}^{2} d^{2}+\frac{2}{3} r_{1}^{3} d\right)=\left(2 \pi \sqrt{\rho_{1}^{2} d^{2}+\frac{2}{3} r_{1}^{3} d}\right)^{2}= \\
& =\left(2 \pi d \sqrt{\frac{\rho_{1}^{2} d+\frac{2}{3} r_{1}^{3}}{d}}\right)^{2}=(2 \pi d \rho)^{2}=(A(\rho))^{2}
\end{aligned}
$$

ou seja,

$$
A(\rho) \leq A\left(r_{1}\right)+A\left(\rho_{1}\right)
$$

Conforme já mostramos, vale que $\rho_{1}>\left(\frac{2}{3}\right)^{2} d$. Por $(6.13)$, segue que

$\rho^{2}=\frac{\rho_{1}^{2} d+\frac{2}{3} r^{3}}{d}>\rho_{1}^{2}>\left(\frac{2}{3}\right)^{4} d^{2}$, ou seja, $\rho>\left(\frac{2}{3}\right)^{2} d$. Por (6.6), temos então que a semiesfera $S e$ de raio r que encerra o mesmo volume $V=V_{1}+V_{2}$ tem área superficial maior que a do cilindro $\mathrm{C}$ de raio $\rho$. 
Pela observação acima e por (6.14), concluímos que neste caso (iii) o cilindro C de raio $\rho$ é, de fato, o mínimo absoluto do problema $(\mathrm{P})$.

Continuando com os passos de indução de forma análoga ao que foi feito acima, concluímos que se a superfície $\mathrm{S}$ for um mínimo absoluto do problema variacional (P), então $\mathrm{S}$ deve ser conexa.

Com isto, resolvemos o problema (P) que foi objeto de estudo nesta dissertação. 


\section{Referências Bibliográficas}

[1] A. D. Alexandrov, Uniqueness theorems for surfaces in the large v, AMS Transl, 21 (1962).

[2] M. Athanassenas, Ein variationsproblem für flächen konstanter mittlerer krümmung mit freiem rand, master's thesis, Diplomarbeit, Bonn, 1985.

[3] _ A variational problem for constant mean curvature surfaces with free boundary, J. Reine Angew Math., 377 (1986), pp. 97-107.

[4] J. Barbosa AND M. Do Carmo, Stability of hypersurfaces with constant mean curvature, Mathematical Z, 185 (1984), pp. 339-353.

[5] J. BARBOSA AND R. EARP, Geometric methods and nonlinear analysis in hyperbolic space, in X escola de Geometria Diferencial - UFMG, 1998.

[6] O. BolzA, Lectures on the calculus of variations, Dover Publications.

[7] P. BÉRARD, Analysis on Riemannian manifolds and geometric applications-an introduction, no. 42 in Monografias de matemática.

[8] M. Carmo, Diferential Geometry of curves and surfaces, Prentice- Hall, New Jersey, 1976.

[9] —, Geometria Riemanniana, 2, Projeto Euclides IMPA - R.J, 1988.

[10] R. Courant and D. Hilbert, Methods of mathematical physics, vol. 1, Wiley Interscience, New York, 1953. 
[11] C. Delaunay, Sur la surface de révolution dont la courbure moyenne est constante, Journal de mathématiques pures et appliquées, 6 (1841), pp. 309 - 320.

[12] L. Elgoltz, Ecuaciones diferenciales y calculo variacional.

[13] D. Gilbarg And N. Trudinger, Elliptic partial differential equations of second order, Springer-Verlag, Berlin-Heidelberg-New York - Tokio, second ed., 1983.

[14] A. Gray, Modern Differential Geometry of curves and surfaces, C.R.C. Press. Inc, 1993.

[15] H. Hopf, Differential Geometry in the large, Lectures notes in Mathematics, no. 1000 .

[16] M. KoISO, Deformation and stability of surfaces with constant mean curvature, preprint.

[17] K. LEÃO, O princípio da tangência e aplicações, master's thesis, IMPA-R.J, 1983.

[18] E. LimA, Análise Real, vol. 1 of Coleção Matemática Universitária, CNPq IMPA R.J, 1989.

[19] J. Oprea, Differential Geometry and its applications, Prentice-Hall, Upper Saddle River, New Jersey.

[20] R. Pedrosa AND M. Ritoré, Isoperimetric domains in the riemannian product of a circle with a simply connected space form and applications to free boundary problems, preprint.

[21] E. Sallum, Cálculo Diferencial Geométrico no $\mathbb{R}^{n}$, IME-USP, 1999.

[22] T. VogeL, Stability of a drop trapped between two parallel planes, SIAM Journal Applied Mathemathics, 47 (1987). 